\title{
Bidirectional Decision Procedures for the Intuitionistic Propositional Modal Logic IS4
}

\author{
Samuli Heilala and Brigitte Pientka \\ School of Computer Science, McGill University, Montreal, Canada \\ \{sheila1, bpientka\}@cs.mcgill.ca
}

Technical Report SOCS-TR-2007.2, May 2007

\begin{abstract}
We present a multi-context focused sequent calculus whose derivations are in bijective correspondence with normal natural deductions in the propositional fragment of the intuitionistic modal logic IS4. This calculus, suitable for the enumeration of normal proofs, is the starting point for the development of a sequent calculus-based bidirectional decision procedure for propositional IS4. In this system, relevant derived inference rules are constructed in a forward direction prior to proof search, while derivations constructed using these derived rules are searched over in a backward direction. We also present a variant which searches directly over normal natural deductions. Experimental results show that on most problems, the bidirectional prover is competitive with both conventional backward provers using loop-detection and inverse method provers, significantly outperforming them in a number of cases.
\end{abstract}

\section{Introduction}

Intuitionistic modal logics are constructive logics incorporating operators of necessity $(\square)$ and possibility $(\diamond)$. Fitch [8], Prawitz [17], Satre [19], and more recently Simpson [20], Bierman and de Paiva [2], and Pfenning and Davies [16] have investigated a broad range of proof-theoretical properties of various logics of this kind. Recently, such logics have also found applications in hardware verification [7] and proposed type systems for staged computation [4] and distributed computing [14]. A logic frequently used in these settings is either the intuitionistic variant of the classical modal logic S4, which we will call IS4, or a logic that can be expressed through IS4, such as Fairtlough and Mendler's lax logic [7] (see for instance [16] for the relationship between IS4 and lax logic).

In this light, it is surprising that proof search in IS4 has not received more attention. Howe has investigated proof enumeration and theorem proving in lax logic [13] and, coming closer to our work, has presented a backward decision procedure for the fragment of propositional IS4 without the possibility modality [12]. His system performs loop-detection using a history mechanism, but is encumbered by a large number of rules and related provisos (21 axioms and inference rules). It would only grow with the addition of the possibility modality, which would also require a different loop-detection mechanism. 
Our contributions begin with a sequent calculus for propositional IS4 suitable for the enumeration of normal proofs. This forms the basis for the development of a sequent calculus-based bidirectional IS4 decision procedure, in which derived inference rules relevant to the query are constructed in a forward direction prior to proof search, while derivations constructed using these derived rules are searched over in a backward direction. We also demonstrate that this approach corresponds very closely to an elegant bidirectional decision procedure that searches directly over normal natural deductions. The key to our theoretical justification of both of these decision procedures is a refinement of the well-known subformula property, which we use to restrict nondeterminism in focused proof search in the presence of multiple contexts. To evaluate our approach empirically, we have put together a set of 50 benchmark formulas for IS4. Experimental results show that on most problems, the bidirectional prover is competitive with both conventional backward provers using loop-detection and inverse method provers, significantly outperforming them in a number of cases. Although we concentrate on propositional IS4 in this paper, we believe that the techniques presented are general enough to find applications in other constructive logics, such as the contextual modal logic of Nanevski, Pfenning, and Pientka [15]. This paper is an extended version of [11].

In Sect. 2 we summarize the relevant background and introduce our core natural deduction formalism, while Sect. 3 presents a corresponding Hilbertstyle axiomatization. In Sect. 4 we restrict our natural deduction system to allow only normal natural deductions, and in Sect. 5 we give the corresponding sequent calculus formulation that serves as the basis for our proof search strategies. A more refined sequent calculus for forward proof search is developed in Sect. 6, while Sects. 7 and 8 describe our bidirectional decision procedure in sequent calculus and natural deduction settings, respectively. Experimental results are given in Sect. 9, and Sect. 10 concludes with related and future work.

\section{Natural Deduction}

Formulas in the propositional fragment of IS4 are given by the grammar

$$
A \quad::=\quad P|\perp| A \supset A|A \wedge A| A \vee A|\square A| \diamond A
$$

where $P$ is taken from a countable set of atomic propositional constants and negation and truth are defined notationally in the usual way:

$$
\neg A \stackrel{\text { def }}{=} A \supset \perp \quad T \stackrel{\text { def }}{=} \neg \perp
$$

Operator precedences are, from highest to lowest, $\square, \diamond, \wedge, \vee, \supset$, while all binary operators are considered right-associative. Our starting point is a multi-context natural deduction formulation for IS4 similar to ones proposed by Pfenning and Davies [16] and Bierman and de Paiva [2], featuring hypothetical judgements of the form $\Delta ; \Gamma \vdash A$. Such a judgement asserts the provability of a formula $A$ under hypotheses $\Delta$ and $\Gamma$, where $\Gamma=A_{1}, \ldots, A_{n}$ is a context of true hypotheses 


$$
\begin{aligned}
& \overline{\Delta ; \Gamma_{1}, A, \Gamma_{2} \vdash A} \operatorname{hyp}_{1} \quad \overline{\Delta_{1}, A, \Delta_{2} ; \Gamma \vdash A} \operatorname{hyp}_{2} \frac{\Delta ; \Gamma \vdash \perp}{\Delta ; \Gamma \vdash A} \perp \mathrm{E} \\
& \frac{\Delta ; \Gamma, A_{1} \vdash A_{2}}{\Delta ; \Gamma \vdash A_{1} \supset A_{2}} \supset \mathrm{I} \quad \frac{\Delta ; \Gamma \vdash A_{1} \supset A_{2} \quad \Delta ; \Gamma \vdash A_{1}}{\Delta ; \Gamma \vdash A_{2}} \supset \mathrm{E} \\
& \frac{\Delta ; \Gamma \vdash A_{1} \quad \Delta ; \Gamma \vdash A_{2}}{\Delta ; \Gamma \vdash A_{1} \wedge A_{2}} \wedge \mathrm{I} \frac{\Delta ; \Gamma \vdash A_{1} \wedge A_{2}}{\Delta ; \Gamma \vdash A_{j}} \wedge \mathrm{E}_{j} \\
& \frac{\Delta ; \Gamma \vdash A_{j}}{\Delta ; \Gamma \vdash A_{1} \vee A_{2}} \vee \mathrm{I}_{j} \quad \frac{\Delta ; \Gamma \vdash A_{1} \vee A_{2} \quad \Delta ; \Gamma, A_{1} \vdash C \quad \Delta ; \Gamma, A_{2} \vdash C}{\Delta ; \Gamma \vdash C} \vee \mathrm{E} \\
& \frac{\Delta ; \cdot \vdash A}{\Delta ; \Gamma \vdash \square A} \square \quad \square \quad \frac{\Delta ; \Gamma \vdash \square A \quad \Delta, A ; \Gamma \vdash C}{\Delta ; \Gamma \vdash C} \square \mathrm{E} \\
& \frac{\Delta ; \Gamma \vdash A}{\Delta ; \Gamma \vdash \diamond A} \diamond \mathrm{I} \frac{\Delta ; \Gamma \vdash \diamond A \quad \Delta ; A \vdash \diamond C}{\Delta ; \Gamma \vdash \diamond C} \diamond \mathrm{E} \\
& j \in\{1,2\}
\end{aligned}
$$

Fig. 1. $\mathrm{NJ}_{\text {IS4 }}$

and $\Delta=B_{1}, \ldots, B_{m}$ is a modal context of valid hypotheses. Valid hypotheses are hypotheses whose truth does not depend on the truth of other formulas, that is, hypotheses that are in some sense "always" or necessarily true. We use - to denote the empty context. Our base system differs from Pfenning and Davies' natural deduction formulation for IS4 [16] in that we make no judgemental distinction between truth and possibility. Instead, we internalize the possibility judgement using the $\diamond$ operator. Interestingly, this gives rise to a system that is essentially identical to Bierman and de Paiva's multi-context natural deduction formulation for IS4 [2]. This base calculus, which we will call $\mathbf{N J}_{\mathbf{I S} 4}$, is shown in Fig. 1.

Although the contexts of this system are formally ordered lists, we can afford to be flexible with them, as $\mathbf{N} \mathbf{J}_{\text {IS4 }}$ has the usual structural properties of weakening, contraction, and exchange for both contexts. We formalize some of these properties below. In the remainder, we will not always formally state structural properties that are not required for the proofs of the main results. For convenience, we will generally think of contexts in $\mathbf{N J}_{\mathbf{I S} 4}$ as multisets, and will write $A \in \Gamma$ to denote that $\Gamma$ has the form $\Gamma_{1}, A, \Gamma_{2}$ for some (possibly empty) $\Gamma_{1}$ and $\Gamma_{2}$.

\section{Lemma 1 (Weakening for $\mathrm{NJ}_{\mathrm{IS} 4}$ ).}

If $\Delta ; \stackrel{\mathcal{D}}{\mathcal{D}} \Gamma_{1}, \Gamma_{2} \vdash C$, then $\Delta ; \Gamma_{1}, A, \Gamma_{2} \vdash C$.

Proof. By induction on the structure of $\mathcal{D} .^{1}$

\footnotetext{
${ }^{1}$ Whenever a proof is by a very simple induction, we will omit the cases.
} 
Lemma 2 (Modal Weakening for $\mathrm{NJ}_{\mathrm{IS} 4}$ ).

If $\Delta_{1}, \Delta_{2}^{\mathcal{D}} ; \Gamma \vdash C$, then $\Delta_{1}, A, \Delta_{2} ; \Gamma \vdash C$.

Proof. By induction on the structure of $\mathcal{D}$.

Lemma 3 (Exchange for $\mathrm{NJ}_{\mathrm{IS} 4}$ ).

If $\Delta ; \Gamma_{1}, A_{1}, \Gamma_{2}, A_{2}, \Gamma_{3} \vdash C$, then $\Delta ; \Gamma_{1}, A_{2}, \Gamma_{2}, A_{1}, \Gamma_{3} \vdash C$.

Proof. By induction on the structure of $\mathcal{D}$.

Lemma 4 (Modal exchange for $\mathrm{NJ}_{\mathrm{IS} 4}$ ).

If $\Delta_{1}, A_{1}, \Delta_{2}, A_{2}, \Delta_{3} ; \Gamma \vdash C$, then $\Delta_{1}, A_{2}, \Delta_{2}, A_{1}, \Delta_{3} ; \Gamma \vdash C$.

Proof. By induction on the structure of $\mathcal{D}$.

The system $\mathbf{N} \mathbf{J}_{\text {IS4 }}$ also has the following important substitution properties. They encapsulate the intuitive notion of replacing uses of a hypothesis by separate derivations of that hypothesis.

Lemma 5 (Substitution property for $\mathrm{NJ}_{\mathrm{IS} 4}$ ). If $\Delta ; \Gamma_{1}, A, \Gamma_{2} \vdash C$ and $\Delta ; \Gamma_{1}, \Gamma_{2} \vdash A$, then $\Delta ; \Gamma_{1}, \Gamma_{2} \vdash C$.

Proof. By induction on the structure of $\mathcal{D}$, using Lemmas 1 and 2 where needed.

Lemma 6 (Modal substitution property for $\mathrm{NJ}_{\text {IS4 }}$ ).

If $\Delta_{1}, A, \stackrel{\mathcal{D}}{\Delta_{2} ; \Gamma \vdash C}$ and $\Delta_{1}, \Delta_{2} ; \vdash \cdot$, then $\Delta_{1}, \Delta_{2} ; \Gamma \vdash C$.

Proof. By induction on the structure of $\mathcal{D}$, using Lemmas 1 and 2 where needed.

The inference rules of $\mathbf{N J}_{\text {IS4 }}$ are largely standard, but to glean some intuition about the modal rules and the two contexts, it is useful to think of the modalities as quantifying truth over worlds in some universe, with some reachability relation defined on the worlds. To say that $\square A$ is true is to say that $A$ is true in all worlds reachable from the current one, while to say that $\diamond A$ is true is to say that $A$ is true in some world reachable from the current one. The current world represents the environment in which the provability of the succedent is to be established. Under this interpretation, the hypotheses in the modal context can be used in all reachable worlds, while those in the regular context can only be used in the current world. This notion is formalized by the following results, which highlight the relationship between global validity in the form of the modal context and the local internalization of validity in the form of the necessity operator.

Theorem 1 (Globalizing necessity in $\mathrm{NJ}_{\mathrm{IS} 4}$ ). $\mathcal{D}$

If $\Delta_{1}, \Delta_{2} ; \Gamma_{1}, \square A, \Gamma_{2} \vdash C$, then $\Delta_{1}, A, \Delta_{2} ; \Gamma_{1}, \Gamma_{2} \vdash C$. 
Proof.

(1) $\Delta_{1}, A, \Delta_{2} ; \Gamma_{1}, \square A, \Gamma_{2} \vdash C$

Lemma $2(\mathcal{D})$

(2) $\Delta_{1}, A, \Delta_{2} ; \cdot \vdash A$

hyp $_{2}$

(3) $\Delta_{1}, A, \Delta_{2} ; \Gamma_{1}, \Gamma_{2} \vdash \square A$

(4) $\Delta_{1}, A, \Delta_{2} ; \Gamma_{1}, \Gamma_{2} \vdash C$

Lemma $5(1,3)$

Theorem 2 (Localizing validity in $\mathrm{NJ}_{\mathrm{IS} 4}$ ).

If $\Delta_{1}, A, \Delta_{2} ; \Gamma_{1}, \Gamma_{2} \vdash C$, then $\Delta_{1}, \Delta_{2} ; \Gamma_{1}, \square A, \Gamma_{2} \vdash C$.

Proof.

(1) $\Delta_{1}, A, \Delta_{2} ; \Gamma_{1}, \square A, \Gamma_{2} \vdash C$

Lemma $1(\mathcal{D})$

(2) $\Delta_{1}, \Delta_{2}, A ; \Gamma_{1}, \square A, \Gamma_{2} \vdash C$

Lemma 4 (1)

(3) $\Delta_{1}, \Delta_{2} ; \Gamma_{1}, \square A, \Gamma_{2} \vdash \square A$

hyp $_{1}$

(4) $\Delta_{1}, \Delta_{2} ; \Gamma_{1}, \square A, \Gamma_{2} \vdash C$

$\square \mathrm{E}(3,2)$

Theorem 2 suggests that the contextual separation of truth and validity is not strictly necessary, and that we can obtain an embedding of $\mathbf{N} \mathbf{J}_{\mathbf{I S} \mathbf{4}}$ into a single-context system simply by providing every valid hypothesis with a $\square$ operator and merging the two contexts. To obtain a single-context system of natural deduction for IS4, however, those inference rules that depend on the separation of the modal and regular contexts (such as $\square \mathrm{I}, \square \mathrm{E}$, and $\diamond \mathrm{E}$ ) need to be modified. The details of single-context natural deduction systems for IS4 have been explored in more detail by Bierman and de Paiva [2].

To lend support to our claim that $\mathbf{N J}_{\mathbf{I S} \mathbf{4}}$ is indeed a formalism for a constructive variant of the classical modal logic $\mathbf{S 4}$, we will next present a Hilbert-style axiomatization of IS4 and show that $\mathbf{N J}_{\mathbf{I S} 4}$ is equivalent to it.

\section{Axiomatization}

While several proposals for Hilbert-style axiomatic formulations of constructive versions of $\mathbf{S} \mathbf{4}$ exist in the literature, we will use a standard system motivated by applications discussed by Alechina et al. [1] and Pfenning and Davies [16]. In it, a standard axiomatization of intuitionistic propositional logic (see for instance $[21])$ is augmented by the six modal axioms

$$
\begin{aligned}
K_{\square}: \square\left(A_{1} \supset A_{2}\right) \supset \square A_{1} \supset \square A_{2} & & K_{\diamond}: \square\left(A_{1} \supset A_{2}\right) \supset \diamond A_{1} \supset \diamond A_{2} \\
T_{\square}: \square A \supset A & & T_{\diamond}: A \supset \diamond A \\
4_{\square}: \square A \supset \square \square A & & 4 \diamond: \diamond \diamond A \supset \diamond A
\end{aligned}
$$

and the necessitation rule "If $A$ is a theorem, then $\square A$ is a theorem." The complete axiomatization, which we will call $\mathbf{A}_{\mathbf{I S} 4}$, is shown in Fig. 2. For consistency with $\mathbf{N J}_{\mathbf{I S} 4}$, we again consider contexts to be ordered lists, but an interpretation of contexts as sets yields the same results. Note that we show general weakening as an explicit rule $w$, while an alternative approach, followed by Bierman and de 


\section{Axioms:}

$$
\begin{array}{rlrl}
\supset_{1} & : A_{1} \supset A_{2} \supset A_{1} & & \supset_{2}:\left(A_{1} \supset A_{2} \supset A_{3}\right) \supset\left(A_{1} \supset A_{2}\right) \supset A_{1} \supset A_{3} \\
\wedge_{1}: A_{1} \supset A_{2} \supset A_{1} \wedge A_{2} & \vee_{1}:\left(A_{1} \supset A_{3}\right) \supset\left(A_{2} \supset A_{3}\right) \supset A_{1} \vee A_{2} \supset A_{3} \\
\wedge_{2}: A_{1} \wedge A_{2} \supset A_{1} & \vee_{2}: A_{1} \supset A_{1} \vee A_{2} \\
\wedge_{3}: A_{1} \wedge A_{2} \supset A_{2} & \vee_{3}: A_{2} \supset A_{1} \vee A_{2} \\
\perp: \perp \supset A & & \\
\perp K_{\square}: \square\left(A_{1} \supset A_{2}\right) \supset \square A_{1} \supset \square A_{2} & K_{\diamond}: \square\left(A_{1} \supset A_{2}\right) \supset \diamond A_{1} \supset \diamond A_{2} \\
T_{\square}: \square A \supset A & & T_{\diamond}: A \supset \diamond A \\
4_{\square}: \square A \supset \square \square A & & 4 \diamond: \diamond \diamond A \supset \diamond A
\end{array}
$$

Rules:

$$
\begin{gathered}
\overline{A \vdash_{\mathbf{A}} A} \text { ass } \frac{\vdash_{\mathbf{A} A} \text { ax }(A \text { is an axiom })}{\frac{\Gamma_{1}, \Gamma_{2} \vdash_{\mathbf{A}} C}{\Gamma_{1}, A, \Gamma_{2} \vdash_{\mathbf{A}} C}} \text { w } \\
\frac{\Gamma \vdash_{\mathbf{A}} A_{1} \supset A_{2} \quad \Gamma \vdash_{\mathbf{A}} A_{1}}{\Gamma \vdash_{\mathbf{A}} A_{2}} \mathrm{mp} \quad \frac{\cdot \vdash_{\mathbf{A}} A}{\cdot \vdash_{\mathbf{A}} \square A} \text { nec }
\end{gathered}
$$

Fig. 2. A IS4

Paiva [2], is to incorporate weakening locally into the assumption, axiom, and necessitation rules, and to prove its general form to be admissible. We will use $\mathrm{w}^{*}$ to denote zero or more applications of the weakening rule $\mathrm{w}$, and ass* and ax* to denote applications of the assumption rule ass and the axiom rule ax, respectively, followed by zero or more applications of the weakening rule w.

The following common intermediate results hold for our axiomatization.

\section{Lemma 7 (Reflexivity of $\supset$ for $\mathrm{A}_{\mathrm{IS} 4}$ ).}

$$
\Gamma \vdash_{A} A \supset A \text {. }
$$

Proof.

(1) $\Gamma \vdash_{\mathbf{A}}(A \supset(A \supset A) \supset A) \supset(A \supset A \supset A) \supset A \supset A \quad \operatorname{ax}^{*}\left(\supset_{2}\right)$

(2) $\Gamma \vdash_{\mathbf{A}} A \supset(A \supset A) \supset A$

(3) $\Gamma \vdash_{\mathbf{A}}(A \supset A \supset A) \supset A \supset A$

$\mathrm{mp}(1,2)$

(4) $\Gamma \vdash_{\mathbf{A}} A \supset A \supset A$

$\mathrm{ax}^{*}\left(\supset_{1}\right)$

(5) $\Gamma \vdash_{\mathbf{A}} A \supset A$

$\mathrm{mp}(3,4)$

Lemma 8 (Deduction theorem for $\mathrm{A}_{\mathrm{IS} 4}$ ).

If $\Gamma_{1}, A_{1}, \stackrel{\mathcal{D}}{\Gamma_{2}} \vdash_{\boldsymbol{A}} A_{2}$, then $\Gamma_{1}, \Gamma_{2} \vdash{ }_{\boldsymbol{A}} A_{1} \supset A_{2}$.

Proof. By induction on the structure of $\mathcal{D}$.

\section{Case:}

$$
\mathcal{D}={\overline{A \vdash_{\mathbf{A}} A}}^{\text {ass }}
$$

$$
(1) \cdot \vdash_{\mathbf{A}} A \supset A
$$




\section{Case:}

$$
\mathcal{D}=\frac{\mathcal{D}_{1}, \Gamma_{2} \vdash_{\mathbf{A}} A_{2}}{\Gamma_{1}, A_{1}, \Gamma_{2} \vdash_{\mathbf{A}} A_{2}} \mathrm{w}
$$

(1) $\Gamma_{1}, \Gamma_{2} \vdash_{\mathbf{A}} A_{2} \supset A_{1} \supset A_{2}$

$\mathrm{ax}^{*}\left(\supset_{1}\right)$

(2) $\Gamma_{1}, \Gamma_{2} \vdash_{\mathbf{A}} A_{1} \supset A_{2}$

Case:

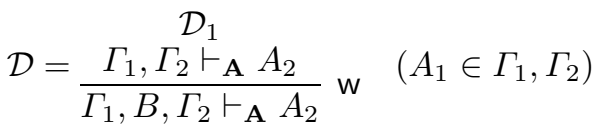

(1) $\Gamma_{1}^{\prime}, \Gamma_{2}^{\prime} \vdash_{\mathbf{A}} A_{1} \supset A_{2}$

i.h. $\left(\mathcal{D}_{1}\right)$

(2) $\Gamma_{1}^{\prime}, B, \Gamma_{2}^{\prime} \vdash_{\mathbf{A}} A_{1} \supset A_{2}$

Case:

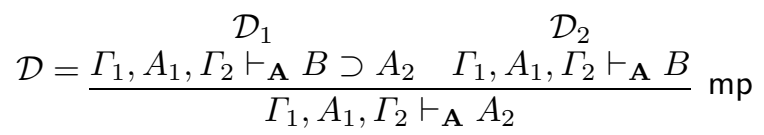

(1) $\Gamma_{1}, \Gamma_{2} \vdash_{\mathbf{A}}\left(A_{1} \supset B \supset A_{2}\right) \supset\left(A_{1} \supset B\right) \supset A_{1} \supset A_{2}$

$\operatorname{ax}^{*}\left(\supset_{2}\right)$

(2) $\Gamma_{1}, \Gamma_{2} \vdash_{\mathbf{A}} A_{1} \supset B \supset A_{2}$

i.h. $\left(\mathcal{D}_{1}\right)$

(3) $\Gamma_{1}, \Gamma_{2} \vdash_{\mathbf{A}}\left(A_{1} \supset B\right) \supset A_{1} \supset A_{2}$

$\mathrm{mp}(1,2)$

(4) $\Gamma_{1}, \Gamma_{2} \vdash_{\mathbf{A}} A_{1} \supset B$

i.h. $\left(\mathcal{D}_{2}\right)$

(5) $\Gamma_{1}, \Gamma_{2} \vdash_{\mathbf{A}} A_{1} \supset A_{2}$

mp $(3,4)$

Lemma 9 (Transitivity of $\supset$ for $\mathrm{A}_{\text {IS4 }}$ ).

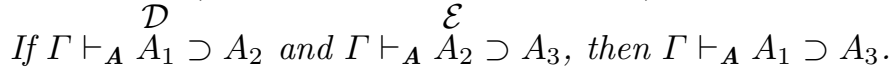

Proof.

(1) $\Gamma, A_{1} \vdash_{\mathbf{A}} A_{2} \supset A_{3}$

(2) $\Gamma \vdash_{\mathbf{A}} A_{1} \supset A_{2} \supset A_{3}$

(3) $\Gamma \vdash_{\mathbf{A}}\left(A_{1} \supset A_{2} \supset A_{3}\right) \supset\left(A_{1} \supset A_{2}\right) \supset A_{1} \supset A_{3}$

(4) $\Gamma \vdash_{\mathbf{A}}\left(A_{1} \supset A_{2}\right) \supset A_{1} \supset A_{3}$

(5) $\Gamma \vdash_{\mathbf{A}} A_{1} \supset A_{3}$

Lemma 10 (Generalized necessitation for $\mathbf{A}_{\mathrm{IS} 4}$ ).

If $\square A_{1}, \ldots, \stackrel{\mathcal{D}}{\square} A_{n} \vdash_{\boldsymbol{A}} C$, then $\square A_{1}, \ldots, \square A_{n} \vdash_{\boldsymbol{A}} \square C$.

Proof. By induction on $n$.

Case: $n=0$.

$$
(1) \cdot \vdash_{\mathbf{A}} \square C
$$

$\operatorname{nec}(\mathcal{D})$ 
Case: $n>0$.

(1) $\square A_{1}, \ldots, \square A_{n-1} \vdash_{\mathbf{A}} \square A_{n} \supset C$

Lemma $8(\mathcal{D})$

(2) $\square A_{1}, \ldots, \square A_{n-1} \vdash_{\mathbf{A}} \square\left(\square A_{n} \supset C\right)$

(3) $\square A_{1}, \ldots, \square A_{n-1} \vdash_{\mathbf{A}} \square\left(\square A_{n} \supset C\right) \supset \square \square A_{n} \supset \square C$

i.h. (1)

(4) $\square A_{1}, \ldots, \square A_{n-1} \vdash_{\mathbf{A}} \square \square A_{n} \supset \square C$

$\mathrm{ax}^{*}\left(K_{\square}\right)$

$\mathrm{mp}(3,2)$

(5) $\square A_{1}, \ldots, \square A_{n-1} \vdash_{\mathbf{A}} \square A_{n} \supset \square \square A_{n}$

$\mathrm{ax}^{*}(4 \square)$

(6) $\square A_{1}, \ldots, \square A_{n-1} \vdash_{\mathbf{A}} \square A_{n} \supset \square C$

(7) $\square A_{1}, \ldots, \square A_{n-1}, \square A_{n} \vdash_{\mathbf{A}} \square A_{n} \supset \square C$

Lemma $9(5,4)$

(8) $\square A_{1}, \ldots, \square A_{n-1}, \square A_{n} \vdash_{\mathbf{A}} \square A_{n}$

w (6)

(9) $\square A_{1}, \ldots, \square A_{n-1}, \square A_{n} \vdash_{\mathbf{A}} \square C$

$\mathrm{mp}(7,8)$

Armed with these intermediate results, we can now prove the equivalence of $\mathbf{N J}_{\mathbf{I S} 4}$ and $\mathbf{A}_{\mathbf{I S} 4}$. As a shorthand, if $\Delta=A_{1}, \ldots, A_{n}$, then we will write $\square \Delta$ to denote $\square A_{1}, \ldots, \square A_{n}$.

Theorem 3 (Soundness of $\mathrm{NJ}_{\mathrm{IS} 4}$ with respect to $\mathrm{A}_{\mathrm{IS} 4}$ ).

If $\Delta ; \stackrel{\mathcal{D}}{\Gamma} \vdash A$, then $\square \Delta, \Gamma \vdash{ }_{\boldsymbol{A}} A$.

Proof. By induction on the structure of $\mathcal{D}$. We show the base cases and the cases involving modalities.

Case:

$$
\mathcal{D}=\overline{\Delta ; \Gamma_{1}, A, \Gamma_{2} \vdash A} \text { hyp }_{1}
$$

(1) $\square \Delta, \Gamma_{1}, A, \Gamma_{2} \vdash_{\mathbf{A}} A \quad$ ass*

Case:

$$
\mathcal{D}=\overline{\Delta_{1}, A, \Delta_{2} ; \Gamma \vdash A} \text { hyp }_{2}
$$

(1) $\square \Delta_{1}, \square A, \square \Delta_{2}, \Gamma \vdash_{\mathbf{A}} \square A \supset A$

(2) $\square \Delta_{1}, \square A, \square \Delta_{2}, \Gamma \vdash_{\mathbf{A}} \square A$

$\mathrm{ax}^{*}\left(T_{\square}\right)$

(3) $\square \Delta_{1}, \square A, \square \Delta_{2}, \Gamma \vdash_{\mathbf{A}} A$

$\mathrm{mp}(1,2)$

Case:

$$
\mathcal{D}=\frac{\mathcal{D}_{1} ; \cdot \vdash A}{\Delta ; \Gamma \vdash \square A} \square \mathrm{I}
$$

(1) $\square \Delta \vdash_{\mathbf{A}} A$

i.h. $\left(\mathcal{D}_{1}\right)$

(2) $\square \Delta \vdash_{\mathbf{A}} \square A$

Lemma 10 (1)

(3) $\square \Delta, \Gamma \vdash_{\mathbf{A}} \square A$

$\mathrm{w}^{*}(2)$

Case:

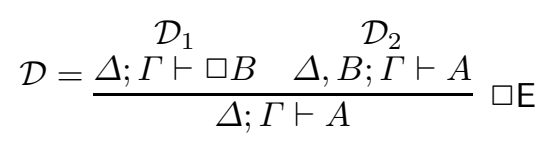

(1) $\square \Delta, \square B, \Gamma \vdash_{\mathbf{A}} A$

(2) $\square \Delta, \Gamma \vdash_{\mathbf{A}} \square B \supset A$

i.h. $\left(\mathcal{D}_{2}\right)$

(3) $\square \Delta, \Gamma \vdash_{\mathbf{A}} \square B$

Lemma 8 (1)

(4) $\square \Delta, \Gamma \vdash_{\mathbf{A}} A$

i.h. $\left(\mathcal{D}_{1}\right)$

mp $(2,3)$ 


\section{Case:}

$$
\mathcal{D}=\frac{\Delta ; \Gamma \vdash A}{\mathcal{D}_{1}} \underset{\Delta ; \Gamma \vdash \diamond A}{ } \diamond
$$
(1) $\square \Delta, \Gamma \vdash_{\mathbf{A}} A$
i.h. $\left(\mathcal{D}_{1}\right)$
(2) $\square \Delta, \Gamma \vdash_{\mathbf{A}} A \supset \diamond A$
$\mathrm{ax}^{*}\left(T_{\diamond}\right)$
(3) $\square \Delta, \Gamma \vdash_{\mathbf{A}} \diamond A$
$\mathrm{mp}(2,1)$

Case:

$$
\mathcal{D}=\frac{\stackrel{\mathcal{D}_{1}}{\Delta ; \Gamma \vdash \diamond B \quad} \stackrel{\mathcal{D}_{2}}{\Delta ; B \vdash \diamond A}}{\Delta ; \Gamma \vdash \diamond A} \diamond \mathrm{E}
$$

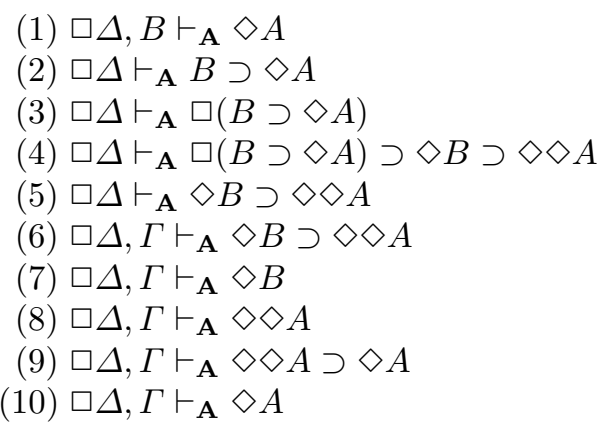

i.h. $\left(\mathcal{D}_{2}\right)$

Lemma 8 (1)

Lemma $10(2)$

$\operatorname{ax}^{*}\left(K_{\diamond}\right)$

$\mathrm{mp}(4,3)$

$\mathrm{w}^{*}(5)$

i.h. $\left(\mathcal{D}_{1}\right)$

$\mathrm{mp}(6,7)$

$\mathrm{ax}^{*}(4 \diamond)$

$\mathrm{mp}(9,8)$

Lemma 11 (Completeness of $\mathrm{NJ}_{\mathrm{IS} 4}$ with respect to the axioms of

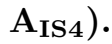

If $A$ is an axiom of $\mathbf{A}_{\mathbf{I S} 4}$, then $\cdot ; \cdot \vdash A$.

Proof. We show the cases involving modalities. The derivations are linearized to save space.

Case: $K_{\square}$
(1) $\cdot ; \square\left(A_{1} \supset A_{2}\right), \square A_{1} \vdash \square\left(A_{1} \supset A_{2}\right)$
(2) $A_{1} \supset A_{2} ; \square\left(A_{1} \supset A_{2}\right), \square A_{1} \vdash \square A_{1}$
(3) $A_{1} \supset A_{2}, A_{1} ; \cdot \vdash A_{1} \supset A_{2}$
(4) $A_{1} \supset A_{2}, A_{1} ; \cdot \vdash A_{1}$
(5) $A_{1} \supset A_{2}, A_{1} ; \cdot \vdash A_{2}$
(6) $A_{1} \supset A_{2}, A_{1} ; \square\left(A_{1} \supset A_{2}\right), \square A_{1} \vdash \square A_{2}$
(7) $A_{1} \supset A_{2} ; \square\left(A_{1} \supset A_{2}\right), \square A_{1} \vdash \square A_{2}$
(8) $\cdot$; $\square\left(A_{1} \supset A_{2}\right), \square A_{1} \vdash \square A_{2}$
(9) $; \square\left(A_{1} \supset A_{2}\right) \vdash \square A_{1} \supset \square A_{2}$
(10) $; \cdot \vdash \square\left(A_{1} \supset A_{2}\right) \supset \square A_{1} \supset \square A_{2}$
hyp $_{1}$ hyp $_{2}$ $\supset \mathrm{E}(3,4)$ 미 (5) $\square \mathrm{E}(2,6)$ $\square \mathrm{E}(1,7)$ つl (8) गI (9) hyp $_{1}$ hyp $_{2}$ 
Case: $K_{\diamond}$

(1) $\cdot ; \square\left(A_{1} \supset A_{2}\right), \diamond A_{1} \vdash \square\left(A_{1} \supset A_{2}\right)$

(2) $A_{1} \supset A_{2} ; \square\left(A_{1} \supset A_{2}\right), \diamond A_{1} \vdash \diamond A_{1}$

(3) $A_{1} \supset A_{2} ; A_{1} \vdash A_{1} \supset A_{2}$

(4) $A_{1} \supset A_{2} ; A_{1} \vdash A_{1}$

(5) $A_{1} \supset A_{2} ; A_{1} \vdash A_{2}$

(6) $A_{1} \supset A_{2} ; A_{1} \vdash \diamond A_{2}$

(7) $A_{1} \supset A_{2} ; \square\left(A_{1} \supset A_{2}\right), \diamond A_{1} \vdash \diamond A_{2}$

(8) $\cdot$; $\square\left(A_{1} \supset A_{2}\right), \diamond A_{1} \vdash \diamond A_{2}$

(9) $; \square\left(A_{1} \supset A_{2}\right) \vdash \diamond A_{1} \supset \diamond A_{2}$

$(10) \cdot ; \cdot \vdash\left(A_{1} \supset A_{2}\right) \supset \diamond A_{1} \supset \diamond A_{2}$

Case: $T_{\square}$

(1) $\cdot ; \square A \vdash \square A$

(2) $A$; $\square A \vdash A$

(3) $\cdot ; \square A \vdash A$

(4) $\cdot ; \cdot \vdash \square A \supset A$

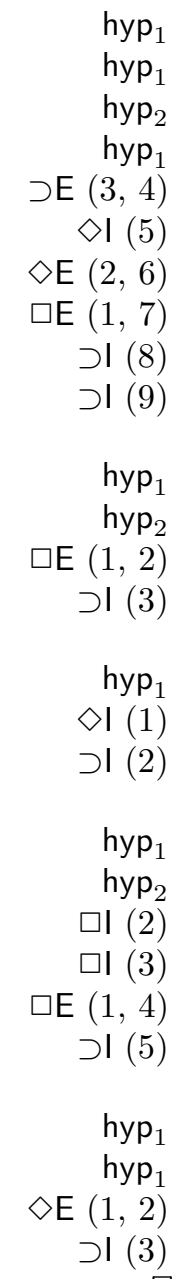

$>$ I (1)

hyp

$(1,2)$

Theorem 4 (Completeness of $\mathrm{NJ}_{\mathrm{IS} 4}$ with respect to $\left.\mathrm{A}_{\mathrm{IS} 4}\right)$. If $\Gamma \stackrel{\mathcal{D}}{\vdash}_{\boldsymbol{A}} A$, then $\cdot ; \Gamma \vdash A$.

Proof. By induction on the structure of $\mathcal{D}$.

Case:

(1) $\cdot ; A \vdash A$

(2) $; ; A \vdash \diamond A$

(3) $\cdot ; \cdot \vdash A \supset \diamond A$

Case: 4

(1) $\cdot ; \square A \vdash \square A$

(2) $A ; \cdot \vdash A$

(3) $A ; \cdot \vdash \square A$

(4) $A ; \square A \vdash \square \square A$

(5) $\cdot$; $\square A \vdash \square \square A$

(6) $\cdot ; \cdot \vdash \square A \supset \square \square A$

(1) $\cdot ; \diamond \diamond A \vdash \diamond \diamond A$

(2) $\cdot ; \diamond A \vdash \diamond A$

(3) $\cdot ; \diamond \diamond A \vdash \diamond A$

(4) $\cdot ; \cdot \vdash \diamond \diamond A \supset \diamond A$

hyp $_{1}$

\section{Case:}

$$
\mathcal{D}={\overline{A \vdash_{\mathbf{A}} A}}^{\text {ass }}
$$

(1) $\cdot ; A \vdash A$

$$
\mathcal{D}=\overline{\vdash_{\mathbf{A}} A} \text { ax }
$$

(1) $\cdot ; \cdot \vdash A$ 
Case:

$$
\mathcal{D}=\frac{\Gamma_{1}, \Gamma_{2} \vdash_{\mathbf{A}} A}{\Gamma_{1}, B, \Gamma_{2} \vdash_{\mathbf{A}} A} \mathrm{w}
$$

(1) $\cdot ; \Gamma_{1}, \Gamma_{2} \vdash A$

i.h. $\left(\mathcal{D}_{1}\right)$

Case:

(2) $\cdot ; \Gamma_{1}, B, \Gamma_{2} \vdash A$

Lemma 1 (1)

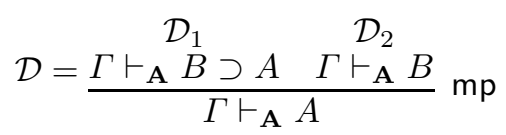

(1) $\cdot ; \Gamma \vdash B \supset A$

i.h. $\left(\mathcal{D}_{1}\right)$

(2) $\cdot ; \Gamma \vdash B$

i.h. $\left(\mathcal{D}_{2}\right)$

(3) $\cdot ; \Gamma \vdash A$

$\supset \mathrm{E}(1,2)$

Case:

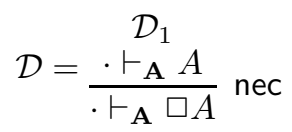

(1) $\cdot ; \cdot \vdash A$

i.h. $\left(\mathcal{D}_{1}\right)$

(2) $\cdot ; \cdot \vdash \square A$

These results show that the system $\mathbf{N} \mathbf{J}_{\mathbf{I S} 4}$ is equivalent to the axiomatization $\mathbf{A}_{\mathbf{I S} 4}$, so it is reasonable to choose $\mathbf{N J}_{\mathbf{I S} 4}$ as a foundation on which to develop proof search strategies for IS4.

While Girard, Lafont, and Taylor suggest that we should think of natural deductions as the "true 'proof' objects" [10], natural deduction systems have traditionally not seen much use as formalisms for proof search, mainly as a result of their lack of syntax-directedness. The first step towards being able to perform proof search over natural deductions is to restrict the proof space to those natural deductions that are in normal form. We formalize this restriction in the next section.

\section{Normalized Natural Deduction}

$\mathbf{N J}_{\text {IS4 }}$ is a possible starting point for proof search strategies, but a better idea is to restrict the proof space described by $\mathbf{N} \mathbf{J}_{\mathbf{I S} \mathbf{4}}$ in such a way that only natural deductions in normal form can be constructed. This is achieved by annotating judgements with their intended direction of reasoning:

$$
\begin{array}{ll}
\Delta ; \Gamma \vdash A \uparrow & A \text { has a normal proof under hypotheses } \Delta \text { and } \Gamma, \\
\Delta ; \Gamma \vdash A \downarrow & A \text { can be extracted from hypotheses in } \Delta \text { and } \Gamma \text { using } \\
& \text { only elimination rules. }
\end{array}
$$

The resulting system, which we will call $\mathbf{N} \mathbf{J}_{\mathbf{I S 4}}^{\mathbf{N}}$, is shown in Fig. 3. As in $\mathbf{N J}_{\mathbf{I S 4} \text {, }}$, the contexts of this system are formally ordered lists, but $\mathbf{N} \mathbf{J}_{\mathbf{I S} 4}^{\mathbf{N}}$ also has the structural properties of weakening, contraction, and exchange for both contexts. Again, we will generally think of contexts in $\mathbf{N J}_{\mathbf{I S} 4}^{\mathbf{N}}$ as multisets. 


$$
\begin{aligned}
& \overline{\Delta ; \Gamma_{1}, A, \Gamma_{2} \vdash A \downarrow} \operatorname{hyp}_{1} \frac{}{\Delta_{1}, A, \Delta_{2} ; \Gamma \vdash A \downarrow} \operatorname{hyp}_{2} \frac{\Delta ; \Gamma \vdash \perp \downarrow}{\Delta ; \Gamma \vdash A \uparrow} \perp \mathrm{E} \\
& \frac{\Delta ; \Gamma, A_{1} \vdash A_{2} \uparrow}{\Delta ; \Gamma \vdash A_{1} \supset A_{2} \uparrow} \supset \mathrm{I} \quad \frac{\Delta ; \Gamma \vdash A_{1} \supset A_{2} \downarrow \quad \Delta ; \Gamma \vdash A_{1} \uparrow}{\Delta ; \Gamma \vdash A_{2} \downarrow} \supset \mathrm{E} \\
& \frac{\Delta ; \Gamma \vdash A_{1} \uparrow \quad \Delta ; \Gamma \vdash A_{2} \uparrow}{\Delta ; \Gamma \vdash A_{1} \wedge A_{2} \uparrow} \wedge \mathrm{I} \frac{\Delta ; \Gamma \vdash A_{1} \wedge A_{2} \downarrow}{\Delta ; \Gamma \vdash A_{j} \downarrow} \wedge \mathrm{E}_{j} \\
& \frac{\Delta ; \Gamma \vdash A_{j} \uparrow}{\Delta ; \Gamma \vdash A_{1} \vee A_{2} \uparrow} \vee \mathrm{l}_{j} \frac{\Delta ; \Gamma \vdash A_{1} \vee A_{2} \downarrow \quad \Delta ; \Gamma, A_{1} \vdash C \uparrow \quad \Delta ; \Gamma, A_{2} \vdash C \uparrow}{\Delta ; \Gamma \vdash C \uparrow} \vee \mathrm{E} \\
& \frac{\Delta ; \cdot \vdash A \uparrow}{\Delta ; \Gamma \vdash \square A \uparrow} \square \quad \text { । } \frac{\Delta ; \Gamma \vdash \square A \downarrow \quad \Delta, A ; \Gamma \vdash C \uparrow}{\Delta ; \Gamma \vdash C \uparrow} \square \mathrm{E} \\
& \frac{\Delta ; \Gamma \vdash A \uparrow}{\Delta ; \Gamma \vdash \diamond A \uparrow} \diamond \mathrm{I} \quad \frac{\Delta ; \Gamma \vdash \diamond A \downarrow \quad \Delta ; A \vdash \diamond C \uparrow}{\Delta ; \Gamma \vdash \diamond C \uparrow} \diamond \mathrm{E} \\
& \frac{\Delta ; \Gamma \vdash A \downarrow}{\Delta ; \Gamma \vdash A \uparrow} \uparrow \downarrow \quad(A \text { is atomic }) \\
& j \in\{1,2\}
\end{aligned}
$$

Fig. 3. $\mathrm{NJ}_{\mathrm{IS} 4}^{\mathrm{N}}$

\section{Lemma 12 (Weakening for $\mathrm{NJ}_{\mathrm{IS} 4}^{\mathrm{N}}$ ).}
1. If $\Delta ; \Gamma_{1}, \stackrel{\mathcal{D}}{\Gamma_{2}} \vdash C \uparrow$, then $\Delta ; \Gamma_{1}, A, \Gamma_{2} \vdash C \uparrow$.
2. If $\Delta ; \Gamma_{1}, \stackrel{\mathcal{E}}{\Gamma_{2}} \vdash C \downarrow$, then $\Delta ; \Gamma_{1}, A, \Gamma_{2} \vdash C \downarrow$.

Proof. By simultaneous induction on the structure of the given derivation.

\section{Lemma 13 (Modal weakening for $\mathrm{NJ}_{\mathrm{IS} 4}^{\mathrm{N}}$ ).}

$\mathcal{D}$
1. If $\Delta_{1}, \Delta_{2} ; \Gamma \vdash C \uparrow$, then $\Delta_{1}, A, \Delta_{2} ; \Gamma \vdash C \uparrow$.
2. If $\Delta_{1}, \Delta_{2} ; \Gamma \vdash C \downarrow$, then $\Delta_{1}, A, \Delta_{2} ; \Gamma \vdash C \downarrow$.

Proof. By simultaneous induction on the structure of the given derivation.

The substitution properties of $\mathbf{N J}_{\mathbf{I S} 4}^{\mathbf{N}}$ are similar to those of $\mathbf{N J}_{\mathbf{I S} 4}$, but take the direction of reasoning into account.

\section{Lemma 14 (Substitution property for $\mathrm{NJ}_{\mathrm{IS} 4}^{\mathrm{N}}$ ).}

$\mathcal{D}$
1. If $\Delta ; \Gamma_{1}, A, \Gamma_{2} \vdash C \uparrow$ and $\Delta ; \Gamma_{1}, \Gamma_{2} \vdash A \downarrow$, then $\Delta ; \Gamma_{1}, \Gamma_{2} \vdash C \uparrow$.
2. If $\Delta ; \Gamma_{1}, A, \Gamma_{2} \vdash C \downarrow$ and $\Delta ; \Gamma_{1}, \Gamma_{2} \vdash A \downarrow$, then $\Delta ; \Gamma_{1}, \Gamma_{2} \vdash C \downarrow$. 
Proof. By simultaneous induction on the structure of the first given derivation, using Lemmas 12 and 13 where needed.

\section{Lemma 15 (Modal substitution property for $\mathrm{NJ}_{\mathrm{IS} 4}^{\mathrm{N}}$ ).}

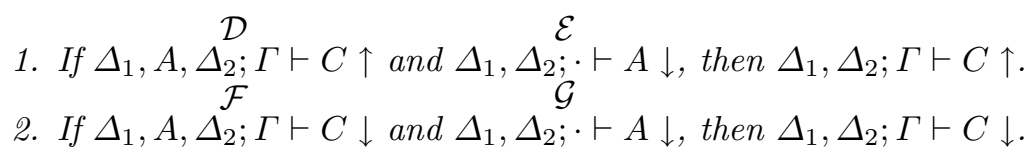

Proof. By simultaneous induction on the structure of the first given derivation, using Lemmas 12 and 13 where needed.

Note that while $\mathbf{N} \mathbf{J}_{\mathbf{I S} 4}^{\mathbf{N}}$ requires the principal formula of the coercion rule $\uparrow \downarrow$ to be atomic, the following result shows that a corresponding unconstrained rule is admissible.

\section{Lemma 16 (Admissibility of nonatomic coercion for $\mathrm{NJ}_{\mathrm{IS} 4}^{\mathrm{N}}$ ).}

If $\Delta ; \Gamma \vdash A \downarrow$, then $\Delta ; \Gamma \vdash A \uparrow$.

Proof. By induction on the size of $A$. We show the base cases and the cases involving modalities.

Case: $A$ is atomic.

(1) $\Delta ; \Gamma \vdash A \uparrow$

Case: $A=\perp$.

(1) $\Delta ; \Gamma \vdash \perp \uparrow$

$\perp \mathrm{E}(\mathcal{D})$

Case: $A=\square A_{1}$.

(1) $\Delta, A_{1} ; \cdot \vdash A_{1} \downarrow$

(2) $\Delta, A_{1} ; \cdot \vdash A_{1} \uparrow$

(3) $\Delta, A_{1} ; \Gamma \vdash \square A_{1} \uparrow$

(4) $\Delta ; \Gamma \vdash \square A_{1} \uparrow$

$\square \mathrm{E}(\mathcal{D}, 3)$

Case: $A=\diamond A_{1}$.

(1) $\Delta ; A_{1} \vdash A_{1} \downarrow$

(2) $\Delta ; A_{1} \vdash A_{1} \uparrow$

(3) $\Delta ; A_{1} \vdash \diamond A_{1} \uparrow$

(4) $\Delta ; \Gamma \vdash \diamond A_{1} \uparrow$

Before continuing, it is important to establish that $\mathbf{N J}_{\mathbf{I S} \mathbf{4}}^{\mathbf{N}}$ is equivalent, in terms of provability, to $\mathbf{N J}_{\mathbf{I S} 4}$. The soundness of $\mathbf{N J}_{\mathbf{I S} 4}^{\mathbf{N}}$ with respect to $\mathbf{N J}_{\mathbf{I S} 4}$ is easy to show.

\section{Theorem 5 (Soundness of $\mathrm{NJ}_{\mathrm{IS} 4}^{\mathrm{N}}$ with respect to $\mathrm{NJ}_{\mathrm{IS} 4}$ ).}
1. If $\Delta ; \Gamma \vdash A \uparrow$, then $\Delta ; \Gamma \vdash A$.
2. If $\Delta ; \Gamma \vdash A \downarrow$, then $\Delta ; \Gamma \vdash A$. 


$$
\begin{gathered}
\frac{\Delta ; \Gamma_{1}, A, \Gamma_{2} \Rightarrow A}{\text { init }} \quad(A \text { is atomic }) \quad \overline{\Delta ; \Gamma_{1}, \perp, \Gamma_{2} \Rightarrow A} \perp \mathrm{L} \\
\frac{\Delta_{1}, A, \Delta_{2} ; \Gamma, A \Rightarrow C}{\Delta_{1}, A, \Delta_{2} ; \Gamma \Rightarrow C} \text { refl } \\
\frac{\Delta ; \Gamma, A_{1} \Rightarrow A_{2}}{\Delta ; \Gamma \Rightarrow A_{1} \supset A_{2}} \supset \mathrm{R} \frac{\Delta ; \Gamma_{1}, A_{1} \supset A_{2}, \Gamma_{2} \Rightarrow A_{1} \quad \Delta ; \Gamma_{1}, A_{1} \supset A_{2}, \Gamma_{2}, A_{2} \Rightarrow C}{\Delta ; \Gamma_{1}, A_{1} \supset A_{2}, \Gamma_{2} \Rightarrow C} \supset \mathrm{L} \\
\frac{\Delta ; \Gamma \Rightarrow A_{1} \quad \Delta ; \Gamma \Rightarrow A_{2}}{\Delta ; \Gamma \Rightarrow A_{1} \wedge A_{2}} \wedge \mathrm{R} \frac{\Delta ; \Gamma_{1}, A_{1} \wedge A_{2}, \Gamma_{2}, A_{j} \Rightarrow C}{\Delta ; \Gamma_{1}, A_{1} \wedge A_{2}, \Gamma_{2} \Rightarrow C} \wedge \mathrm{L}_{j} \\
\frac{\Delta ; \Gamma \Rightarrow A_{j}}{\Delta ; \Gamma \Rightarrow A_{1} \vee A_{2}} \vee \mathrm{R}_{j} \frac{\Delta ; \Gamma_{1}, A_{1} \vee A_{2}, \Gamma_{2}, A_{1} \Rightarrow C \quad \Delta ; \Gamma_{1}, A_{1} \vee A_{2}, \Gamma_{2}, A_{2} \Rightarrow C}{\Delta ; \Gamma_{1}, A_{1} \vee A_{2}, \Gamma_{2} \Rightarrow C} \\
\frac{\Delta ; ; \Rightarrow A}{\Delta ; \Gamma \Rightarrow \square A} \square \mathrm{R} \quad \frac{\Delta, A ; \Gamma_{1}, \square A, \Gamma_{2} \Rightarrow C}{\Delta ; \Gamma_{1}, \square A, \Gamma_{2} \Rightarrow C} \square \mathrm{L} \\
\frac{\Delta ; \Gamma \Rightarrow A}{\Delta ; \Gamma \Rightarrow \diamond A} \diamond \mathrm{R} \frac{\Delta ; A \Rightarrow \diamond C}{\Delta ; \Gamma_{1}, \diamond A, \Gamma_{2} \Rightarrow \diamond C} \diamond \mathrm{L} \\
j \in\{1,2\}
\end{gathered}
$$

Fig. 4. $\mathrm{LJ}_{\mathrm{IS} 4}$

Proof. By simultaneous induction on the structure of the first given derivation.

The proof of the completeness of $\mathbf{N J}_{\mathbf{I S} 4}^{\mathbf{N}}$ with respect to $\mathbf{N J}_{\mathbf{I S} 4}$ is not as immediate, but still routine. We will develop a number of intermediate results, giving the final completeness result as Theorem 10. Our proof is by cut-elimination in an unfocused sequent calculus system designed to be equivalent to $\mathbf{N J}_{\mathbf{I S} 4}$. This system, which we call $\mathbf{L} \mathbf{J}_{\mathbf{I S} \mathbf{4}}$, is shown in Fig. 4 and features sequents of the the form $\Delta ; \Gamma \Rightarrow A$. Note that $\mathbf{L} \mathbf{J}_{\mathbf{I S} 4}$ is very similar to a sequent calculus presented by Nanevski, Pfenning, and Pientka for contextual modal logic [15], but is not the sequent calculus system that we will use for proof search. It is purely a technical device that we introduce for the purpose of the proof of completeness of $\mathbf{N J}_{\mathbf{I S} 4}^{\mathbf{N}}$ with respect to $\mathbf{N J}_{\mathbf{I S} 4}$. The cut rule

$$
\frac{\Delta ; \Gamma_{1}, A, \Gamma_{2} \Rightarrow C \quad \Delta ; \Gamma_{1}, \Gamma_{2} \Rightarrow A}{\Delta ; \Gamma_{1}, \Gamma_{2} \Rightarrow C} \text { cut }
$$

is not part of $\mathbf{L} \mathbf{J}_{\mathbf{I S} \mathbf{4}}$, but we will refer to the system obtained by augmenting $\mathbf{L J}_{\mathbf{I S} \mathbf{4}}$ by the rule cut as $\mathbf{L} \mathbf{J}_{\mathbf{I S} \mathbf{4}}^{+}$. To distinguish between sequents of $\mathbf{L} \mathbf{J}_{\mathbf{I S} \mathbf{4}}$ and $\mathbf{L} \mathbf{J}_{\mathbf{I S} 4}^{+}$, we will decorate the arrows of $\mathbf{L} \mathbf{J}_{\mathbf{I S} \mathbf{4}}^{+}$sequents with a + sign, viz. $\Delta ; \Gamma \stackrel{+}{\Rightarrow}$ A.

Note that while the init rules of $\mathbf{L} \mathbf{J}_{\mathbf{I S} 4}$ and $\mathbf{L} \mathbf{J}_{\mathbf{I S} 4}^{+}$are required to have atomic principal formulas, unconstrained variants are admissible, as shown by the following result. 
Lemma 17 (Admissibility of nonatomic init for $\mathbf{L J}_{\mathbf{I S} 4}$ ).

$\Delta ; \Gamma_{1}, A, \Gamma_{2} \Rightarrow A$.

Proof. By induction on the size of $A$. We show the base cases and the cases involving modalities.

Case: $A$ is atomic.

(1) $\Delta ; \Gamma_{1}, A, \Gamma_{2} \Rightarrow A \quad$ init

Case: $A=\perp$.

(1) $\Delta ; \Gamma_{1}, \perp, \Gamma_{2} \Rightarrow \perp \quad \perp \mathrm{L}$

Case: $A=\square A_{1}$.

(1) $\Delta, A_{1} ; A_{1} \Rightarrow A_{1}$ i.h.

(2) $\Delta, A_{1} ; \cdot \Rightarrow A_{1} \quad$ refl (1)

(3) $\Delta, A_{1} ; \Gamma_{1}, \square A_{1}, \Gamma_{2} \Rightarrow \square A_{1} \quad \square \mathrm{R}(2)$

(4) $\Delta ; \Gamma_{1}, \square A_{1}, \Gamma_{2} \Rightarrow \square A_{1} \quad \square \mathrm{L}(3)$

Case: $A=\diamond A_{1}$.

(1) $\Delta ; A_{1} \Rightarrow A_{1}$ i.h.

(2) $\Delta ; A_{1} \Rightarrow \diamond A_{1} \quad \diamond \mathrm{R}(1)$

(3) $\Delta ; \Gamma_{1}, \diamond A_{1}, \Gamma_{2} \Rightarrow \diamond A_{1} \quad \diamond \mathrm{L}(2)$

Lemma 18 (Admissibility of nonatomic init for $\mathbf{L J}_{\mathrm{IS} 4}^{+}$).

$\Delta ; \Gamma_{1}, A, \Gamma_{2} \stackrel{+}{\Rightarrow} A$.

Proof. By induction on the size of $A$. The cases are analogous to those in the proof of Lemma 17.

Another system we will introduce for this completeness proof is $\mathbf{N J}_{\mathbf{I S} \mathbf{4}}^{\mathbf{N}}$, which is obtained from $\mathbf{N J}_{\mathbf{I S} \mathbf{4}}^{\mathbf{N}}$ by augmenting it by the rule

$$
\frac{\Delta ; \Gamma \vdash A \uparrow}{\Delta ; \Gamma \vdash A \downarrow} \downarrow \uparrow
$$

Unlike the regular coercion rule $\uparrow \downarrow$, the principal formula of this rule need not be atomic. To distinguish between the judgements of $\mathbf{N J}_{\mathbf{I S} 4}^{\mathbf{N}+}$ and $\mathbf{N} \mathbf{J}_{\mathbf{I S} 4}^{\mathbf{N}}$, we will decorate the turnstiles of $\mathbf{N J}_{\text {IS4 }}^{\mathbf{N}+}$ judgements with a + sign, viz. $\Delta ; \Gamma \vdash_{+} A$. As with $\mathbf{N J}_{\mathbf{I S} 4}^{\mathbf{N}}$, an unconstrained regular coercion rule is admissible for $\mathbf{N} \mathbf{J}_{\mathbf{I S} 4}^{\mathbf{N}+}$.

Lemma 19 (Admissibility of nonatomic coercion for $\mathrm{NJ}_{\mathrm{IS} 4}^{\mathrm{N}+}$ ).

If $\Delta ; \Gamma \stackrel{\mathcal{D}}{\vdash_{+}} A \downarrow$, then $\Delta ; \Gamma \vdash_{+} A \uparrow$.

Proof. By induction on the size of $A$. The cases are analogous to those in the proof of Lemma 16.

We can now prove the first intermediate result needed for the proof of completeness of $\mathbf{N J}_{\mathbf{I S} 4}^{\mathbf{N}}$ with respect to $\mathbf{N J}_{\mathbf{I S} 4}$.

Theorem 6 (Completeness of $\mathrm{NJ}_{\mathrm{IS} 4}^{\mathrm{N}+}$ with respect to $\mathrm{NJ}_{\mathrm{IS} 4}^{\mathrm{N}}$ ). 


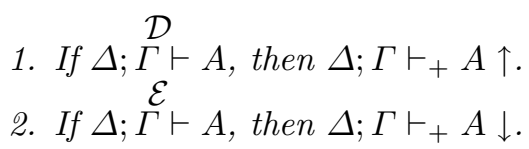

Proof. By simultaneous induction on the structure of the given derivation, using Lemma 19 and the rule $\downarrow \uparrow$ where needed.

For the next few results, we require the usual structural properties of both $\mathbf{L J}_{\mathbf{I S} 4}$ and $\mathbf{L} \mathbf{J}_{\mathbf{I S} \mathbf{4}}^{+}$.

Lemma 20 (Weakening for $\mathbf{L J}_{\mathrm{IS} 4}$ ).

If $\Delta ; \Gamma_{1}, \Gamma_{2} \Rightarrow C$, then $\Delta ; \Gamma_{1}, A, \Gamma_{2} \Rightarrow C$.

Proof. By induction on the structure of $\mathcal{D}$.

Lemma 21 (Modal weakening for $\mathrm{LJ}_{\mathrm{IS} 4}$ ).

If $\Delta_{1}, \stackrel{\mathcal{D}}{\mathcal{D}}, \Gamma \Rightarrow C$, then $\Delta_{1}, A, \Delta_{2} ; \Gamma \Rightarrow C$.

Proof. By induction on the structure of $\mathcal{D}$.

Lemma 22 (Exchange for $\mathrm{LJ}_{\mathrm{IS} 4}$ ).

If $\Delta ; \Gamma_{1}, A_{1}, \Gamma_{2}, A_{2}, \Gamma_{3} \Rightarrow C$, then $\Delta ; \Gamma_{1}, A_{2}, \Gamma_{2}, A_{1}, \Gamma_{3} \Rightarrow C$.

Proof. By induction on the structure of $\mathcal{D}$.

Lemma 23 (Modal exchange for $\mathrm{LJ}_{\mathrm{IS} 4}$ ).

If $\Delta_{1}, A_{1}, \Delta_{2}, \stackrel{\mathcal{D}}{A_{2}}, \Delta_{3} ; \Gamma \Rightarrow C$, then $\Delta_{1}, A_{2}, \Delta_{2}, A_{1}, \Delta_{3} ; \Gamma \Rightarrow C$.

Proof. By induction on the structure of $\mathcal{D}$.

Lemma 24 (Contraction for $\mathbf{L J}_{\mathrm{IS} 4}$ ).

If $\Delta ; \Gamma_{1}, A, \Gamma_{2}^{\mathcal{D}}, A, \Gamma_{3} \Rightarrow C$, then $\Delta ; \Gamma_{1}, A, \Gamma_{2}, \Gamma_{3} \Rightarrow C$.

Proof. By induction on the structure of $\mathcal{D}$.

Lemma 25 (Modal contraction for $\mathrm{LJ}_{\mathrm{IS} 4}$ ).

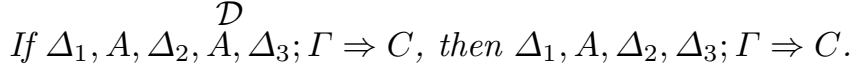

Proof. By induction on the structure of $\mathcal{D}$.

All of the structural properties also hold for $\mathbf{L} \mathbf{J}_{\mathbf{I S} 4}^{+}$.

Lemma 26 (Weakening for $\mathrm{LJ}_{\mathrm{IS} 4}^{+}$).

$\mathcal{D}$

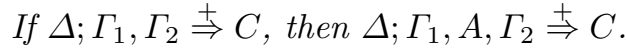

Proof. By induction on the structure of $\mathcal{D}$. 
Lemma 27 (Modal weakening for $\mathrm{LJ}_{\mathrm{IS} 4}^{+}$).

$\mathcal{D}$

If $\Delta_{1}, \Delta_{2} ; \Gamma \stackrel{+}{\Rightarrow} C$, then $\Delta_{1}, A, \Delta_{2} ; \Gamma \stackrel{+}{\Rightarrow} C$.

Proof. By induction on the structure of $\mathcal{D}$.

Lemma 28 (Exchange for $\mathrm{LJ}_{\mathrm{IS} 4}^{+}$).

$$
\mathcal{D}
$$

If $\Delta ; \Gamma_{1}, A_{1}, \Gamma_{2}, A_{2}, \Gamma_{3} \stackrel{+}{\Rightarrow} C$, then $\Delta ; \Gamma_{1}, A_{2}, \Gamma_{2}, A_{1}, \Gamma_{3} \stackrel{+}{\Rightarrow} C$.

Proof. By induction on the structure of $\mathcal{D}$.

Lemma 29 (Modal exchange for $\mathrm{LJ}_{\mathrm{IS} 4}^{+}$).

$\mathcal{D}$

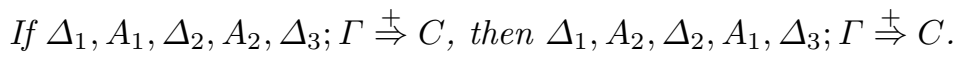

Proof. By induction on the structure of $\mathcal{D}$.

Lemma 30 (Contraction for $\mathrm{LJ}_{\mathrm{IS} 4}^{+}$).

$\mathcal{D}$

If $\Delta ; \Gamma_{1}, A, \Gamma_{2}, A, \Gamma_{3} \stackrel{+}{\Rightarrow} C$, then $\Delta ; \Gamma_{1}, A, \Gamma_{2}, \Gamma_{3} \stackrel{+}{\Rightarrow} C$.

Proof. By induction on the structure of $\mathcal{D}$.

Lemma 31 (Modal contraction for $\mathrm{LJ}_{\mathrm{IS} 4}^{+}$).

$\mathcal{D}$

If $\Delta_{1}, A, \Delta_{2}, A, \Delta_{3} ; \Gamma \stackrel{+}{\Rightarrow} C$, then $\Delta_{1}, A, \Delta_{2}, \Delta_{3} ; \Gamma \stackrel{+}{\Rightarrow} C$.

Proof. By induction on the structure of $\mathcal{D}$.

Moreover, the proofs of these structural properties for both $\mathbf{L} \mathbf{J}_{\mathbf{I S} 4}$ and $\mathbf{L} \mathbf{J}_{\text {IS4 }}^{+}$ show that applying any form of weakening, exchange, or contraction to a derivation does not affect its structure. This is an important observation that will be used tacitly in the proof of the admissibility of the cut rule for $\mathbf{L} \mathbf{J}_{\mathbf{I S} \mathbf{4}}$, Lemma 33.

First, however, we can prove the next key result on the way to Theorem 10.

Theorem 7 (Completeness of $\mathrm{LJ}_{\text {IS4 }}^{+}$with respect to $\mathrm{NJ}_{\mathrm{IS} 4}^{\mathrm{N}+}$ ).

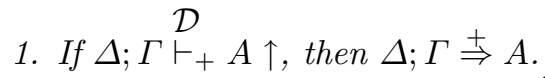

2. If $\Delta ; \Gamma_{1}, \stackrel{\mathcal{E}}{\Gamma_{2}} \vdash_{+} A \downarrow$ and $\Delta ; \Gamma_{1}, A, \Gamma_{2} \stackrel{\mathcal{F}}{\Rightarrow} C$, then $\Delta ; \Gamma_{1}, \Gamma_{2} \stackrel{+}{\Rightarrow} C$.

Proof. By simultaneous induction on the structure of the first given derivation. We show the base cases and the cases involving modalities.

Case:

$$
\mathcal{E}=\overline{\Delta ; \Gamma_{1}, \Gamma_{2} \vdash_{+} A \downarrow} \operatorname{hyp}_{1} \quad\left(A \in \Gamma_{1}, \Gamma_{2}\right)
$$

(1) $\Delta ; \Gamma_{1}, \Gamma_{2} \stackrel{+}{\Rightarrow} C$

Lemma $30(\mathcal{F})$ 


\section{Case:}

$$
\begin{array}{lr}
\mathcal{E}=\overline{\Delta_{1}, A, \Delta_{2} ; \Gamma_{1}, \Gamma_{2} \vdash_{+} A \downarrow} \text { hyp }_{2} & \\
\text { (1) } \Delta_{1}, A, \Delta_{2} ; \Gamma_{1}, \Gamma_{2}, A \stackrel{+}{\Rightarrow} C & \text { Lemma } 28(\mathcal{F}) \\
\text { (2) } \Delta_{1}, A, \Delta_{2} ; \Gamma_{1}, \Gamma_{2} \stackrel{\Rightarrow}{\Rightarrow} C & \text { refl }(1)
\end{array}
$$

$$
\mathcal{D}=\frac{\mathcal{D}_{1}}{\Delta ; \vdash_{+} A \uparrow}
$$

(1) $\Delta ; \stackrel{+}{\Rightarrow} A$

i.h. $\left(\mathcal{D}_{1}\right)$

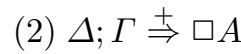

Case:

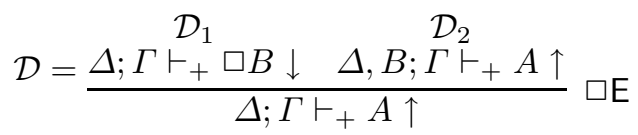
(1) $\Delta, B ; \Gamma \stackrel{+}{\Rightarrow} A$
(2) $\Delta, B ; \Gamma, \square B \stackrel{+}{\Rightarrow} A$
(3) $\Delta ; \Gamma, \square B \stackrel{+}{\Rightarrow} A$
(4) $\Delta ; \Gamma \stackrel{+}{\Rightarrow} A$

i.h. $\left(\mathcal{D}_{2}\right)$

Lemma 26 (1)

$\square \mathrm{L}(2)$

i.h. $\left(\mathcal{D}_{1}, 3\right)$

Case:

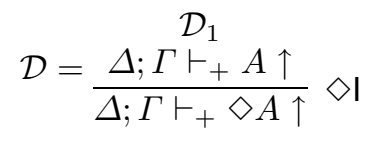

(1) $\Delta ; \Gamma \stackrel{+}{\Rightarrow} A$

(2) $\Delta ; \Gamma \stackrel{े}{\Rightarrow} \diamond A$

i.h. $\left(\mathcal{D}_{1}\right)$ $\diamond \mathrm{R}(1)$

\section{Case:}

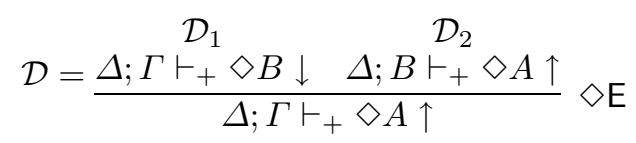

(1) $\Delta ; B \stackrel{+}{\Rightarrow} \diamond A$

i.h. $\left(\mathcal{D}_{2}\right)$

(2) $\Delta ; \Gamma, \diamond B \stackrel{+}{\Rightarrow} \diamond A$

(3) $\Delta ; \Gamma \stackrel{+}{\Rightarrow} \diamond A$

i.h. $\left(\mathcal{D}_{1}, 2\right)$

Case:

$$
\mathcal{D}=\frac{\mathcal{D}_{1}}{\Delta ; \Gamma \vdash_{+} A \downarrow} \uparrow \uparrow
$$

(1) $\Delta ; \Gamma, A \stackrel{+}{\Rightarrow} A$

(2) $\Delta ; \Gamma \stackrel{+}{\Rightarrow} A$

i.h. $\left(\mathcal{D}_{1}, 1\right)$

\section{Case:}

$$
\mathcal{E}=\frac{\Delta ; \Gamma \vdash_{+} A \uparrow}{\Delta ; \Gamma \vdash_{+} A \downarrow} \downarrow \uparrow
$$


(1) $\Delta ; \Gamma \stackrel{+}{\Rightarrow} A$

i.h. $\left(\mathcal{E}_{1}\right)$

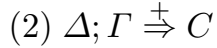

cut $(\mathcal{F}, 1)$

The following intermediate result will be used in the proof of the admissibility of the cut rule for $\mathbf{L} \mathbf{J}_{\mathbf{I S} 4}$, Lemma 33. It effectively establishes the admissibility of cut when used in a very particular way.

Lemma 32 ( $\perp$-cutting for $\left.\mathbf{L J}_{\mathrm{IS} 4}\right)$.

If $\Delta ; \stackrel{\mathcal{D}}{\Gamma} \perp$, then $\Delta ; \Gamma \Rightarrow A$.

Proof. By induction on the structure of $\mathcal{D}$.

We are now able to prove the fundamental cut-elimination result that we need.

\section{Lemma 33 (Admissibility of cut for $\mathrm{LJ}_{\mathrm{IS} 4}$ ).}

$$
\begin{aligned}
& \text { D } \\
& \text { 1. (Cut) If } \Delta ; \Gamma_{1}, A, \Gamma_{2} \Rightarrow C \text { and } \Delta ; \Gamma_{1}, \Gamma_{2}^{\mathcal{E}} \Rightarrow \underset{\mathcal{F}}{A \text {, then }} \Delta ; \Gamma_{1}, \Gamma_{2} \Rightarrow C \text {. } \\
& \text { 2. (Modal cut) If } \Delta_{1}, A, \Delta_{2} ; \Gamma \Rightarrow C \text { and } \Delta_{1}, \Delta_{1} ; \cdot \Rightarrow A \text {, then } \Delta_{1}, \Delta_{2} ; \Gamma \Rightarrow C \text {. }
\end{aligned}
$$

Proof. By simultaneous nested induction. Our induction variables are triples of the form $\left\langle\right.$ size, case, height $t_{1}+$ height $\left._{2}\right\rangle$, where size is the size of the cut formula $A$, computed inductively in the usual way, case is 1 or 2 , depending on which induction case (cut or modal cut) we are considering, and height $t_{1}$ and height $_{2}$ are the heights of the two given derivations. We let $\left\langle v_{1}, \ldots, v_{n}\right\rangle\left\langle\left\langle w_{1}, \ldots, w_{n}\right\rangle\right.$ if there is an $i \in\{1, \ldots, n\}$ such that $v_{j}=w_{j}$ for $j<i$ and $v_{i}<w_{i}$. We show the base cases and the cases involving modalities.

At the top level, the cases of the proof are in two categories, those for cut and those for modal cut. We first give the cases for cut, where we can only appeal to the induction hypothesis for modal cut if the cut formula decreases in size.

Case: Either $\mathcal{D}$ or $\mathcal{E}$ ends with an application of init, $\perp \mathrm{L}$, or refl.

\section{Subcase:}

$$
\mathcal{D}=\overline{\Delta ; \Gamma_{1}, A, \Gamma_{2} \Rightarrow C} \text { init } \quad(A=C)
$$

(1) $\Delta ; \Gamma_{1}, \Gamma_{2} \Rightarrow C$

Equality $(\mathcal{E})$

\section{Subcase:}

$$
\mathcal{D}=\overline{\Delta ; \Gamma_{1}, A, \Gamma_{2} \Rightarrow C} \text { init } \quad\left(A \neq C, C \in \Gamma_{1}, \Gamma_{2}\right)
$$

(1) $\Delta ; \Gamma_{1}, \Gamma_{2} \Rightarrow C$

Lemma 17

\section{Subcase:}

$$
\mathcal{E}=\overline{\Delta ; \Gamma_{1}, \Gamma_{2} \Rightarrow A} \text { init } \quad\left(A \in \Gamma_{1}, \Gamma_{2}\right)
$$

(1) $\Delta ; \Gamma_{1}, \Gamma_{2} \Rightarrow C$

Lemma $24(\mathcal{D})$ 


\section{Subcase:}

$$
\mathcal{D}={\overline{\Delta ; \Gamma_{1}, A, \Gamma_{2} \Rightarrow C}}^{\perp \mathrm{L}} \quad(A=\perp)
$$

(1) $\Delta ; \Gamma_{1}, \Gamma_{2} \Rightarrow \perp$

Equality $(\mathcal{E})$

(2) $\Delta ; \Gamma_{1}, \Gamma_{2} \Rightarrow C$

Subcase:

$$
\mathcal{D}={\overline{\Delta ; \Gamma_{1}, A, \Gamma_{2} \Rightarrow C}}^{\perp \mathrm{L}} \quad\left(A \neq \perp, \perp \in \Gamma_{1}, \Gamma_{2}\right)
$$

(1) $\Delta ; \Gamma_{1}, \Gamma_{2} \Rightarrow C$

Subcase:

$$
\mathcal{E}=\overline{\Delta ; \Gamma_{1}, \Gamma_{2} \Rightarrow A} \perp \mathrm{L} \quad\left(\perp \in \Gamma_{1}, \Gamma_{2}\right)
$$

(1) $\Delta ; \Gamma_{1}, \Gamma_{2} \Rightarrow C$

Subcase:

$\mathcal{D}_{1}$

$$
\mathcal{D}=\frac{\Delta_{1}, B, \Delta_{2} ; \Gamma_{1}, A, \Gamma_{2}, B \Rightarrow C}{\Delta_{1}, B, \Delta_{2} ; \Gamma_{1}, A, \Gamma_{2} \Rightarrow C} \text { refl }
$$

(1) $\Delta_{1}, B, \Delta_{2} ; \Gamma_{1}, \Gamma_{2}, B \Rightarrow A$

(2) $\Delta_{1}, B, \Delta_{2} ; \Gamma_{1}, \Gamma_{2}, B \Rightarrow C$

(3) $\Delta_{1}, B, \Delta_{2} ; \Gamma_{1}, \Gamma_{2} \Rightarrow C$

Subcase:

$$
\mathcal{E}=\frac{\Delta_{1}, B, \Delta_{2} ; \Gamma_{1}, \Gamma_{2}, B \Rightarrow A}{\Delta_{1}, B, \Delta_{2} ; \Gamma_{1}, \Gamma_{2} \Rightarrow A} \text { refl }
$$

(1) $\Delta_{1}, B, \Delta_{2} ; \Gamma_{1}, A, \Gamma_{2}, B \Rightarrow C$

(2) $\Delta_{1}, B, \Delta_{2} ; \Gamma_{1}, \Gamma_{2}, B \Rightarrow C$

(3) $\Delta_{1}, B, \Delta_{2} ; \Gamma_{1}, \Gamma_{2} \Rightarrow C$

Lemma $20(\mathcal{D})$

i.h. $\left(1, \mathcal{E}_{1}\right)$ refl $(2)$

Case: The cut formula is the principal formula of the last inference of both $\mathcal{D}$ and $\mathcal{E}$.

Subcase:

$$
\mathcal{D}=\frac{\mathcal{D}_{1}, A ; \Gamma_{1}, \square A, \Gamma_{2} \Rightarrow C}{\Delta ; \Gamma_{1}, \square A, \Gamma_{2} \Rightarrow C} \square \mathrm{L} \quad \text { and } \quad \mathcal{E}=\frac{\mathcal{E}_{1} ; \cdot \Rightarrow A}{\Delta ; \Gamma_{1}, \Gamma_{2} \Rightarrow \square A} \square \mathrm{R}
$$

(1) $\Delta, A ; \Gamma_{1}, \Gamma_{2} \Rightarrow \square A$

Lemma $21(\mathcal{E})$

(2) $\Delta, A ; \Gamma_{1}, \Gamma_{2} \Rightarrow C$

(3) $\Delta ; \Gamma_{1}, \Gamma_{2} \Rightarrow C$

i.h. $\left(\mathcal{D}_{1}, 1\right)$

i.h. $\left(2, \mathcal{E}_{1}\right)$

Subcase:

$$
\mathcal{D}=\frac{\Delta ; A \Rightarrow \diamond C}{\Delta ; \Gamma_{1}, \diamond A, \Gamma_{2} \Rightarrow \diamond C} \diamond \mathrm{L} \quad \text { and } \quad \mathcal{E}=\frac{\Delta ; \Gamma_{1}, \Gamma_{2} \Rightarrow A}{\Delta ; \Gamma_{1}, \Gamma_{2} \Rightarrow \diamond A} \diamond \mathrm{R}
$$

(1) $\Delta ; \Gamma_{1}, \Gamma_{2}, A \Rightarrow \diamond C$

Lemma $20\left(\mathcal{D}_{1}\right)$

(2) $\Delta ; \Gamma_{1}, \Gamma_{2} \Rightarrow \diamond C$

i.h. $\left(1, \mathcal{E}_{1}\right)$

Case: The cut formula is not the principal formula of the last inference of $\mathcal{D}$. 


\section{Subcase:}

$$
\mathcal{D}=\frac{\mathcal{D}_{1}}{\Delta ; \cdot \stackrel{\Rightarrow}{\Rightarrow} C} \frac{\mathrm{R}}{\Gamma_{1}, A, \Gamma_{2} \Rightarrow \square C} \square
$$

(1) $\Delta ; \Gamma_{1}, \Gamma_{2} \Rightarrow \square C$

Subcase:

$$
\mathcal{D}=\frac{\Delta ; \Gamma_{1}, A, \Gamma_{2} \Rightarrow C}{\Delta ; \Gamma_{1}, A, \Gamma_{2} \Rightarrow \diamond C} \diamond \mathrm{R}
$$

(1) $\Delta ; \Gamma_{1}, \Gamma_{2} \Rightarrow C$

(2) $\Delta ; \Gamma_{1}, \Gamma_{2} \Rightarrow \diamond C$

Subcase:

$$
\mathcal{D}=\frac{\Delta, B ; \Gamma_{1}, A, \Gamma_{2} \Rightarrow C}{\Delta ; \Gamma_{1}, A, \Gamma_{2} \Rightarrow C} \square \mathrm{L} \quad\left(\square B \in \Gamma_{1}, \Gamma_{2}\right)
$$

(1) $\Delta, B ; \Gamma_{1}, \Gamma_{2} \Rightarrow A$

Lemma $21(\mathcal{E})$

(2) $\Delta, B ; \Gamma_{1}, \Gamma_{2} \Rightarrow C$

i.h. $\left(\mathcal{D}_{1}, 1\right)$

(3) $\Delta ; \Gamma_{1}, \Gamma_{2} \Rightarrow C$

Subcase:

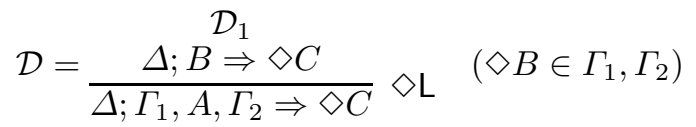

(1) $\Delta ; \Gamma_{1}, \Gamma_{2} \Rightarrow \diamond C$

Case: The cut formula is not the principal formula of the last inference of $\mathcal{E}$.

Subcase:

$$
\mathcal{E}=\frac{\mathcal{E}_{1}}{\Delta, B ; \Gamma_{1}, \Gamma_{2} \Rightarrow A} \quad \square \mathrm{L} \quad\left(\square B \in \Gamma_{1}, \Gamma_{2}\right)
$$

(1) $\Delta, B ; \Gamma_{1}, A, \Gamma_{2} \Rightarrow C$

Lemma $21(\mathcal{D})$

(2) $\Delta, B ; \Gamma_{1}, \Gamma_{2} \Rightarrow C$

i.h. $\left(1, \mathcal{E}_{1}\right)$

(3) $\Delta ; \Gamma_{1}, \Gamma_{2} \Rightarrow C$

Subcase:

$$
\mathcal{D}=\frac{\Delta ; A \stackrel{\mathcal{D}_{1}}{\Rightarrow} \diamond C}{\Delta ; \Gamma_{1}, \diamond A, \Gamma_{2} \Rightarrow \diamond C} \diamond \mathrm{L}
$$

and

$$
\mathcal{E}=\frac{\Delta ; B \Rightarrow \diamond A}{\Delta ; \Gamma_{1}, \Gamma_{2} \Rightarrow \diamond A} \diamond \mathrm{L} \quad\left(\diamond B \in \Gamma_{1}, \Gamma_{2}\right)
$$

Here, we require the last inference of $\mathcal{D}$ to have the cut formula $\diamond A$ as its principal formula. Our cases remain exhaustive since the cut formula not being the principal formula of the last inference of $\mathcal{D}$ is covered by the previous case.
(1) $\Delta ; \diamond A, B \Rightarrow \diamond C$
$\diamond \mathrm{L}\left(\mathcal{D}_{1}\right)$
(2) $\Delta ; B \Rightarrow \diamond C$
i.h. $\left(1, \mathcal{E}_{1}\right)$
(3) $\Delta ; \Gamma_{1}, \Gamma_{2} \Rightarrow \diamond C$
$\diamond \mathrm{L}(2)$ 
Next, we give the cases for modal cut. Here, we can appeal to the induction hypothesis for cut even if the size of the cut formula stays the same.

Case:

$$
\mathcal{F}=\overline{\Delta_{1}, A, \Delta_{2} ; \Gamma_{1}, C, \Gamma_{2} \Rightarrow C} \text { init }
$$

Case:

(1) $\Delta_{1}, \Delta_{2} ; \Gamma_{1}, C, \Gamma_{2} \Rightarrow C \quad$ init

\section{Case:}

$$
\mathcal{F}=\overline{\Delta_{1}, A, \Delta_{2} ; \Gamma_{1}, \perp, \Gamma_{2} \Rightarrow C} \perp \mathrm{L}
$$

(1) $\Delta_{1}, \Delta_{2} ; \Gamma_{1}, \perp, \Gamma_{2} \Rightarrow C$

$$
\mathcal{F}=\frac{\mathcal{F}_{1}, A, \Delta_{2} ; \Gamma, B \Rightarrow C}{\Delta_{1}, A, \Delta_{2} ; \Gamma \Rightarrow C} \text { refl } \quad\left(B \in \Delta_{1}, \Delta_{2}\right)
$$

(1) $\Delta_{1}, \Delta_{2} ; \Gamma, B \Rightarrow C$

(2) $\Delta_{1}, \Delta_{2} ; \Gamma \Rightarrow C$

i.h. $\left(\mathcal{F}_{1}, \mathcal{G}\right)$ refl (1)

Case:

$$
\mathcal{F}=\frac{\mathcal{F}_{1}}{\Delta_{1}, A, \Delta_{2} ; \Gamma, A \Rightarrow C} \text { refl }
$$
(1) $\Delta_{1}, \Delta_{2} ; \Gamma, A \Rightarrow C$
(2) $\Delta_{1}, \Delta_{2} ; \Gamma \Rightarrow A$
(3) $\Delta_{1}, \Delta_{2} ; \Gamma \Rightarrow C$

i.h. $\left(\mathcal{F}_{1}, \mathcal{G}\right)$

Lemma $20(\mathcal{G})$

i.h. $(1,2)$

\section{Case:}

$$
\mathcal{F}=\frac{\mathcal{F}_{1}, A, \Delta_{2} ; \cdot \Rightarrow C}{\Delta_{1}, A, \Delta_{2} ; \Gamma \Rightarrow \square C} \square \mathrm{R}
$$

(1) $\Delta_{1}, \Delta_{2} ; \cdot \Rightarrow C$

(2) $\Delta_{1}, \Delta_{2} ; \Gamma \Rightarrow \square C$

i.h. $\left(\mathcal{F}_{1}, \mathcal{G}\right)$

$\square \mathrm{R}(1)$

Case:

$$
\mathcal{F}=\frac{\Delta_{1}, A, \Delta_{2}, B ; \Gamma_{1}, \square B, \Gamma_{2} \Rightarrow C}{\Delta_{1}, A, \Delta_{2} ; \Gamma_{1}, \square B, \Gamma_{2} \Rightarrow C} \square \mathrm{L}
$$

(1) $\Delta_{1}, \Delta_{2}, B ; \cdot \Rightarrow A$

(2) $\Delta_{1}, \Delta_{2}, B ; \Gamma_{1}, \square B, \Gamma_{2} \Rightarrow C$

(3) $\Delta_{1}, \Delta_{2} ; \Gamma_{1}, \square B, \Gamma_{2} \Rightarrow C$

Lemma $21(\mathcal{G})$

i.h. $\left(\mathcal{F}_{1}, 1\right)$

$\square \mathrm{L}(2)$

Case:

$$
\mathcal{F}=\frac{\Delta_{1}, A, \mathcal{F}_{2} ; \Gamma \Rightarrow C}{\Delta_{1}, A, \Delta_{2} ; \Gamma \Rightarrow \diamond C} \diamond \mathrm{R}
$$

(1) $\Delta_{1}, \Delta_{2} ; \Gamma \Rightarrow C$

(2) $\Delta_{1}, \Delta_{2} ; \Gamma \Rightarrow \diamond C$

i.h. $\left(\mathcal{F}_{1}, \mathcal{G}\right)$ $\diamond \mathrm{R}(1)$

Case:

$$
\mathcal{F}=\frac{\Delta_{1}, A, \Delta_{2} ; B \Rightarrow \diamond C}{\Delta_{1}, A, \Delta_{2} ; \Gamma_{1}, \diamond B, \Gamma_{2} \Rightarrow \diamond C} \diamond \mathrm{L}
$$

(1) $\Delta_{1}, \Delta_{2} ; B \Rightarrow \diamond C$

(2) $\Delta_{1}, \Delta_{2} ; \Gamma_{1}, \diamond B, \Gamma_{2} \Rightarrow \diamond C$

i.h. $\left(\mathcal{F}_{1}, \mathcal{G}\right)$ $\diamond \mathrm{L}(1)$ 
Theorem 8 (Completeness of $\mathrm{LJ}_{\mathrm{IS} 4}$ with respect to $\mathrm{LJ}_{\mathrm{IS} 4}^{+}$). $\mathcal{D}$

If $\Delta ; \Gamma \stackrel{+}{\Rightarrow} A$, then $\Delta ; \Gamma \Rightarrow A$.

Proof. By induction on the structure of $\mathcal{D}$, using Lemma 33 where needed.

Theorem 9 (Completeness of $\mathrm{NJ}_{\mathrm{IS} 4}^{\mathrm{N}}$ with respect to $\mathrm{LJ}_{\mathrm{IS} 4}$ ). If $\Delta ; \Gamma \Rightarrow A$, then $\Delta ; \Gamma \vdash A \uparrow$.

Proof. By induction on the structure of $\mathcal{D}$. We show the base cases and the cases involving modalities.

Case:

$$
\mathcal{D}=\overline{\Delta ; \Gamma_{1}, A, \Gamma_{2} \Rightarrow A} \text { init }
$$

(1) $\Delta ; \Gamma_{1}, A, \Gamma_{2} \vdash A \downarrow$

hyp $_{1}$

(2) $\Delta ; \Gamma_{1}, A, \Gamma_{2} \vdash A \uparrow$

Case:

$$
\mathcal{D}=\overline{\Delta ; \Gamma_{1}, \perp, \Gamma_{2} \Rightarrow A} \perp \mathrm{L}
$$

(1) $\Delta ; \Gamma_{1}, \perp, \Gamma_{2} \vdash \perp \downarrow$

Case:

$$
\mathcal{D}=\frac{\mathcal{D}_{1}, B, \Delta_{2} ; \Gamma, B \Rightarrow A}{\Delta_{1}, B, \Delta_{2} ; \Gamma \Rightarrow A} \text { refl }
$$

(1) $\Delta_{1}, B, \Delta_{2} ; \Gamma, B \vdash A \uparrow$

(2) $\Delta_{1}, B, \Delta_{2} ; \Gamma \vdash B \downarrow$

i.h. $\left(\mathcal{D}_{1}\right)$ hyp $_{2}$

Case:

(3) $\Delta_{1}, B, \Delta_{2} ; \Gamma \vdash A \uparrow$

$$
\mathcal{D}=\frac{\Delta_{1}{ }^{\prime} \cdot \stackrel{\Rightarrow}{\Rightarrow} A}{\Delta ; \Gamma \Rightarrow \square A} \square \mathrm{R}
$$

(1) $\Delta ; \cdot \vdash A \uparrow$

(2) $\Delta ; \Gamma \vdash \square A \uparrow$

i.h. $\left(\mathcal{D}_{1}\right)$

Case:

$$
\mathcal{D}=\frac{\Delta, B ; \Gamma_{1}, \square B, \Gamma_{2} \Rightarrow A}{\square ; \Gamma_{1}, \square B, \Gamma_{2} \Rightarrow A} \square \mathrm{L}
$$
(1) $\Delta ; \Gamma_{1}, \square B, \Gamma_{2} \vdash \square B \downarrow$
(2) $\Delta, B ; \Gamma_{1}, \square B, \Gamma_{2} \vdash A \uparrow$
hyp $_{1}$
(3) $\Delta ; \Gamma_{1}, \square B, \Gamma_{2} \vdash A \uparrow$

Case:

$$
\mathcal{D}=\frac{\Delta ; \Gamma_{1} \Rightarrow A}{\Delta ; \Gamma \Rightarrow \diamond A} \diamond \mathrm{R}
$$
(1) $\Delta ; \Gamma \vdash A \uparrow$
(2) $\Delta ; \Gamma \vdash \diamond A \uparrow$

i.h. $\left(\mathcal{D}_{1}\right)$ $\diamond \mathbf{I}(1)$ 
Case:

$$
\mathcal{D}=\frac{\Delta ; B \Rightarrow \diamond A}{\Delta ; \Gamma_{1}, \diamond B, \Gamma_{2} \Rightarrow \diamond A} \diamond \mathrm{L}
$$

(1) $\Delta ; \Gamma_{1}, \diamond B, \Gamma_{2} \vdash \diamond B \downarrow$

hyp $_{1}$

(2) $\Delta ; B \vdash \diamond A \uparrow$

i.h. $\left(\mathcal{D}_{1}\right)$

(3) $\Delta ; \Gamma_{1}, \diamond B, \Gamma_{2} \vdash \diamond A \uparrow$

$\diamond \mathrm{E}(1,2)$

With this collection of intermediate results, the completeness of $\mathbf{N J}_{\mathbf{I S} 4}^{\mathbf{N}}$ with respect to $\mathbf{N J}_{\mathbf{I S} 4}$ follows in a step-by-step way.

Theorem 10 (Completeness of $\mathrm{NJ}_{\mathrm{IS} 4}^{\mathrm{N}}$ with respect to $\mathrm{NJ}_{\mathrm{IS} 4}$ ). If $\Delta ; \stackrel{\mathcal{D}}{\Gamma} A$, then $\Delta ; \Gamma \vdash A \uparrow$.

Proof.

(1) $\Delta ; \Gamma \vdash_{+} A \uparrow$

Theorem $6(\mathcal{D})$

(2) $\Delta ; \Gamma \stackrel{+}{\Rightarrow} A$

Theorem 7 (1)

(3) $\Delta ; \Gamma \Rightarrow A$

Theorem $8(2)$

(4) $\Delta ; \Gamma \vdash A \uparrow$

Theorem $9(3)$

Theorem 10 is a weak normalization result: if a judgement is derivable in our natural deduction system $\mathbf{N J}_{\mathbf{I S} 4}$, then it has a normal natural deduction, where the normal forms of natural deductions are defined by the restrictions imposed by the arrow annotations in $\mathbf{N J}_{\mathbf{I S} 4}^{\mathbf{N}}$. An alternative normalization proof is to explicitly consider the normalization steps - $\beta$-conversions and commuting (or permutative) conversions - arising from the rules of $\mathbf{N J}_{\mathbf{I S 4}}$, and to show that for any derivation, a sequence of normalization steps can be found that terminates in a corresponding derivation in normal form. What then remains to be shown is that a derivation can be constructed in $\mathbf{N} \mathbf{J}_{\mathbf{I S} 4}^{\mathbf{N}}$ if and only if it is in normal form. We will not give the details of such an alternative proof, but the conversions arising from the $\mathbf{N} \mathbf{J}_{\text {IS4 }}$ rules involving modalities are as follows, while those for the remaing rules are standard (see for instance [10] or [21]).

1. $\beta$-conversion for $\square \mathrm{l}$ and $\square \mathrm{E}$ :

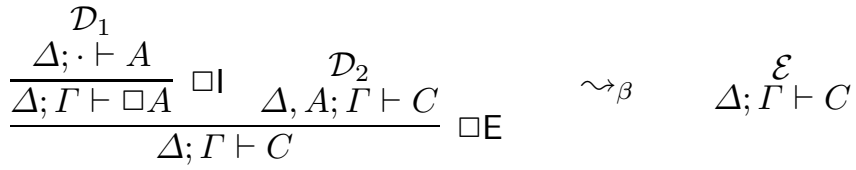

where $\mathcal{E}$ is obtained by applying Lemma 6 to $\mathcal{D}_{2}$ and $\mathcal{D}_{1}$.

2. $\beta$-conversion for $\diamond \mathrm{I}$ and $\diamond \mathrm{E}$ :

$$
\begin{aligned}
& \mathcal{D}_{1}
\end{aligned}
$$

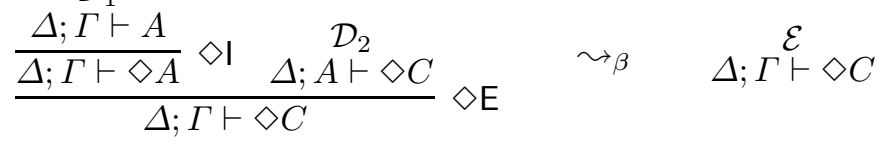


where $\mathcal{E}$ is obtained by applying Lemma 1 to $\mathcal{D}_{2}$ to obtain $\Delta ; \Gamma, A \vdash \diamond C$ and then applying Lemma 5 to this and $\mathcal{D}_{1}$.

3. Commuting conversion for $\square \mathrm{E}$ :

$$
\begin{aligned}
& \begin{array}{cc}
\mathcal{D}_{1} & \mathcal{D}_{2}
\end{array}
\end{aligned}
$$

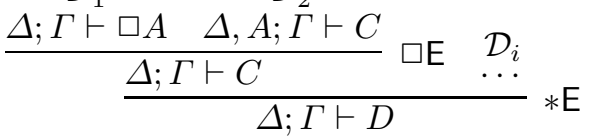

$$
\begin{aligned}
& \sim_{c} \begin{array}{cc}
\mathcal{D}_{2} & \mathcal{E}_{i} \\
\mathcal{D}_{1} & \frac{\Delta, A ; \Gamma \vdash C \quad \cdots}{\Delta, A ; \Gamma \vdash D} \\
\frac{\Delta ; \Gamma \vdash \square}{\Delta ; \Gamma \vdash D}
\end{array} \mathrm{E}
\end{aligned}
$$

where $* \mathrm{E}$ is any elimination rule, $\cdots$ denotes some number of minor premises with derivations $\mathcal{D}_{i}, i=3,4, \ldots$, and $\mathcal{E}_{i}$ is obtained by applying Lemma 2 to $\mathcal{D}_{i}$ to add $A$ to the modal context of the final judgement.

4. Commuting conversion for $\diamond \mathrm{E}$ :

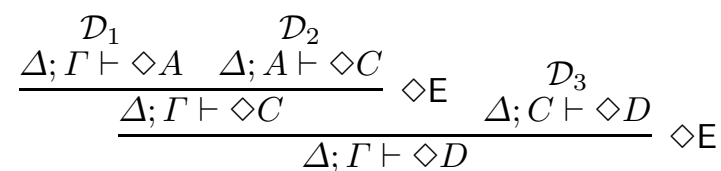

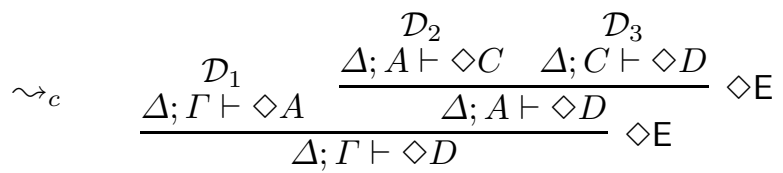

Since the succedent of the conclusion of an application of $\diamond E$ is of the form $\diamond C$ and the only elimination rule whose major premise has a succedent of the form $\diamond C$ is $\diamond \mathrm{E}$, this is the only commuting conversion arising from the $\diamond$ modality.

As a platform for proof search, $\mathbf{N J}_{\mathbf{I S} 4}^{\mathbf{N}}$ is superior to $\mathbf{N J}_{\mathbf{I S} 4}$, since the proof space is restricted to allow only normal natural deductions. However, $\mathbf{N} \mathbf{J}_{\mathbf{I S} 4}^{\mathbf{N}}$ still suffers from a lack of syntax-directedness in the case of the elimination rules, an issue that we will address by using techniques from forward proof search. Although we will ultimately return to $\mathbf{N J}_{\mathbf{I S} 4}^{\mathbf{N}}$ in our search for bidirectional decision procedures, the relationship between introduction and elimination rules and backward and forward proof search is perhaps most vividly demonstrated in a focused sequent calculus setting, which we turn to next.

\section{Sequent Calculi}

Although we could use $\mathbf{L} \mathbf{J}_{\mathbf{I S} 4}$, presented in Sect. 4, as a basis for proof search in a backward direction, its refl rule is a source of inconvenient nondeterminism. We will instead follow the approach of Dyckhoff and Pinto [6] and construct a 


$$
\begin{aligned}
& \overline{\Delta ; \Gamma \triangleright A \rightarrow A} \text { init } \quad(A \text { is atomic) } \quad \overline{\Delta ; \Gamma \triangleright \perp \rightarrow A} \perp \mathrm{L} \\
& \frac{\Delta ; \Gamma_{1}, A, \Gamma_{2} \triangleright A \rightarrow C}{\Delta ; \Gamma_{1}, A, \Gamma_{2} \rightarrow C} \operatorname{ch}_{1} \frac{\Delta_{1}, A, \Delta_{2} ; \Gamma \triangleright A \rightarrow C}{\Delta_{1}, A, \Delta_{2} ; \Gamma \rightarrow C} \mathrm{ch}_{2} \\
& \frac{\Delta ; \Gamma, A_{1} \rightarrow A_{2}}{\Delta ; \Gamma \rightarrow A_{1} \supset A_{2}} \supset \mathrm{R} \quad \frac{\Delta ; \Gamma \rightarrow A_{1} \quad \Delta ; \Gamma \triangleright A_{2} \rightarrow C}{\Delta ; \Gamma \triangleright A_{1} \supset A_{2} \rightarrow C} \supset \mathrm{L} \\
& \frac{\Delta ; \Gamma \rightarrow A_{1} \quad \Delta ; \Gamma \rightarrow A_{2}}{\Delta ; \Gamma \rightarrow A_{1} \wedge A_{2}} \wedge \mathrm{R} \frac{\Delta ; \Gamma \triangleright A_{j} \rightarrow C}{\Delta ; \Gamma \triangleright A_{1} \wedge A_{2} \rightarrow C} \wedge \mathrm{L}_{j} \\
& \frac{\Delta ; \Gamma \rightarrow A_{j}}{\Delta ; \Gamma \rightarrow A_{1} \vee A_{2}} \vee \mathrm{R}_{j} \quad \frac{\Delta ; \Gamma, A_{1} \rightarrow C \quad \Delta ; \Gamma, A_{2} \rightarrow C}{\Delta ; \Gamma \triangleright A_{1} \vee A_{2} \rightarrow C} \vee \mathrm{L} \\
& \frac{\Delta ; \cdot \rightarrow A}{\Delta ; \Gamma \rightarrow \square A} \square \mathrm{R} \quad \frac{\Delta, A ; \Gamma \rightarrow C}{\Delta ; \Gamma \triangleright \square A \rightarrow C} \square \mathrm{L} \\
& \frac{\Delta ; \Gamma \rightarrow A}{\Delta ; \Gamma \rightarrow \diamond A} \diamond \mathrm{R} \quad \frac{\Delta ; A \rightarrow \diamond C}{\Delta ; \Gamma \triangleright \diamond A \rightarrow \diamond C} \diamond \mathrm{L} \\
& j \in\{1,2\}
\end{aligned}
$$

Fig. 5. $\mathrm{MJ}_{\mathrm{IS} 4}$

focused sequent calculus for propositional IS4 whose derivations are in bijective correspondence with normal natural deductions. This system, which we will call $\mathbf{M J}_{\mathbf{I S} 4}$, is shown in Fig. 5 and involves two forms of sequents:

$$
\begin{array}{ll}
\Delta ; \Gamma \rightarrow C & C \text { can be proved from assumptions } \Delta, \Gamma, \\
\Delta ; \Gamma \triangleright A \rightarrow C & C \text { can be proved from assumptions } \Delta, \Gamma, A, \text { focusing on } \\
& \text { the assumption } A .
\end{array}
$$

If a sequent is focused on a formula $A$, then the only applicable rules are those with $A$ as a principal formula. Following Girard [9], we will call the position of the focused formula the stoup. As in the natural deduction formulations, contexts in $\mathbf{M J}_{\mathbf{I S} 4}$ are technically ordered lists, but the usual structural properties of weakening, contraction, and exchange hold here as well, so an interpretation of contexts as multisets is reasonable.

\section{Lemma 34 (Weakening for $\mathrm{MJ}_{\mathrm{IS} 4}$ ).}
$\mathcal{D}$
1. If $\Delta ; \Gamma_{1}, \Gamma_{2} \rightarrow C$, then $\Delta ; \Gamma_{1}, A, \Gamma_{2} \rightarrow C$.
2. If $\Delta ; \Gamma_{1}, \Gamma_{2} \triangleright B \rightarrow C$, then $\Delta ; \Gamma_{1}, A, \Gamma_{2} \triangleright B \rightarrow C$.

Proof. By simultaneous induction on the structure of the given derivation.

\section{Lemma 35 (Modal weakening for $\mathrm{MJ}_{\mathrm{IS} 4}$ ).}

1. If $\Delta_{1}, \stackrel{\mathcal{D}}{,} \Delta_{2} ; \Gamma \rightarrow C$, then $\Delta_{1}, A, \Delta_{2} ; \Gamma \rightarrow C$. 
2. If $\Delta_{1}, \Delta_{2} ; \Gamma \triangleright B \rightarrow C$, then $\Delta_{1}, A, \Delta_{2} ; \Gamma \triangleright B \rightarrow C$.

Proof. By simultaneous induction on the structure of the given derivation.

Lemma 36 (Contraction for $\mathrm{MJ}_{\mathrm{IS} 4}$ ).
$\mathcal{D}$
1. If $\Delta ; \Gamma_{1}, A, \Gamma_{2}, A, \Gamma_{3} \rightarrow C$, then $\Delta ; \Gamma_{1}, A, \Gamma_{2}, \Gamma_{3} \rightarrow C$.
2. If $\Delta ; \Gamma_{1}, A, \Gamma_{2}, \mathcal{E} A, \Gamma_{3} \triangleright B \rightarrow C$, then $\Delta ; \Gamma_{1}, A, \Gamma_{2}, \Gamma_{3} \triangleright B \rightarrow C$.

Proof. By simultaneous induction on the structure of the given derivation.

Lemma 37 (Modal contraction for $\mathrm{MJ}_{\mathrm{IS} 4}$ ).
1. If $\Delta_{1}, A, \Delta_{2}, \stackrel{\mathcal{D}}{A, \Delta_{3}} ; \Gamma \rightarrow C$, then $\Delta_{1}, A, \Delta_{2}, \Delta_{3} ; \Gamma \rightarrow C$.
2. If $\Delta_{1}, A, \Delta_{2}, A, \Delta_{3} ; \Gamma \triangleright B \rightarrow C$, then $\Delta_{1}, A, \Delta_{2}, \Delta_{3} ; \Gamma \triangleright B \rightarrow C$.

Proof. By simultaneous induction on the structure of the given derivation.

\section{Lemma 38 (Spanning contraction for $\mathrm{MJ}_{\mathrm{IS} 4}$ ).}
1. If $\Delta_{1}, A, \Delta_{2} ; \Gamma_{1}, \underset{\mathcal{E}}{A}, \Gamma_{2} \rightarrow C$, then $\Delta_{1}, A, \Delta_{2} ; \Gamma_{1}, \Gamma_{2} \rightarrow C$.
2. If $\Delta_{1}, A, \Delta_{2} ; \Gamma_{1}, A, \Gamma_{2} \triangleright B \rightarrow C$, then $\Delta_{1}, A, \Delta_{2} ; \Gamma_{1}, \Gamma_{2} \triangleright B \rightarrow C$.

Proof. By simultaneous induction on the structure of the given derivation, replacing applications of $\mathrm{ch}_{1}$ by applications of $\mathrm{ch}_{2}$ where needed.

The key result of this section is that there is a very close correspondence between $\mathbf{M} \mathbf{J}_{\mathbf{I S} 4}$ and $\mathbf{N J}_{\mathbf{I S} 4}^{\mathbf{N}}$. In fact, derivations in one correspond bijectively to derivations in the other. To show this, we will define functions that map $\mathbf{M} \mathbf{J}_{\mathbf{I S} 4}$ derivations to corresponding $\mathbf{N J}_{\mathbf{I S} 4}^{\mathbf{N}}$ derivations and vice versa.

More formally, we define a function $f$ that takes an $\mathbf{M} \mathbf{J}_{\text {IS4 }}$ derivation of $\Delta ; \Gamma \rightarrow C$ and returns a corresponding $\mathbf{N J}_{\mathbf{I S} 4}^{\mathbf{N}}$ derivation of $\Delta ; \Gamma \vdash C \uparrow$. It requires an auxiliary function $f^{\prime}$ which takes two arguments, an $\mathbf{M} \mathbf{J}_{\text {IS4 }}$ derivation of $\Delta ; \Gamma \triangleright A \rightarrow C$ and an $\mathbf{N} \mathbf{J}_{\mathbf{I S} 4}^{\mathbf{N}}$ derivation of $\Delta ; \Gamma \vdash A \downarrow$. Given these arguments, $f^{\prime}$ returns an $\mathbf{N J}_{\mathbf{I S} 4}^{\mathbf{N}}$ derivation of $\Delta ; \Gamma \vdash C \uparrow$. The operational interpretation is that $f$ is the main generator function, traversing the unfocused segments of the given $\mathbf{M J}_{\mathbf{I S} 4}$ derivation in an upward direction, producing corresponding segments of $\uparrow$ judgements of a partial $\mathbf{N J}_{\mathbf{I S} 4}^{\mathbf{N}}$ derivation, also constructed in an upward direction. When a formula is raised into the stoup in the $\mathbf{M} \mathbf{J}_{\mathbf{I S} 4}$ derivation being traversed, the auxiliary function $f^{\prime}$ is called upon to continue traversing the $\mathbf{M J}_{\mathbf{I S} 4}$ derivation (the first argument to $f^{\prime}$ ) in an upward direction, while the corresponding segment of $\downarrow$ judgements in the partial $\mathbf{N} \mathbf{J}_{\text {IS4 }}^{\mathbf{N}}$ derivation (the second argument to $f^{\prime}$ ) is constructed in a downward direction. When the focus in the original $\mathbf{M} \mathbf{J}_{\mathbf{I S} 4}$ derivation blurs, the downward-constructed partial $\mathbf{N J}_{\mathbf{I S} \text { 4 }}^{\mathbf{N}}$ derivation meets the previously upward-constructed partial $\mathbf{N J}_{\mathbf{I S} 4}^{\mathbf{N}}$ derivation, 
and the main function $f$ is possibly called upon again to continue the traversal of the next unfocused segment of the $\mathbf{M} \mathbf{J}_{\mathbf{I S} 4}$ derivation.

The function $f$ is defined as follows.

$$
\begin{aligned}
& f\left(\frac{\Delta ; \Gamma_{1}, A, \Gamma_{2} \triangleright A \rightarrow C}{\Delta ; \Gamma_{1}, A, \Gamma_{2} \rightarrow C} \mathrm{ch}_{1}\right) \stackrel{\text { def }}{=} f^{\prime}\left(\mathcal{D}_{1}, \frac{}{\Delta ; \Gamma_{1}, A, \Gamma_{2} \vdash A \downarrow} \text { hyp }_{1}\right) \\
& f\left(\frac{\Delta_{1}, A, \Delta_{2} ; \Gamma \triangleright A \rightarrow C}{\Delta_{1}, A, \Delta_{2} ; \Gamma \rightarrow C} \mathrm{ch}_{2}\right) \stackrel{\text { def }}{=} f^{\prime}\left(\mathcal{D}_{1}, \frac{}{\Delta_{1}, A, \Delta_{2} ; \Gamma \vdash A \downarrow} \text { hyp }_{2}\right) \\
& f\left(\frac{\Delta ; \Gamma, A_{1} \rightarrow A_{2}}{\Delta ; \Gamma \rightarrow A_{1} \supset A_{2}} \supset \mathrm{R}\right) \stackrel{\text { def }}{=} \frac{\begin{array}{c}
f\left(\mathcal{D}_{1}\right) \\
\Delta ; \Gamma, A_{1} \vdash A_{2} \uparrow
\end{array}}{\Delta ; \Gamma \vdash A_{1} \supset A_{2} \uparrow} \supset \mathrm{l}
\end{aligned}
$$

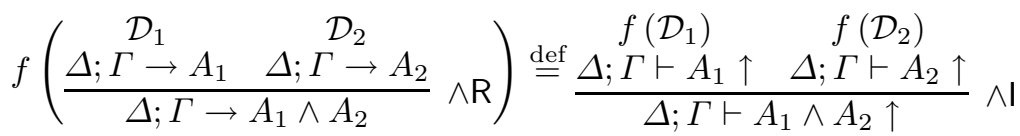

$$
\begin{aligned}
& f\left(\frac{\Delta ; \Gamma \rightarrow A_{j}}{\Delta ; \Gamma \rightarrow A_{1} \vee A_{2}} \vee \mathrm{R}_{j}\right) \stackrel{\mathcal{D}_{1}}{=} \frac{\begin{array}{c}
f\left(\mathcal{D}_{1}\right) \\
\Delta ; \Gamma \vdash A_{j} \uparrow
\end{array}}{\Delta ; \Gamma A_{1} \vee A_{2} \uparrow} \vee \mathrm{I}_{j} \\
& f\left(\begin{array}{c}
\mathcal{D}_{1} ; \cdot \rightarrow A \\
\Delta ; \Gamma \rightarrow \square A \\
\Delta \mathrm{R}
\end{array}\right) \stackrel{\text { def }}{=} \frac{f\left(\mathcal{D}_{1}\right)}{\Delta ; \cdot \vdash A \uparrow}
\end{aligned}
$$

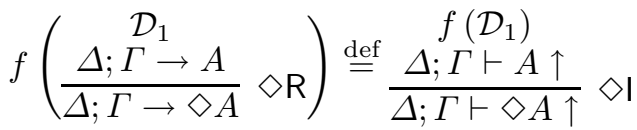

$$
\begin{aligned}
& j \in\{1,2\}
\end{aligned}
$$

The function $f^{\prime}$ is defined as follows.

$$
\begin{aligned}
& f^{\prime}(\overline{\Delta ; \Gamma \triangleright A \rightarrow A} \text { init, } \mathcal{F}) \stackrel{\text { def }}{=} \frac{\Delta ; \Gamma \vdash A \downarrow}{\Delta ; \Gamma \vdash A \uparrow} \uparrow \downarrow \\
& f^{\prime}(\overline{\Delta ; \Gamma \triangleright \perp \rightarrow A} \perp \mathrm{L}, \mathcal{F}) \stackrel{\text { def }}{=} \frac{\Delta ; \Gamma \vdash \perp \downarrow}{\Delta ; \Gamma \vdash A \uparrow} \perp \mathrm{E} \\
& f^{\prime}\left(\begin{array}{c}
\mathcal{E}_{1} \\
\frac{\Delta, A ; \Gamma \rightarrow C}{\Delta ; \Gamma \triangleright \square A \rightarrow C} \square \mathrm{L}^{\prime}
\end{array}, \stackrel{\mathcal{F}}{\stackrel{\text { def }}{=}} \frac{\Delta ; \Gamma \stackrel{\mathcal{F}}{\vdash} \square A \downarrow \quad \Delta, A ; \Gamma \vdash C \uparrow}{\Delta ; \Gamma \vdash C \uparrow} \square \mathrm{E}\right. \\
& f^{\prime}\left(\frac{\Delta ; A \rightarrow \diamond C}{\Delta ; \Gamma \triangleright \diamond A \rightarrow \diamond C} \diamond \mathrm{L}^{\prime}, \mathcal{F}\right) \stackrel{\text { def }}{=} \frac{\Delta ; \Gamma \vdash \diamond A \downarrow \quad \Delta ; A \vdash \diamond C \uparrow}{\Delta ; \Gamma \vdash \diamond C \uparrow} \diamond \mathrm{E}
\end{aligned}
$$

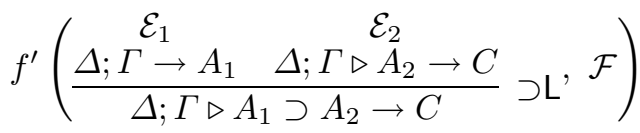




$$
\begin{aligned}
& \stackrel{\text { def }}{=} f^{\prime}\left(\mathcal{E}_{2}, \frac{\left.\Delta ; \Gamma \vdash \stackrel{\mathcal{F}}{A_{1} \supset A_{2} \downarrow} \Delta ; \mathcal{E}_{1}\right)}{\Delta ; \Gamma \vdash A_{2} \downarrow} \supset \mathrm{E}\right) \\
& f^{\prime}\left(\frac{\Delta ; \Gamma \triangleright A_{j} \rightarrow C}{\Delta ; \Gamma \triangleright A_{1} \wedge A_{2} \rightarrow C} \wedge \mathrm{L}_{j}, \mathcal{F}\right) \\
& \stackrel{\text { def }}{=} f^{\prime}\left(\mathcal{E}_{1}, \frac{\Delta ; \Gamma \vdash \stackrel{\mathcal{F}}{A_{1} \wedge A_{2} \downarrow}}{\Delta ; \Gamma \vdash A_{j} \downarrow} \wedge \mathrm{E}_{j}\right)
\end{aligned}
$$

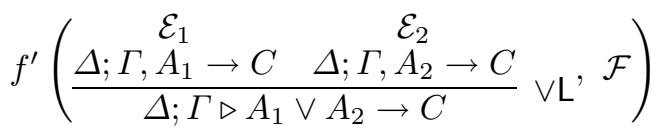

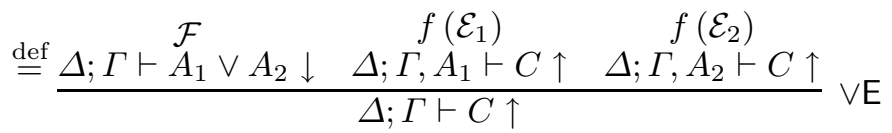

$$
\begin{aligned}
& j \in\{1,2\}
\end{aligned}
$$

The functions $g$ and $g^{\prime}$ are similar to $f$ and $f^{\prime}$, but convert $\mathbf{N} \mathbf{J}_{\mathbf{I S} 4}^{\mathbf{N}}$ derivations into $\mathbf{M} \mathbf{J}_{\mathbf{I S} 4}$ derivations. Formally, $g$ takes an $\mathbf{N J}_{\mathbf{I S} 4}^{\mathbf{N}}$ derivation of $\Delta ; \Gamma \vdash C \uparrow$ and returns a corresponding $\mathbf{M J}_{\mathbf{I S} 4}$ derivation of $\Delta ; \Gamma \rightarrow C$. It requires an auxiliary function $g^{\prime}$ which takes two arguments, an $\mathbf{N J}_{\mathbf{I S} 4}^{\mathbf{N}}$ derivation of $\Delta ; \Gamma \vdash A \downarrow$ and an $\mathbf{M J}_{\mathbf{I S} 4}$ derivation of $\Delta ; \Gamma \triangleright A \rightarrow C$. Given these arguments, $g^{\prime}$ returns an $\mathbf{M J}_{\mathbf{I S} 4}$ derivation of $\Delta ; \Gamma \rightarrow C$. The interactions between $g$ and $g^{\prime}$ are very similar to those between $f$ and $f^{\prime} . g$ is the main generator function that proceeds upward along the $\uparrow$ sequents of the given $\mathbf{N J}_{\mathbf{I S} 4}^{\mathbf{N}}$ derivation. The corresponding $\mathbf{M J}_{\mathbf{I S 4}}$ derivation is also constructed in an upward direction. If the direction of reasoning in the original $\mathbf{N} \mathbf{J}_{\mathbf{I S} 4}^{\mathbf{N}}$ derivation reverses, $g^{\prime}$ is called upon. Since the corresponding segment of the derivation in $\mathbf{M J}_{\mathbf{I S} 4}$ is focused, the construction of this focused $\mathbf{M} \mathbf{J}_{\mathbf{I S} 4}$ segment (the second argument to $g^{\prime}$ ) proceeds in a downward direction, while $g^{\prime}$ continues to traverse the original $\mathbf{N J}_{\mathbf{I S} 4}^{\mathbf{N}}$ derivation (the first argument to $g^{\prime}$ ) in an upward direction. The partial $\mathbf{M} \mathbf{J}_{\text {IS4 }}$ derivations meet when the leaves of the $\mathbf{N J}_{\mathbf{I S} 4}^{\mathbf{N}}$ derivation are reached.

The function $g$ is defined as follows.

$$
\begin{aligned}
& g\left(\frac{\Delta ; \Gamma \vdash A \downarrow}{\mathcal{D}_{1}} \underset{\Delta ; \Gamma \vdash A \uparrow}{ } \uparrow\right) \stackrel{\text { def }}{=} g^{\prime}\left(\mathcal{D}_{1}, \overline{\Delta ; \Gamma \triangleright A \rightarrow A} \text { init }\right) \\
& g\left(\frac{\Delta ; \Gamma \vdash \perp \downarrow}{\Delta ; \Gamma \vdash A \uparrow \perp \mathrm{E}}\right) \stackrel{\mathcal{D}_{1}}{=} g^{\prime}\left(\mathcal{D}_{1}, \overline{\Delta ; \Gamma \triangleright \perp \rightarrow A} \perp \mathrm{L}\right) \\
& g\left(\begin{array}{c}
\mathcal{D}_{1} \\
\frac{\Delta ; \Gamma, A_{1} \vdash A_{2} \uparrow}{\Delta ; \Gamma \vdash A_{1} \supset A_{2} \uparrow} \supset \mathrm{I}
\end{array}\right) \stackrel{\text { def }}{=} \frac{\begin{array}{c}
g\left(\mathcal{D}_{1}\right) \\
\Delta ; \Gamma, A_{1} \rightarrow A_{2}
\end{array}}{\Delta ; \Gamma \rightarrow A_{1} \supset A_{2}} \supset \mathrm{R}
\end{aligned}
$$




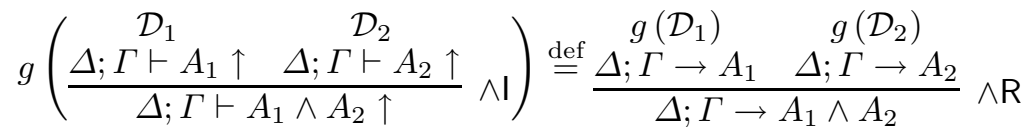

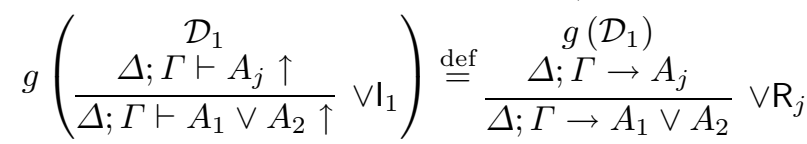

$$
\begin{aligned}
& g\left(\begin{array}{c}
\mathcal{D}_{1} ; \cdot \vdash A \uparrow \\
\Delta ; \Gamma \vdash \square A \uparrow \\
\Delta ;
\end{array}\right) \stackrel{\text { def }}{=} \frac{g\left(\mathcal{D}_{1}\right)}{\Delta ; \cdot \rightarrow A}
\end{aligned}
$$

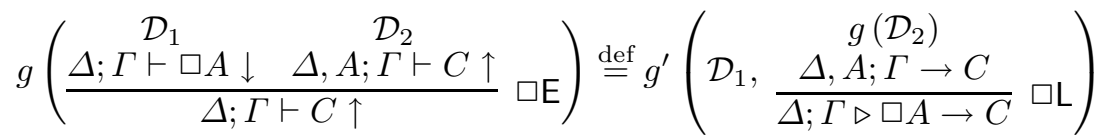

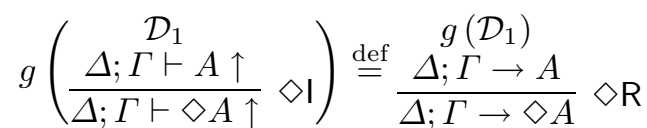

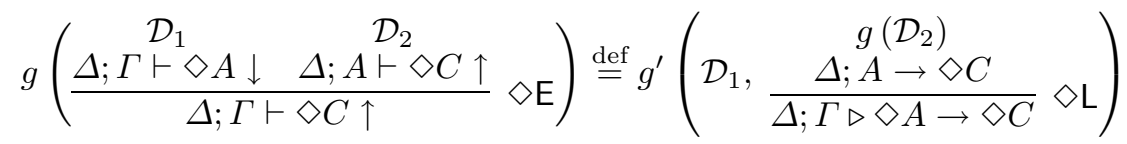

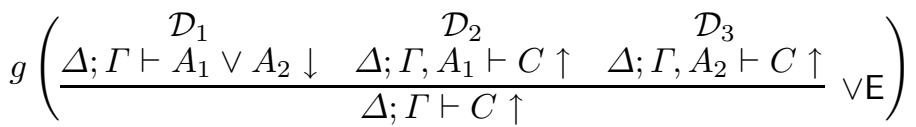

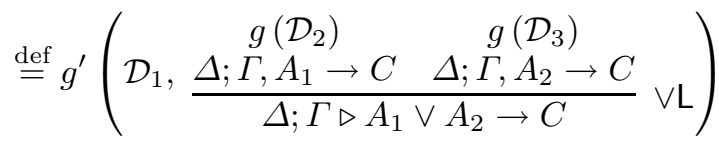

$$
\begin{aligned}
& j \in\{1,2\}
\end{aligned}
$$

The function $g^{\prime}$ is defined as follows.

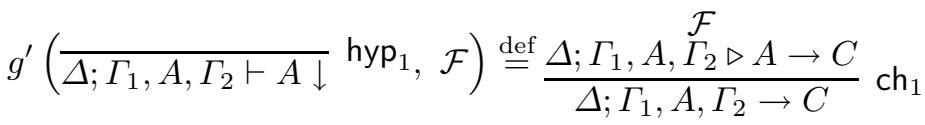

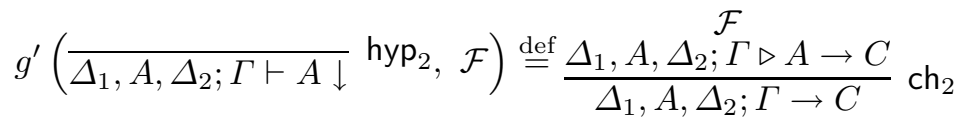

$$
\begin{aligned}
& g^{\prime}\left(\frac{\Delta ; \Gamma \vdash A_{1} \wedge A_{2} \downarrow}{\Delta ; \Gamma \vdash A_{j} \downarrow} \wedge \mathrm{E}_{j}, \mathcal{F}\right) \stackrel{\text { def }}{=} g^{\prime}\left(\mathcal{E}_{1}, \frac{\Delta ; \Gamma \triangleright A_{j} \rightarrow C}{\Delta ; \Gamma \triangleright A_{1} \wedge A_{2} \rightarrow C} \wedge \mathrm{L}_{j}\right) \\
& g^{\prime}\left(\frac{\Delta ; \Gamma \vdash A_{1} \supset A_{2} \downarrow \Delta ; \Gamma \vdash A_{1} \uparrow}{\Delta ; \Gamma \vdash A_{2} \downarrow} \supset \mathrm{E}, \mathcal{F}\right) \\
& \stackrel{\text { def }}{=} g^{\prime}\left(\begin{array}{cc}
g\left(\mathcal{E}_{2}\right) & \mathcal{F} \\
\mathcal{E}_{1}, \frac{\Delta ; \Gamma \stackrel{\mathcal{F}}{\rightarrow} A_{1} \quad \Delta ; \Gamma \triangleright A_{2} \rightarrow C}{\Delta ; \Gamma \triangleright A_{1} \supset A_{2} \rightarrow C} \supset \mathrm{L}
\end{array}\right)
\end{aligned}
$$




$$
j \in\{1,2\}
$$

Note that operationally, each call to any one of the functions $f, f^{\prime}, g$, or $g^{\prime}$ terminates, since the size of the first argument decreases in every recursive call. Finally, to establish a bijection between $\mathbf{M} \mathbf{J}_{\mathbf{I S} 4}$ and $\mathbf{N} \mathbf{J}_{\mathbf{I S} 4}^{\mathbf{N}}$ derivations, it is sufficient to show that $f$ and $g$ are both invertible. In fact, it is perhaps not surprising that $g$ is the inverse of $f$ and vice versa. The following two results formalize this notion.

Lemma 39 (Invertibility of $f$ and $g(1)$ ).

1. If $\Delta ; \stackrel{\mathcal{D}}{\vdash} C \uparrow$, then $f(g(\mathcal{D}))=\mathcal{D}$.

2. If $\Delta ; \Gamma \stackrel{\mathcal{E}}{\vdash} A \downarrow$ and $\Delta ; \Gamma \triangleright \mathcal{F} A \rightarrow C$, then $f\left(g^{\prime}(\mathcal{E}, \mathcal{F})\right)=f^{\prime}(\mathcal{F}, \mathcal{E})$.

Proof. By simultaneous induction on the structure of the first given derivation. We show the base cases and the cases involving modalities.

Case:

$$
\begin{aligned}
f\left(g^{\prime}\left(\frac{\mathcal{F}}{\Delta ; \Gamma_{1}, A, \Gamma_{2} \vdash A \downarrow} \operatorname{hyp}_{1}, \mathcal{F}\right)\right) & =f\left(\frac{\Delta ; \Gamma_{1}, A, \Gamma_{2} \triangleright A \rightarrow C}{\Delta ; \Gamma_{1}, A, \Gamma_{2} \rightarrow C} \mathrm{ch}_{1}\right) \\
& =f^{\prime}\left(\mathcal{F}, \overline{\Delta ; \Gamma_{1}, A, \Gamma_{2} \rightarrow A} \text { hyp }_{1}\right)
\end{aligned}
$$

Case:

$$
\begin{aligned}
f\left(g^{\prime}\left(\frac{\mathcal{F}}{\Delta_{1}, A, \Delta_{2} ; \Gamma \vdash A \downarrow} \operatorname{hyp}_{2}, \mathcal{F}\right)\right) & =f\left(\frac{\Delta_{1}, A, \Delta_{2} ; \Gamma \triangleright A \rightarrow C}{\Delta_{1}, A, \Delta_{2} ; \Gamma \rightarrow C} \mathrm{ch}_{2}\right) \\
& =f^{\prime}\left(\mathcal{F}, \overline{\Delta_{1}, A, \Delta_{2} ; \Gamma \rightarrow A} \text { hyp }_{2}\right)
\end{aligned}
$$

Case:

$$
\begin{aligned}
f\left(g\left(\frac{\Delta ; \Gamma \vdash \perp \downarrow}{\Delta ; \Gamma \vdash A \uparrow} \perp \mathrm{E}\right)\right) & =f\left(g^{\prime}\left(\mathcal{D}_{1}, \overline{\Delta ; \Gamma \triangleright \perp \rightarrow A} \perp \mathrm{L}\right)\right) \\
& \stackrel{\text { i.h }}{=} f^{\prime}\left(\overline{\Delta ; \Gamma \triangleright \perp \rightarrow A} \perp \mathrm{L}, \mathcal{D}_{1}\right) \\
& =\frac{\Delta ; \Gamma \vdash \perp \downarrow}{\Delta ; \Gamma \vdash A \uparrow} \perp \mathrm{E}
\end{aligned}
$$

\section{Case:}

$$
f\left(g\left(\begin{array}{c}
\mathcal{D}_{1} ; \cdot \vdash A \uparrow \\
\Delta ; \Gamma \vdash \square A \uparrow \\
\square ; \vdash
\end{array}\right)\right)=f\left(\begin{array}{c}
g\left(\mathcal{D}_{1}\right) \\
\frac{\Delta ; \cdot \rightarrow A}{\Delta ; \Gamma \rightarrow \square A} \square \mathrm{R}
\end{array}\right)
$$




$$
\begin{gathered}
f\left(g\left(\mathcal{D}_{1}\right)\right) \\
=\frac{\Delta ; \cdot \vdash A \uparrow}{\Delta ; \Gamma \vdash \square A \uparrow} \square \mathrm{I} \\
\stackrel{\mathcal{D}_{1}}{=} \frac{\Delta ; \cdot \vdash A \uparrow}{\Delta ; \Gamma \vdash \square A \uparrow} \square \mathrm{I}
\end{gathered}
$$

\section{Case:}

$$
\begin{aligned}
& f\left(g\left(\frac{\mathcal{D}_{1}}{\Delta ; \Gamma \vdash \square A \downarrow \quad \Delta, A ; \Gamma \vdash C \uparrow} \begin{array}{c}
\mathcal{D}_{2} \\
\Delta ; \Gamma \vdash C \uparrow
\end{array}\right)\right)
\end{aligned}
$$

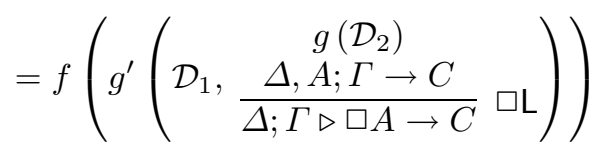

$$
\begin{aligned}
& \stackrel{\text { i.h. }}{=} f^{\prime}\left(\begin{array}{c}
g\left(\mathcal{D}_{2}\right) \\
\frac{\Delta, A ; \Gamma \rightarrow C}{\Delta ; \Gamma \triangleright \square A \rightarrow C} \square \mathrm{L},
\end{array}\right. \\
& \mathcal{D}_{1} \quad f\left(g\left(\mathcal{D}_{2}\right)\right) \\
& =\frac{\Delta ; \Gamma \vdash \square A \downarrow \quad \Delta, A ; \Gamma \vdash C \uparrow}{\Delta ; \Gamma \vdash C \uparrow} \square \mathrm{E}
\end{aligned}
$$

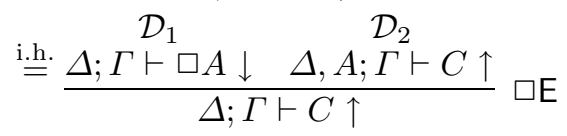

\section{Case:}

$$
\begin{aligned}
& f\left(g\left(\frac{\Delta ; \Gamma \vdash A \uparrow}{\mathcal{D}_{1}} \frac{\Delta ; \Gamma \vdash \diamond A \uparrow}{\Delta ; \mathrm{I}}\right)\right)=f\left(\begin{array}{c}
g\left(\mathcal{D}_{1}\right) \\
\frac{\Delta ; \Gamma \rightarrow A}{\Delta ; \Gamma \rightarrow \diamond A} \diamond \mathrm{R}
\end{array}\right) \\
& \begin{aligned}
& f\left(g\left(\mathcal{D}_{1}\right)\right) \\
= & \frac{\Delta ; \Gamma \vdash A \uparrow}{\Delta ; \Gamma \vdash \diamond A \uparrow} \diamond \mathrm{I}
\end{aligned} \\
& \stackrel{\text { i.h. }}{=} \frac{\mathcal{D}_{1}}{\Delta ; \Gamma \vdash A \uparrow}
\end{aligned}
$$

\section{Case:}

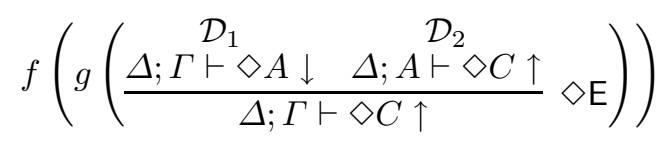




$$
\begin{aligned}
& =f\left(g^{\prime}\left(\begin{array}{c}
g\left(\mathcal{D}_{2}\right) \\
\mathcal{D}_{1}, \frac{\Delta ; A \rightarrow \diamond C}{\Delta ; \Gamma \triangleright \diamond A \rightarrow \diamond C} \diamond \mathrm{L}
\end{array}\right)\right) \\
& \stackrel{\text { i.h. }}{=} f^{\prime}\left(\begin{array}{c}
g\left(\mathcal{D}_{2}\right) \\
\frac{\Delta ; A \rightarrow \diamond C}{\Delta ; \Gamma \triangleright \diamond A \rightarrow \diamond C} \diamond \mathrm{L}^{\prime} \mathcal{D}_{1}
\end{array}\right) \\
& =\frac{\Delta ; \Gamma \vdash \diamond A \downarrow \quad \Delta ; A \vdash \diamond C \uparrow}{\left.\mathcal{D}_{1}\left(\mathcal{D}_{2}\right)\right)} \\
& \stackrel{\Delta ; \Gamma \vdash \diamond C \uparrow}{\text { i.h. }}=\frac{\Delta ; \Gamma \vdash \diamond A \downarrow \quad \Delta ; A \vdash \diamond C \uparrow}{\mathcal{D}_{1}} \Delta \mathrm{E} \\
& \frac{\Delta ; \Gamma \vdash \diamond C \uparrow}{\mathcal{D}_{2}}
\end{aligned}
$$

\section{Case:}

$$
\begin{aligned}
f\left(g\left(\frac{\Delta ; \Gamma \vdash A \downarrow}{\Delta ; \Gamma \vdash A \uparrow} \uparrow \downarrow\right)\right) & =f\left(g^{\prime}\left(\mathcal{D}_{1}, \overline{\Delta ; \Gamma \triangleright A \rightarrow A} \text { init }\right)\right) \\
& \stackrel{\text { i.h. }}{=} f^{\prime}\left(\overline{\Delta ; \Gamma \triangleright A \rightarrow A} \text { init, } \mathcal{D}_{1}\right) \\
& =\frac{\Delta ; \Gamma \vdash A \downarrow}{\Delta ; \Gamma \vdash A \uparrow} \uparrow
\end{aligned}
$$

Lemma 40 (Invertibility of $f$ and $g(2)$ ).

$$
\begin{aligned}
& \text { 1. If } \Delta ; \Gamma \underset{\mathcal{E}}{\rightarrow} \text {, then } g(f(\mathcal{D}))=\mathcal{D} \text {. } \\
& \text { 2. If } \Delta ; \Gamma \triangleright A \rightarrow C \text { and } \Delta ; \Gamma \vdash A \downarrow \text {, then } g\left(f^{\prime}(\mathcal{E}, \mathcal{F})\right)=g^{\prime}(\mathcal{F}, \mathcal{E}) \text {. }
\end{aligned}
$$

Proof. By simultaneous induction on the structure of the first given derivation. We show the base cases and the cases involving modalities.

\section{Case:}

$$
\begin{aligned}
g\left(f^{\prime}(\overline{\Delta ; \Gamma \triangleright A \rightarrow A} \text { init }, \mathcal{F})\right) & =g\left(\frac{\Delta ; \Gamma \vdash A \downarrow}{\Delta ; \Gamma \vdash A \uparrow} \uparrow\right) \\
& =g^{\prime}(\mathcal{F}, \overline{\Delta ; \Gamma \triangleright A \rightarrow A} \text { init })
\end{aligned}
$$

\section{Case:}

$$
\begin{aligned}
g\left(f^{\prime}(\overline{\Delta ; \Gamma \triangleright \perp \rightarrow A} \perp \mathrm{L}, \mathcal{F})\right) & =g\left(\frac{\Delta ; \Gamma \vdash \perp \downarrow}{\Delta ; \Gamma \vdash A \uparrow} \perp \mathrm{E}\right) \\
& =g^{\prime}(\mathcal{F}, \overline{\Delta ; \Gamma \triangleright \perp \rightarrow A} \perp \mathrm{L})
\end{aligned}
$$




\section{Case:}

$$
\begin{aligned}
& g\left(f\left(\frac{\Delta ; \Gamma_{1}, A, \Gamma_{2} \triangleright A \rightarrow C}{\Delta ; \Gamma_{1}, A, \Gamma_{2} \rightarrow C} \mathrm{ch}_{1}\right)\right)=g\left(f^{\prime}\left(\mathcal{D}_{1}, \overline{\Delta ; \Gamma_{1}, A, \Gamma_{2} \vdash A \downarrow} \text { hyp }_{1}\right)\right) \\
& \stackrel{\text { i.h. }}{=} g^{\prime}\left(\overline{\Delta ; \Gamma_{1}, A, \Gamma_{2} \vdash A \downarrow} \text { hyp }_{1}, \mathcal{D}_{1}\right) \\
& \mathcal{D}_{1} \\
& =\frac{\Delta ; \Gamma_{1}, A, \Gamma_{2} \triangleright A \rightarrow C}{\Delta ; \Gamma_{1}, A, \Gamma_{2} \rightarrow C} \mathrm{ch}_{1}
\end{aligned}
$$

\section{Case:}

$$
\begin{aligned}
& g\left(f\left(\frac{\Delta_{1}, A, \Delta_{2} ; \Gamma \triangleright A \rightarrow C}{\Delta_{1}, A, \Delta_{2} ; \Gamma \rightarrow C} \mathrm{ch}_{2}\right)\right)=g\left(f^{\prime}\left(\mathcal{D}_{1}, \frac{}{\Delta_{1}, A, \Delta_{2} ; \Gamma \vdash A \downarrow} \text { hyp }_{2}\right)\right) \\
& \stackrel{\text { i.h. }}{=} g^{\prime}\left(\overline{\Delta_{1}, A, \Delta_{2} ; \Gamma \vdash A \downarrow} \text { hyp }_{2}, \mathcal{D}_{1}\right) \\
& =\frac{\Delta_{1}, A, \Delta_{2} ; \Gamma \triangleright A \rightarrow C}{\Delta_{1}, A, \Delta_{2} ; \Gamma \rightarrow C} \mathrm{ch}_{2}
\end{aligned}
$$

Case:

$$
\begin{aligned}
& g\left(f\left(\begin{array}{c}
\mathcal{D}_{1} \\
\frac{\Delta ; \cdot \rightarrow A}{\Delta ; \Gamma \rightarrow \square A} \square \mathrm{R}
\end{array}\right)\right)=g\left(\begin{array}{c}
f\left(\mathcal{D}_{1}\right) \\
\frac{\Delta ; \cdot \vdash A \uparrow}{\Delta ; \Gamma \vdash \square A \uparrow} \square \mathrm{l}
\end{array}\right) \\
& g\left(f\left(\mathcal{D}_{1}\right)\right) \\
& =\frac{\Delta ; \cdot \rightarrow A}{\Delta ; \Gamma \rightarrow \square A} \square \mathrm{R} \\
& \stackrel{\text { i.h. }}{=} \frac{\Delta ; \cdot \rightarrow A}{\Delta ; \Gamma \rightarrow \square A} \square \mathrm{R}
\end{aligned}
$$

\section{Case:}

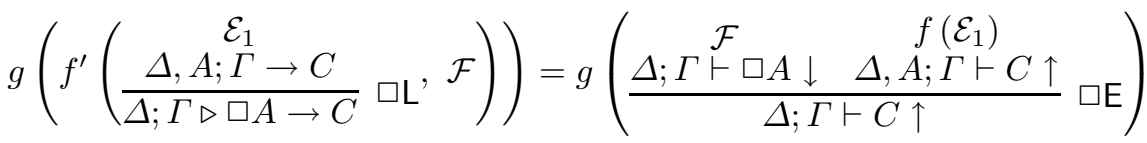

$$
\begin{aligned}
& =g^{\prime}\left(\begin{array}{c}
g\left(f\left(\mathcal{E}_{1}\right)\right) \\
\mathcal{F}, \frac{\Delta, A ; \Gamma \rightarrow C}{\Delta ; \Gamma \triangleright \square A \rightarrow C} \square \mathrm{L}
\end{array}\right) \\
& \stackrel{\text { i.h. }}{=} g^{\prime}\left(\mathcal{F}, \frac{\Delta, A ; \Gamma \rightarrow C}{\mathcal{E}_{1}}, \quad \square \mathrm{L}\right)
\end{aligned}
$$

\section{Case:}

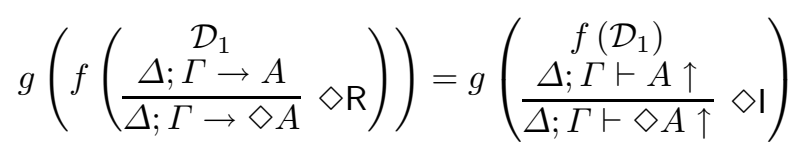




$$
\begin{aligned}
& =\frac{g\left(f\left(\mathcal{D}_{1}\right)\right)}{=}=\frac{\Delta ; \Gamma \rightarrow A}{\Delta ; \Gamma \rightarrow \diamond A} \diamond \mathrm{R} \\
& \stackrel{\mathcal{D}_{1}}{=} \frac{\Delta ; \Gamma \rightarrow A}{\Delta ; \Gamma \rightarrow \diamond A} \diamond \mathrm{R}
\end{aligned}
$$

\section{Case:}

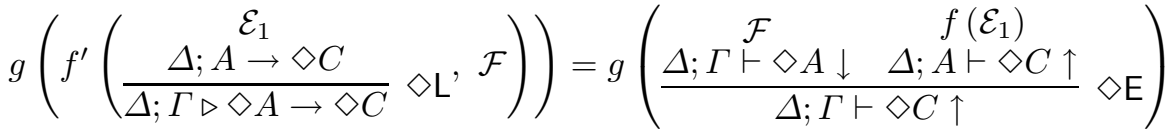

$$
\begin{aligned}
& =g^{\prime}\left(\mathcal{F}, \frac{g\left(f\left(\mathcal{E}_{1}\right)\right)}{\Delta ; A \rightarrow \diamond C} \underset{\Delta ; \Gamma \triangleright \diamond A \rightarrow \diamond C}{\Delta \mathrm{L}}\right) \\
& \stackrel{\text { i.h. }}{=} g^{\prime}\left(\mathcal{F}, \frac{\Delta ; A \rightarrow \diamond C}{\Delta ; \Gamma \triangleright \diamond A \rightarrow \diamond C} \diamond \mathrm{L}\right)
\end{aligned}
$$

A function is bijective if and only if it is both left- and right-invertible. As a result, the bijectivity of both $f$ and $g$ follows immediately from Lemmas 39 and 40. The soundness and completeness of $\mathbf{M} \mathbf{J}_{\mathbf{I S} 4}$ with respect to $\mathbf{N J}_{\mathbf{I S} 4}^{\mathbf{N}}$ follow directly from this result.

Theorem 11 (Bijection between $\mathrm{MJ}_{\mathrm{IS} 4}$ and $\mathrm{NJ}_{\mathrm{IS} 4}^{\mathrm{N}}$ derivations). Derivations of unfocused sequents in $\mathbf{M} \mathbf{J}_{\mathbf{I S} 4}$ correspond bijectively to derivations of $\uparrow$ judgements in $\mathbf{N} \mathbf{J}_{\mathbf{I S} 4}^{\mathbf{N}}$.

Proof. Functions $f$ and $g$, defined above, map derivations of unfocused sequents in $\mathbf{M} \mathbf{J}_{\mathbf{I S} 4}$ to derivations of $\uparrow$ judgements in $\mathbf{N} \mathbf{J}_{\mathbf{I S} 4}^{\mathbf{N}}$ and vice versa, respectively. The fact that $f$ and $g$ are bijections follows immediate from Lemmas 39 and 40.

Although $\mathbf{M J}_{\mathbf{I S} 4}$ is suitable for proof search in a backward direction, a naive approach still requires loop-detection to achieve a decision procedure. We will not pursue this direction further here, but instead concentrate on forward proof search, and on how we can combine ideas from backward and forward search to perform bidirectional proof search.

\section{Forward Proof Search}

Constructing $\mathbf{M} \mathbf{J}_{\text {IS4 }}$ proofs in a forward direction - from the top down - is complicated by the presence of multiple contexts, making $\mathbf{M} \mathbf{J}_{\mathbf{I S} 4}$ less than ideal for forward proof search. All $\mathbf{M} \mathbf{J}_{\mathbf{I S} 4}$ derivations begin, at the leaves, with focused sequents of the form $\Delta ; \Gamma \triangleright A \rightarrow A$, with $A$ atomic. After a sequence of (possibly zero) left-rule applications, the stoup formula is dropped from the stoup into one 
of the contexts by an application of $\mathrm{ch}_{1}$ or $\mathrm{ch}_{2}$. In a focused forward calculus used as the basis for the inverse method [5], we would proceed in a similar way, but it is not clear which context a stoup formula should be dropped into.

To address this uncertainty, we refine the idea of focusing and develop the system $\mathbf{M J} \mathbf{J}_{\mathbf{I S 4}}^{\mathbf{F}}$, which is suitable for forward proof search and features sequents of three kinds, involving both modal and nonmodal stoups:

$$
\begin{array}{ll}
\Delta ; \Gamma \mapsto C & C \text { can be proved using all assumptions in } \Delta, \Gamma, \\
\Delta ; \Gamma \triangleright A \mapsto C & C \text { can be proved using all assumptions in } \Delta, \Gamma, A \text {, with } \\
& A \text { assumed true, } \\
\Delta ; \Gamma \triangleright \triangleright A \mapsto C & C \text { can be proved using all assumptions in } \Delta, \Gamma, A, \text { with } \\
& A \text { assumed valid. }
\end{array}
$$

Note that the forms of the focused sequents reveal which context the stoup formula will drop into. For brevity, we write $\Delta ; \Gamma \triangleright^{i} A \mapsto C, i \in\{1,2\}$ for either form of focused sequent.

The inference rules of $\mathbf{M} \mathbf{J}_{\mathbf{I S} 4}^{\mathbf{F}}$, shown in Fig. 6, are obtained by reinterpreting the rules of $\mathbf{M} \mathbf{J}_{\mathbf{I S 4}}$ in a forward fashion and by defining the $\mathrm{ch}_{i}$ rules to behave as sketched above. The contexts of $\mathbf{M} \mathbf{J}_{\mathbf{I S 4}}^{\mathbf{F}}$, however, are interpreted differently, in that sequents $\Delta ; \Gamma \mapsto C$ and $\Delta ; \Gamma \triangleright^{i} A \mapsto C, i \in\{1,2\}$ assert that all assumptions in $\Delta$ and $\Gamma$, as well as $A$ if the sequent is focused, are needed to prove $C$. General weakening, which holds in $\mathbf{M} \mathbf{J}_{\mathbf{I S} 4}$, is thus disallowed, but local weakening is incorporated in the rule $\supset \mathrm{R}_{2}$. Contexts in $\mathbf{M} \mathbf{J}_{\mathbf{I S} 4}^{\mathbf{F}}$ are treated as sets rather than multisets, and we write $\Gamma_{1}, \Gamma_{2}$ and $\Gamma, A$ for $\Gamma_{1} \cup \Gamma_{2}$ and $\Gamma \cup\{A\}$, respectively. Note that a rule such as

$$
\frac{\Delta_{1} ; \Gamma_{1}, A_{1} \mapsto C \quad \Delta_{2} ; \Gamma_{2}, A_{2} \mapsto C}{\Delta_{1}, \Delta_{2} ; \Gamma_{1}, \Gamma_{2} \triangleright^{i} A_{1} \vee A_{2} \mapsto C} \vee L_{i}
$$

could more pedantically be written

$$
\frac{\Delta_{1} ; \Gamma_{1} \mapsto C \quad \Delta_{2} ; \Gamma_{2} \mapsto C}{\Delta_{1} \cup \Delta_{2} ;\left(\Gamma_{1} \backslash\left\{A_{1}\right\}\right) \cup\left(\Gamma_{2} \backslash\left\{A_{2}\right\}\right) \triangleright^{i} A_{1} \vee A_{2} \mapsto C} \vee L_{i} \quad\left(A_{1} \in \Gamma_{1}, A_{2} \in \Gamma_{2}\right)
$$

but we use the former notation for readability and consistency with our previous systems. No essential ambiguity is introduced by this convention.

In the following soundness and completeness results, we tacitly convert contexts from lists to sets and vice versa. More precisely, if $\Delta$ and $\Gamma$ are $\mathbf{M} \mathbf{J}_{\text {IS4 }}$ contexts, then when used in $\mathbf{M J} \mathbf{J}_{\mathbf{I S} 4}^{\mathbf{F}}$ sequents $\Delta ; \Gamma \mapsto C$ or $\Delta ; \Gamma \triangleright^{i} A \mapsto C$, they are converted into the sets that contain all distinct formulas in the contexts $\Delta$ and $\Gamma$. Similarly, if $\Delta$ and $\Gamma$ are sets of formulas, that is, $\mathbf{M} \mathbf{J}_{\mathbf{I S} 4}^{\mathbf{F}}$ contexts, then when used in $\mathbf{M} \mathbf{J}_{\mathbf{I S} 4}$ sequents $\Delta ; \Gamma \rightarrow C$ or $\Delta ; \Gamma \triangleright A \rightarrow C$, they are converted into lists containing all elements in the sets $\Delta$ and $\Gamma$. As a shorthand, if $\Gamma$ is a list and $\Gamma^{\prime}$ is a set, then we write $\Gamma^{\prime} \subseteq \Gamma$ to mean that every element of $\Gamma^{\prime}$ is contained in $\Gamma$.

\section{Theorem 12 (Soundness of $\mathrm{MJ} \mathrm{IS}_{\mathrm{I}}^{\mathrm{F}}$ with respect to $\mathrm{MJ} \mathrm{J}_{\mathrm{IS} 4}$ ).}




$$
\begin{aligned}
& \overline{; \cdot \triangleright^{i} A \mapsto A} \text { init }_{i} \quad(A \text { is atomic }) \quad \overline{; \cdot \triangleright^{i} \perp \mapsto A} \perp \mathrm{L}_{i} \\
& \frac{\Delta ; \Gamma \triangleright A \mapsto C}{\Delta ; \Gamma, A \mapsto C} \operatorname{ch}_{1} \frac{\Delta ; \Gamma \triangleright \triangleright A \mapsto C}{\Delta, A ; \Gamma \mapsto C} \operatorname{ch}_{2} \\
& \frac{\Delta ; \Gamma, A_{1} \mapsto A_{2}}{\Delta ; \Gamma \mapsto A_{1} \supset A_{2}} \supset \mathrm{R}_{1} \quad \frac{\Delta ; \Gamma \mapsto A_{2}}{\Delta ; \Gamma \mapsto A_{1} \supset A_{2}} \supset \mathrm{R}_{2} \\
& \frac{\Delta_{1} ; \Gamma_{1} \mapsto A_{1} \quad \Delta_{2} ; \Gamma_{2} \triangleright^{i} A_{2} \mapsto C}{\Delta_{1}, \Delta_{2} ; \Gamma_{1}, \Gamma_{2} \triangleright^{i} A_{1} \supset A_{2} \mapsto C} \supset \mathrm{L}_{i} \\
& \frac{\Delta_{1} ; \Gamma_{1} \mapsto A_{1} \quad \Delta_{2} ; \Gamma_{2} \mapsto A_{2}}{\Delta_{1}, \Delta_{2} ; \Gamma_{1}, \Gamma_{2} \mapsto A_{1} \wedge A_{2}} \wedge \mathrm{R} \frac{\Delta ; \Gamma \triangleright^{i} A_{j} \mapsto C}{\Delta ; \Gamma \triangleright^{i} A_{1} \wedge A_{2} \mapsto C} \wedge \mathrm{L}_{i, j} \\
& \frac{\Delta ; \Gamma \mapsto A_{j}}{\Delta ; \Gamma \mapsto A_{1} \vee A_{2}} \vee \mathrm{R}_{j} \frac{\Delta_{1} ; \Gamma_{1}, A_{1} \mapsto C \quad \Delta_{2} ; \Gamma_{2}, A_{2} \mapsto C}{\Delta_{1}, \Delta_{2} ; \Gamma_{1}, \Gamma_{2} \triangleright^{i} A_{1} \vee A_{2} \mapsto C} \vee \mathrm{L}_{i} \\
& \frac{\Delta ; \cdot \mapsto A}{\Delta ; \cdot \mapsto \square A} \square \mathrm{R} \quad \frac{\Delta, A ; \Gamma \mapsto C}{\Delta ; \Gamma \triangleright^{i} \square A \mapsto C} \square \mathrm{L}_{i} \quad \frac{\Delta ; \Gamma \mapsto A}{\Delta ; \Gamma \mapsto \diamond A} \diamond \mathrm{R} \quad \frac{\Delta ; A \mapsto \diamond C}{\Delta ; \cdot \triangleright^{i} \diamond A \mapsto \diamond C} \diamond \mathrm{L}_{i} \\
& i, j \in\{1,2\}
\end{aligned}
$$

Fig. 6. $\mathrm{MJ}_{\mathrm{IS} 4}^{\mathrm{F}}$
1. If $\Delta ; \stackrel{\mathcal{D}}{\mapsto} \mathcal{E} C$, then $\Delta ; \Gamma \rightarrow C$.
2. If $\Delta ; \Gamma \triangleright^{i} A \mapsto C, i \in\{1,2\}$, then $\Delta ; \Gamma \triangleright A \rightarrow C$.

Proof. By simultaneous induction on the structure of the given derivation. We show the base cases and the cases involving modalities.

\section{Case:}

$$
\mathcal{E}=\overline{; \cdot \triangleright^{i} A \mapsto A} \text { init }_{i}
$$

(1) $; \cdot \triangleright A \rightarrow A$

Case:

$$
\mathcal{E}=\overline{\cdot ; \cdot \triangleright^{i} \perp \mapsto A} \perp \mathrm{L}_{i}
$$

(1) $; \cdot \triangleright \perp \rightarrow A$

Case:

$$
\mathcal{D}=\frac{\mathcal{D}_{1}}{\Delta ; \Gamma \triangleright A \mapsto C} \quad \operatorname{ch}_{1} \quad(A \in \Gamma)
$$

(1) $\Delta ; \Gamma \triangleright A \rightarrow C$

i.h. $\left(\mathcal{D}_{1}\right)$

(2) $\Delta ; \Gamma \rightarrow C$

\section{Case:}

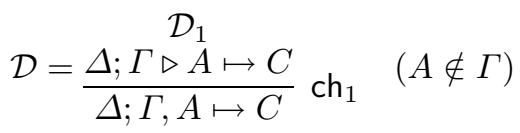


(1) $\Delta ; \Gamma \triangleright A \rightarrow C$

(2) $\Delta ; \Gamma, A \triangleright A \rightarrow C$

(3) $\Delta ; \Gamma, A \rightarrow C$ i.h. $\left(\mathcal{D}_{1}\right)$

Lemma 34 (1)

$\mathrm{ch}_{1}(2)$

Case:

$$
\mathcal{D}=\frac{\stackrel{\mathcal{D}_{1}}{\Delta ; \Gamma \triangleright \triangleright A \mapsto C}}{\Delta, A ; \Gamma \mapsto C} \operatorname{ch}_{2} \quad(A \in \Delta)
$$

(1) $\Delta ; \Gamma \triangleright A \rightarrow C$

i.h. $\left(\mathcal{D}_{1}\right)$

(2) $\Delta ; \Gamma \rightarrow C$ $\mathrm{ch}_{2}(1)$

Case:

$$
\mathcal{D}=\frac{\mathcal{D}_{1}}{\Delta ; \Gamma \triangleright \triangleright A \mapsto C} \quad \operatorname{ch}_{2} \quad(A \notin \Delta)
$$
(1) $\Delta ; \Gamma \triangleright A \rightarrow C$
i.h. $\left(\mathcal{D}_{1}\right)$
(2) $\Delta, A ; \Gamma \triangleright A \rightarrow C$
Lemma 35 (1)
(3) $\Delta, A ; \Gamma \rightarrow C$
$\mathrm{ch}_{2}(2)$

Case:

$$
\mathcal{D}=\frac{\Delta ; \cdot \stackrel{\mathcal{D}_{1}}{\Delta ;}}{\Delta ; \cdot \mapsto \square A} \square \mathrm{R}
$$

(1) $\Delta ; \cdot \rightarrow A$

(2) $\Delta ; \cdot \rightarrow \square A$

i.h. $\left(\mathcal{D}_{1}\right)$ $\square \mathrm{R}(1)$

\section{Case:}

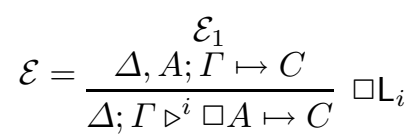

(1) $\Delta, A ; \Gamma \rightarrow C$

(2) $\Delta ; \Gamma \triangleright \square A \rightarrow C$

Case:

$$
\mathcal{D}=\frac{\Delta ; \Gamma \mapsto A}{\mathcal{D}_{1}} \diamond \mathrm{R}
$$

(1) $\Delta ; \Gamma \rightarrow A$

(2) $\Delta ; \Gamma \rightarrow \diamond A$

i.h. $\left(\mathcal{D}_{1}\right)$ $\diamond \mathrm{R}(1)$

\section{Case:}

$$
\mathcal{E}=\frac{\Delta ; A \stackrel{\mathcal{E}_{1}}{\Delta \mapsto C}}{\Delta ; \cdot \triangleright^{i} \diamond A \mapsto \diamond C} \diamond \mathrm{L}_{i}
$$

$$
\begin{aligned}
& \text { (1) } \Delta ; A \rightarrow \diamond C \\
& \text { (2) } \Delta ; \triangleright \diamond A \rightarrow \diamond C
\end{aligned}
$$

The statement of the completeness result is slightly nonstandard. This has two reasons, the first being that the focusing mechanism of $\mathbf{M} \mathbf{J}_{\mathbf{I S} 4}$ has the role of both types of focusing in $\mathbf{M} \mathbf{J}_{\mathbf{I S} 4}^{\mathbf{F}}$, so some of the behaviour of the $\mathbf{M} \mathbf{J}_{\mathbf{I S} 4}^{\mathbf{F}}$ 
focusing mechanism needs to be extracted from the corresponding $\mathbf{M} \mathbf{J}_{\mathbf{I S} 4}$ focused sequents. The second reason is that a derivation in $\mathbf{M J}_{\mathbf{I S} 4}$ can contain redundant focused segments, which add formulas to the contexts that are not strictly necessary. Since the construction of such focused segments is not possible in $\mathbf{M} \mathbf{J}_{\mathbf{I S} 4}^{\mathbf{F}}$ (since all formulas in the contexts of a sequent are required to prove its goal formula), we can ignore these redundant focusings completely in converting $\mathbf{M} \mathbf{J}_{\mathbf{I S} 4}$ derivations into $\mathbf{M} \mathbf{J}_{\mathbf{I S} 4}^{\mathbf{F}}$ derivations.

\section{Theorem 13 (Completeness of $\mathrm{MJ}_{\mathrm{IS} 4}^{\mathrm{F}}$ with respect to $\mathrm{MJ}_{\mathrm{IS} 4}$ ).}

1. If $\Delta ; \stackrel{\mathcal{D}}{\rightarrow} C$, then $\Delta^{\prime} ; \Gamma^{\prime} \mapsto C$ for some $\Delta^{\prime} \subseteq \Delta, \Gamma^{\prime} \subseteq \Gamma$.

2. If $\Delta ; \Gamma \triangleright A \rightarrow C$ and $A$ is a subformula of a formula in $\Gamma$, then either $\Delta^{\prime} ; \Gamma^{\prime} \mapsto \underset{\mathcal{F}}{C}$ or $\Delta^{\prime} ; \Gamma^{\prime} \triangleright A \mapsto C$ for some $\Delta^{\prime} \subseteq \Delta, \Gamma^{\prime} \subseteq \Gamma$.

3. If $\Delta ; \Gamma \triangleright A \rightarrow C$ and $A$ is a subformula of a formula in $\Delta$, then either $\Delta^{\prime} ; \Gamma^{\prime} \mapsto C$ or $\Delta^{\prime} ; \Gamma^{\prime} \triangleright \triangleright A \mapsto C$ for some $\Delta^{\prime} \subseteq \Delta, \Gamma^{\prime} \subseteq \Gamma$.

Proof. By simultaneous induction on the structure of the given derivation. We show the base cases and the cases involving modalities.

Case:

$$
\mathcal{E}=\overline{\Delta ; \Gamma \triangleright A \rightarrow A} \text { init }(A \text { is a subformula of a formula in } \Gamma)
$$

(1) $\cdot ; \cdot \triangleright A \mapsto A \quad$ init $_{1}$

\section{Case:}

$$
\mathcal{F}=\overline{\Delta ; \Gamma \triangleright A \rightarrow A} \text { init } \quad(A \text { is a subformula of a formula in } \Delta)
$$
(1) $\cdot ; \cdot \triangleright \triangleright A \mapsto A$
init $_{2}$

Case:

$$
\mathcal{E}=\overline{\Delta ; \Gamma \triangleright \perp \rightarrow A} \perp \mathrm{L} \quad(\perp \text { is a subformula of a formula in } \Gamma)
$$

(1) $\cdot ; \cdot \triangleright \perp \mapsto A$

Case:

$$
\mathcal{F}=\overline{\Delta ; \Gamma \triangleright \perp \rightarrow A} \perp \mathbf{L} \quad(\perp \text { is a subformula of a formula in } \Delta)
$$

(1) $\cdot ; \cdot \triangleright \triangleright \perp \mapsto A$

\section{Case:}

$$
\mathcal{D}=\frac{\mathcal{D}_{1}}{\Delta ; \Gamma_{1}, A, \Gamma_{2} \triangleright A \rightarrow C} \mathrm{ch}_{1}
$$

Subcase:

(1) $\Delta^{\prime} ; \Gamma^{\prime} \mapsto C$

i.h. $\left(\mathcal{D}_{1}\right)$

Subcase:

(1) $\Delta^{\prime} ; \Gamma^{\prime} \triangleright A \mapsto C$

i.h. $\left(\mathcal{D}_{1}\right)$

(2) $\Delta^{\prime} ; \Gamma^{\prime}, A \mapsto C$ 


\section{Case:}

$$
\mathcal{D}=\frac{\mathcal{D}_{1}}{\Delta_{1}, A, \Delta_{2} ; \Gamma \triangleright A \rightarrow C} \mathrm{ch}_{2}
$$

\section{Subcase:}
(1) $\Delta^{\prime} ; \Gamma^{\prime} \mapsto C$
i.h. $\left(\mathcal{D}_{1}\right)$

Subcase:
(1) $\Delta^{\prime} ; \Gamma^{\prime} \triangleright \triangleright A \mapsto C$
i.h. $\left(\mathcal{D}_{1}\right)$
(2) $\Delta^{\prime}, A ; \Gamma^{\prime} \mapsto C$

Case:

$$
\mathcal{D}=\frac{\Delta ; \cdot \stackrel{\mathcal{D}_{1}}{\rightarrow} A}{\Delta ; \Gamma \rightarrow \square A} \square \mathrm{R}
$$
(1) $\Delta^{\prime} ; \cdot \mapsto A$
i.h. $\left(\mathcal{D}_{1}\right)$
(2) $\Delta^{\prime} ; \cdot \mapsto \square A$
$\square \mathrm{R}(1)$

\section{Case:}

$$
\mathcal{E}=\frac{\mathcal{E}_{1}}{\Delta ; A ; \Gamma \rightarrow C} \quad \square \mathrm{L} \quad(\square A \text { is a subformula of a formula in } \Gamma)
$$

\section{Subcase:}

(1) $\Delta^{\prime} ; \Gamma^{\prime} \mapsto C \quad\left(A \notin \Delta^{\prime}\right) \quad$ i.h. $\left(\mathcal{E}_{1}\right)$ Subcase:

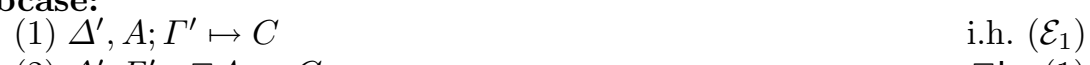

Case:

(2) $\Delta^{\prime} ; \Gamma^{\prime} \triangleright \square A \mapsto C$

$$
\mathcal{F}=\frac{\mathcal{F}_{1}}{\Delta, A ; \Gamma \rightarrow C} \quad \square \mathrm{L} \quad(\square A \text { is a subformula of a formula in } \Delta)
$$

\section{Subcase:}

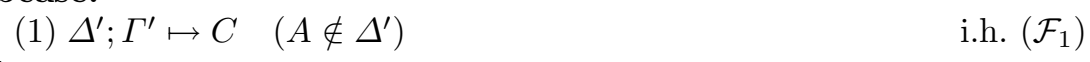

Subcase:

(1) $\Delta^{\prime}, A ; \Gamma^{\prime} \mapsto C$

(2) $\Delta^{\prime} ; \Gamma^{\prime} \triangleright \triangleright \square A \mapsto C$

Case:

$$
\mathcal{D}=\frac{\Delta ; \Gamma_{1}^{\mathcal{D}_{1}} \rightarrow A}{\Delta ; \Gamma \rightarrow \diamond A} \diamond \mathrm{R}
$$
(1) $\Delta^{\prime} ; \Gamma^{\prime} \mapsto A$
i.h. $\left(\mathcal{D}_{1}\right)$
(2) $\Delta^{\prime} ; \Gamma^{\prime} \mapsto \diamond A$

\section{Case:}

$$
\mathcal{E}=\frac{\Delta ; A \rightarrow \diamond C}{\Delta ; \Gamma \triangleright \diamond A \rightarrow \diamond C} \diamond \mathrm{L} \quad(\diamond A \text { is a subformula of a formula in } \Gamma)
$$




\section{Subcase:}

(1) $\Delta^{\prime} ; \cdot \mapsto \diamond C \quad$ i.h. $\left(\mathcal{E}_{1}\right)$

Subcase:

(1) $\Delta^{\prime} ; A \mapsto \diamond C \quad$ i.h. $\left(\mathcal{E}_{1}\right)$

Case:

(2) $\Delta^{\prime} ; \cdot \triangleright \diamond A \mapsto \diamond C \quad \diamond \mathrm{L}_{1}(1)$

$$
\mathcal{F}=\frac{\Delta ; A \rightarrow \diamond C}{\Delta ; \Gamma \triangleright \diamond A \rightarrow \diamond C} \diamond \mathrm{L} \quad(\diamond A \text { is a subformula of a formula in } \Delta)
$$

\section{Subcase:}

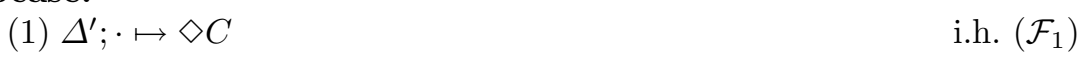

Subcase:

(1) $\Delta^{\prime} ; A \mapsto \diamond C \quad$ i.h. $\left(\mathcal{F}_{1}\right)$

(2) $\Delta^{\prime} ; \cdot \triangleright \triangleright \diamond A \mapsto \diamond C \quad \diamond \mathrm{L}_{2}(1)$

Note that the more fine-grained focusing mechanism of $\mathbf{M} \mathbf{J}_{\mathbf{I S} \mathbf{4}}^{\mathbf{F}}$ could just as well have been introduced in a sequent calculus suitable for backward reasoning, such as $\mathbf{M} \mathbf{J}_{\mathbf{I S} 4}$. Indeed, the single type of focused sequent in $\mathbf{M} \mathbf{J}_{\mathbf{I S} \mathbf{4}}$ has the role of both types of focused sequents in $\mathbf{M} \mathbf{J}_{\mathbf{I S} 4}^{\mathbf{F}}$, making the focusing mechanism of MJ $\mathbf{I S}_{\text {IS }}$ in some sense "overloaded".

The forward calculus $\mathbf{M} \mathbf{J}_{\mathbf{I S 4}}^{\mathbf{F}}$ suggests itself immediately as a basis for an implementation of the inverse method [5], fundamental to which is the classification of the subformulas of a query formula into positive and negative classes. The sign of a subformula determines where in a sequent it may occur (for instance as a goal formula or in the context) and restricts nondeterminism during proof search. We will refine this notion by classifying subformulas as either

1. positive $(+)$ subformulas, which may occur as goal formulas,

2. negative (-) subformulas, which may occur in the nonmodal context,

3. negative focused $(\sim)$ subformulas, which may occur in the nonmodal stoup,

4. valid (=) subformulas, which may occur in the modal context, or

5 . valid focused $(\approx)$ subformulas, which may occur in the modal stoup.

With this intended interpretation, it is straightforward to read the formal definition of refined signed subformulas directly from the inference rules of $\mathbf{M} \mathbf{J}_{\mathbf{I S} 4}^{\mathbf{F}}$.

Definition 1 (Signed subformulas).

$A$ signed subformula $A^{*}$ is a formula $A$ with a sign $* \in\{+,-, \sim,=, \approx\}$. The subformula relation $\leq$ is the smallest reflexive and transitive relation between signed subformulas satisfying the following.

$$
\begin{aligned}
A_{1}^{-}, A_{2}^{+} \leq & \left(A_{1} \supset A_{2}\right)^{+} \quad A_{i}^{+} \leq\left(A_{1} \wedge A_{2}\right)^{+} \quad A_{i}^{+} \leq\left(A_{1} \vee A_{2}\right)^{+} \\
& A^{+} \leq(\square A)^{+} \quad A^{+} \leq(\diamond A)^{+} \quad A^{\sim} \leq A^{-} \\
A_{1}^{+}, A_{2}^{\sim} \leq & \left(A_{1} \supset A_{2}\right)^{\sim} \quad A_{i}^{\sim} \leq\left(A_{1} \wedge A_{2}\right)^{\sim} \quad A_{i}^{-} \leq\left(A_{1} \vee A_{2}\right)^{\sim} \\
& A^{=} \leq(\square A)^{\sim} \quad A^{-} \leq(\diamond A)^{\sim} \quad A^{\approx} \leq A^{=}
\end{aligned}
$$




$$
\begin{aligned}
& \overline{\cdot ; \cdot \triangleright^{i} A^{*} \mapsto A^{+}} \text {init }_{i} \quad(A \text { is atomic }) \quad \overline{; \cdot \triangleright^{i} \perp^{*} \mapsto A^{+}} \perp \mathrm{L}_{i} \\
& \frac{\Delta^{=} ; \Gamma^{-} \triangleright A^{\sim} \mapsto C^{+}}{\Delta^{=} ; \Gamma^{-}, A^{-} \mapsto C^{+}} \operatorname{ch}_{1} \frac{\Delta^{=} ; \Gamma^{-} \triangleright \triangleright A^{\approx} \mapsto C^{+}}{\Delta^{=}, A^{=} ; \Gamma^{-} \mapsto C^{+}} \operatorname{ch}_{2} \\
& \frac{\Delta^{=} ; \Gamma^{-}, A_{1}^{-} \mapsto A_{2}^{+}}{\Delta^{=} ; \Gamma^{-} \mapsto\left(A_{1}^{-} \supset A_{2}^{+}\right)^{+}} \supset \mathrm{R}_{1} \quad \frac{\Delta^{=} ; \Gamma^{-} \mapsto A_{2}^{+}}{\Delta^{=} ; \Gamma^{-} \mapsto\left(A_{1}^{-} \supset A_{2}^{+}\right)^{+}} \supset \mathrm{R}_{2} \\
& \frac{\Delta_{1}^{=} ; \Gamma_{1}^{-} \mapsto A_{1}^{+} \quad \Delta_{2}^{\overline{=}} ; \Gamma_{2}^{-} \triangleright^{i} A_{2}^{*} \mapsto C^{+}}{\Delta_{1}^{\overline{=}}, \Delta_{2}^{\overline{=}} ; \Gamma_{1}^{-}, \Gamma_{2}^{-} \triangleright^{i}\left(A_{1}^{+} \supset A_{2}^{*}\right)^{*} \mapsto C^{+}} \supset \mathrm{L}_{i} \\
& \frac{\Delta_{1}^{=} ; \Gamma_{1}^{-} \mapsto A_{1}^{+} \quad \Delta_{2}^{=} ; \Gamma_{2}^{-} \mapsto A_{2}^{+}}{\Delta_{1}^{=}, \Delta_{2}^{=} ; \Gamma_{1}^{-}, \Gamma_{2}^{-} \mapsto\left(A_{1}^{+} \wedge A_{2}^{+}\right)^{+}} \wedge \mathrm{R} \frac{\Delta^{=} ; \Gamma^{-} \triangleright^{i} A_{j}^{*} \mapsto C^{+}}{\Delta^{=} ; \Gamma^{-} \triangleright^{i}\left(A_{1}^{*} \wedge A_{2}^{*}\right)^{*} \mapsto C^{+}} \wedge \mathrm{L}_{i, j}
\end{aligned}
$$

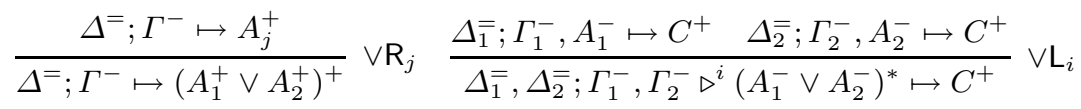

$$
\begin{aligned}
& \frac{\Delta^{=} ; \cdot \mapsto A^{+}}{\Delta^{=} ; \cdot \mapsto\left(\square A^{+}\right)^{+}} \square \mathrm{R} \quad \frac{\Delta^{=}, A^{=} ; \Gamma^{-} \mapsto C^{+}}{\Delta^{=} ; \Gamma^{-} \triangleright^{i}\left(\square A^{=}\right)^{*} \mapsto C^{+}} \square \mathrm{L}_{i} \\
& \frac{\Delta^{=} ; \Gamma^{-} \mapsto A^{+}}{\Delta^{=} ; \Gamma^{-} \mapsto\left(\diamond A^{+}\right)^{+}} \diamond \mathrm{R} \quad \frac{\Delta^{=} ; A^{-} \mapsto\left(\diamond C^{+}\right)^{+}}{\Delta^{=} ; \cdot \triangleright^{i}\left(\diamond A^{-}\right)^{*} \mapsto\left(\diamond C^{+}\right)^{+}} \diamond \mathrm{L}_{i} \\
& i, j \in\{1,2\}, * \text { is } \sim \text { or } \approx \text { if } i=1 \text { or } i=2 \text {, respectively. }
\end{aligned}
$$

Fig. 7. Signed MJ $\mathbf{J}_{\text {IS4 }}^{\mathbf{F}}$

$$
\begin{gathered}
A_{1}^{+}, A_{2} \leq\left(A_{1} \supset A_{2}\right) \approx A_{i} \leq\left(A_{1} \wedge A_{2}\right) \approx A_{i}^{-} \leq\left(A_{1} \vee A_{2}\right) \approx \\
A^{=} \leq(\square A) \approx A^{-} \leq(\diamond A) \approx \\
i \in\{1,2\}
\end{gathered}
$$

Note that for every negative subformula $A^{-}$of a signed formula $C^{*}, C^{*}$ also has, as a subformula, the corresponding negative focused subformula $A^{\sim}$. The converse, however, is not true in general. A signed formula $C^{*}$ may have a subformula $A^{\sim}$ without having a subformula $A^{-}$. A similar relation holds for valid and valid focused subformulas. The schematic formulas in the inference rules of $\mathbf{M} \mathbf{J}_{\mathbf{I S} 4}^{\mathbf{F}}$ can also be annotated with signs in a way that is consistent with the intended interpretation. Such a signed version of $\mathbf{M} \mathbf{J}_{\mathbf{I S} 4}^{\mathbf{F}}$ is shown in Fig. 7, where we write $\Gamma^{-}$and $\Delta^{=}$for contexts of signed subformulas of the forms $A_{1}^{-}, \ldots, A_{n}^{-}$and $B_{1}^{=}, \ldots, B_{m}^{=}$, respectively. From these signed inference rules, it is clear that the usual signed subformula property extends to encompass our refined signing scheme.

Theorem 14 (Signed subformula property).

Every sequent in a signed $\mathbf{M} \mathbf{J}_{\mathbf{I S} 4}^{\mathbf{F}}$ derivation

$$
\Delta^{=} ; \Gamma^{-} \mapsto C^{+} \quad \text { or } \quad \Delta^{=} ; \Gamma^{-} \triangleright^{i} A^{*} \mapsto C^{+}, i \in\{1,2\}
$$


where $*$ is $\sim$ or $\approx$ if $i=1$ or $i=2$, respectively, is of the form

1. $D_{1}^{=}, \ldots, D_{n}^{=} ; E_{1}^{-}, \ldots, E_{m}^{-} \mapsto F^{+}$,

2. $D_{1}^{=}, \ldots, D_{n}^{=} ; E_{1}^{-}, \ldots, E_{m}^{-} \triangleright E^{\sim} \mapsto F^{+}$, or

3. $D_{1}^{=}, \ldots, D_{n}^{=} ; E_{1}^{-}, \ldots, E_{m}^{-} \triangleright \triangleright D^{\approx} \mapsto F^{+}$,

where all $D_{j}^{\bar{j}}, E_{k}^{-}$, and $E^{\sim}, D^{\approx}$, and $F^{+}$are signed subformulas of $\Delta^{=}, \Gamma^{-}$, $C^{+}$, and $A^{*}$.

Proof. By simultaneous induction on the structure of the given derivation.

Theorem 14 guarantees, for instance, that in any signed $\mathbf{M} \mathbf{J}_{\mathbf{I S} 4}^{\mathbf{F}}$ derivation of $\Delta^{=} ; \Gamma^{-} \mapsto C^{+}$, all leaves are of the forms

$$
\overline{\cdot ; \cdot \triangleright^{i} A^{*} \mapsto A^{+}} \text {init }_{i} \quad \text { or } \quad \overline{; \cdot \triangleright^{i} \perp^{*} \mapsto B^{+}} \perp \mathrm{L}_{i} \quad i \in\{1,2\}
$$

where $A$ is atomic, $*$ is $\sim$ or $\approx$ if $i=1$ or $i=2$, respectively, and $A^{*}, A^{+}$, $\perp^{*}$, and $B^{+}$must be signed subformulas of $\Delta^{=}, \Gamma^{-}$, and $C^{+}$. In general, every rule application considered by an implementation of the inverse method must abide by the conditions set forth by the extended signed subformula property. This provides a foundation for a focused inverse method prover for IS4 with nondeterminism restricted more strongly than by the usual subformula property.

However, pure forward proof search techniques such as the inverse method also have shortcomings. For instance, the existence of two $\supset \mathrm{R}$ rules is a concession to the need for localized weakening, something usually handled more elegantly in backward decision procedures by general weakening. Also, the refined focusing we have introduced strongly restricts what rules are applicable, something that a decision procedure should be able to exploit in order to generate fewer intermediate sequents. These issues are addressed in the next section by combining ideas from forward and backward proof search.

\section{Bidirectional Proof Search in Sequent Calculus}

The idea behind the bidirectional sequent calculus method is that given a query formula $A$, we can, by exploiting forward proof search techniques, but before performing proof search itself, construct a set of derived inference rules for $\mathbf{M} \mathbf{J}_{\mathbf{I S} 4}$ which conceal all left-rule applications that could be needed in a proof of $A$. We then carry out backward proof search over these relevant derived rules and the usual right-rules of $\mathbf{M} \mathbf{J}_{\mathbf{I S 4}}$. By design, our derived inference rules will correspond exactly to the notion of focused threads in $\mathbf{M} \mathbf{J}_{\mathbf{I S} 4}^{\mathbf{F}}$ derivations, defined as follows.

\section{Definition 2 (Focused threads).}

$A$ focused thread of an $\mathbf{M} \mathbf{J}_{\mathbf{I S} 4}^{\mathbf{F}}$ derivation is a segment of the derivation that begins, at the top, with an application of init $t_{i}, \perp L_{i}, \vee L_{i}, \square L_{i}$, or $\diamond L_{i}, i \in\{1,2\}$ (raising a formula into a stoup), includes only focused sequents, and ends with an application of $\mathrm{ch}_{i}$ (dropping a formula from the stoup). 
In any $\mathbf{M} \mathbf{J}_{\mathbf{I S} 4}^{\mathbf{F}}$ derivation of an unfocused sequent, left-rule applications must occur in focused threads, so we can think of derivations as consisting of focused threads strung together using right-rule applications. The key insight is that all focused threads possibly needed in an $\mathbf{M} \mathbf{J}_{\mathbf{I S} 4}^{\mathbf{F}}$ proof of a formula $A$ can be deterministically constructed prior to proof search by inspecting the structure of $A$. To justify this claim, we will use our refined subformula property.

First note that it is straightforward to uniquely label subformula occurrences of a formula to be proved, and that the definition of signed subformulas, the signed subformula property, and the inference rules of $\mathbf{M} \mathbf{J}_{\mathbf{I S} \mathbf{F}}^{\mathbf{F}}$ can be adjusted to operate on labels rather than formulas, thus differentiating between subformula occurrences.

To give some intuition as to how to construct all the focused threads possibly needed for a proof of a formula, we will illustrate the approach on the following small example:

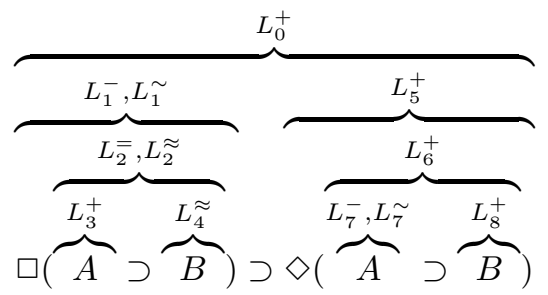

with signed subformula occurrences

$$
L_{0}^{+}, L_{3}^{+}, L_{5}^{+}, L_{6}^{+}, L_{8}^{+}, \quad L_{1}^{-}, L_{7}^{-}, \quad L_{1}^{\sim}, L_{7}^{\sim}, \quad L_{2}^{=}, \quad \text { and } \quad L_{2}^{\approx}, L_{4}^{\approx} .
$$

The signed subformula property guarantees that in a proof of the sequent $\cdot ; \cdot \mapsto$ $L_{0}^{+}$, the only axioms we require are

$$
\overline{; \cdot \triangleright L_{7}^{\widetilde{1}} \mapsto L_{3}^{+}} \text {init }_{1} \quad \text { and } \quad \overline{; \cdot \triangleright \triangleright L_{4}^{\approx} \mapsto L_{8}^{+}} \text {init }_{2}
$$

Consider the first of these axioms. Every applicable left-rule either drops the stoup formula occurrence into a context or expands it. The immediate parent of $L_{7}$ in the subformula tree is $L_{7}^{-}$, indicating that dropping the stoup formula occurrence into the context is a permissible operation. In fact, it is the only operation permitted by the signed subformula property operating on labels. We can collapse this short focused thread into a single derived inference rule:

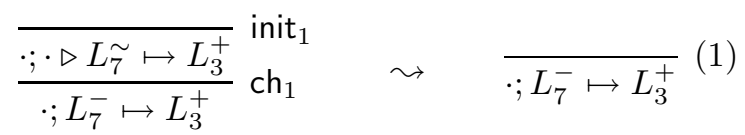

Considering the second axiom, we notice that the parent subformula occurrence of $L_{4} \approx$ is $L \approx \widetilde{2}$, also a focused subformula occurrence. The next rule application should then be $\supset \mathrm{L}_{2}$, with $L_{2} \approx$ as the principal formula occurrence. In fact, it is not difficult to see that since every subformula occurrence has a unique parent subformula occurrence, the signed subformula property operating on labels al-

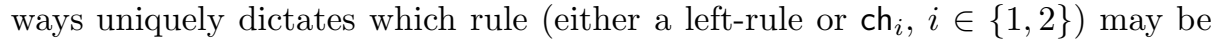


applied. This game continues until the end of the focused thread. In the case of the second axiom, the immediate parent of $L_{2} \widetilde{2}$ is $L_{2}^{\overline{=}}$, signalling an application of $\mathrm{ch}_{2}$ and the end of the thread:

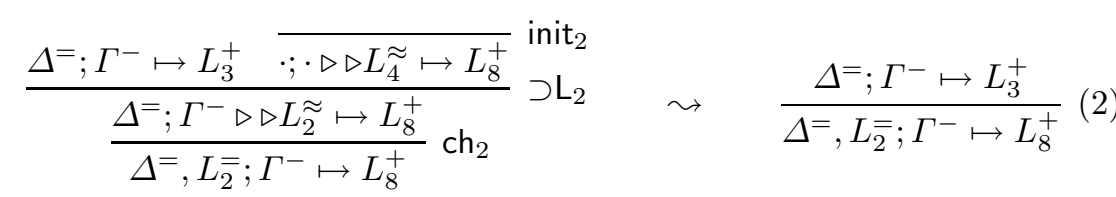

Note that this derived rule, unlike (1), has a premise and is parametric in the contexts $\Delta$ and $\Gamma$. Finally, the signed subformula property allows one more focused thread, starting with

$$
\frac{\Delta^{=}, L_{2}^{=} ; \Gamma^{-} \mapsto M^{+}}{\Delta^{=} ; \Gamma^{-} \triangleright L_{1}^{\sim} \mapsto M^{+}} \square \mathrm{L}_{1}
$$

The immediate parent subformula occurrence of $L_{1}^{\sim}$ is $L_{1}^{-}$, so this thread ends here, yielding the derived rule

$$
\frac{\frac{\Delta^{=}, L_{2}^{=} ; \Gamma^{-} \mapsto M^{+}}{\Delta^{=} ; \Gamma^{-} \triangleright L_{1}^{\sim} \mapsto M^{+}}}{\Delta^{=} ; \Gamma^{-}, L_{1}^{-} \mapsto M^{+}} \operatorname{ch}_{1} \quad \leadsto \quad \frac{\Delta^{=}, L_{2}^{=} ; \Gamma^{-} \mapsto M^{+}}{\Delta^{=} ; \Gamma^{-}, L_{1}^{-} \mapsto M^{+}}
$$

Notice that this derived rule is schematic not only in the contexts $\Delta$ and $\Gamma$, but also in the goal formula occurrence $M$. Such schematic representations of threads are not technically focused threads, according to our definition, but their concrete instantiations, when appearing in a derivation with concrete contexts and a goal formula, are the true focused threads corresponding to these schematic threads. To emphasize this distinction, we will occasionally refer to concrete focused threads or derived rules as focused thread instances or derived rule instances, respectively. Since the signed subformula property allows no other focused threads, the remainder of the proof, if one exists, may only chain the derived rules (1), (2), and (3) together with right-rule applications. In this case, completing the proof is straightforward:

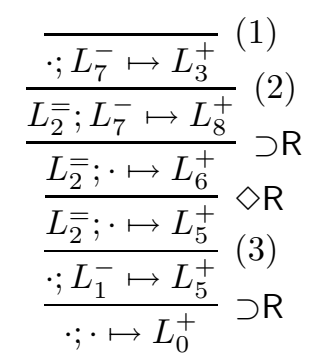

In general, to cover all focused threads that might be needed in a proof of the query formula, the construction of (possibly schematic) derived rules must begin with focused sequents of the following kinds, where $*$ is $\sim$ or $\approx$, depending on whether $i=1$ or $i=2$ : 
1. $\cdot ; \cdot \triangleright^{i} L_{j}^{*} \mapsto L_{k}^{+}$, where $L_{j}$ and $L_{k}$ denote the same atomic formula,

2. $\cdot ; \cdot \triangleright^{i} L_{j}^{*} \mapsto M^{+}$, where $L_{j}$ denotes $\perp$ and $M$ is schematic,

3. $\Delta^{=} ; \Gamma^{-} \triangleright^{i} L_{j}^{*} \mapsto M^{+}$, where $L_{j}$ denotes some $A_{1} \vee A_{2}$ and $M$ is schematic,

4. $\Delta^{=} ; \Gamma^{-} \triangleright^{i} L_{j}^{*} \mapsto M^{+}$, where $L_{j}$ denotes some $\square A$ and $M$ is schematic, and

5. $\Delta^{=} ; \Gamma^{-} \triangleright^{i} L_{j}^{*} \mapsto M^{+}$, where $L_{j}$ denotes some $\diamond A$ and $M$ is schematic, but denotes some $\diamond C$.

Moreover, the constructed derived rules must end with a stoup formula occurrence being dropped into one of the contexts.

The question now is how these forward-constructed derived rules can complement backward proof search. The key observation is that every focused thread of an $\mathbf{M} \mathbf{J}_{\mathbf{I S} 4}^{\mathbf{F}}$ derivation (schematic or concrete) can be converted into a focused thread of an $\mathbf{M J}_{\mathbf{I S} 4}$ derivation by applying weakening, reducing valid focused sequents to focused sequents, and omitting the now unnecessary signs of subformula labels. As one would expect, $\mathbf{M} \mathbf{J}_{\mathbf{I S} 4}$ focused threads are defined exactly like their $\mathbf{M} \mathbf{J}_{\mathbf{I S} 4}^{\mathbf{F}}$ counterparts: they begin with applications of init, $\perp \mathrm{L}, \mathrm{VL}, \square \mathrm{L}$, or $\diamond \mathrm{L}$, include only focused sequents, and end with applications of $\mathrm{ch}_{i}, i \in\{1,2\}$. For instance,

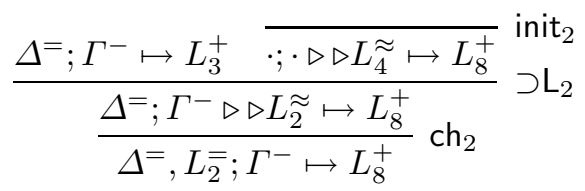

can be converted into the $\mathbf{M} \mathbf{J}_{\mathbf{I S} \mathbf{4}}$ focused thread

$$
\frac{\Delta_{1}, L_{2}, \Delta_{2} ; \Gamma \rightarrow L_{3} \quad \overline{\Delta_{1}, L_{2}, \Delta_{2} ; \Gamma \triangleright L_{4} \rightarrow L_{8}}}{\frac{\Delta_{1}, L_{2}, \Delta_{2} ; \Gamma \triangleright L_{2} \rightarrow L_{8}}{\Delta_{1}, L_{2}, \Delta_{2} ; \Gamma \rightarrow L_{8}} \mathrm{ch}_{2}} \supset \mathrm{L}
$$

where we have weakened some sequents, used $\mathbf{M J}_{\mathbf{I S} 4}$ 's single focusing mechanism to perform the duties of both focusing mechanisms of $\mathbf{M} \mathbf{J}_{\mathbf{I S} 4}^{\mathbf{F}}$, and dropped the subformula signs. We will, however, continue to treat formulas as labels, in order to distinguish between subformula occurrences.

This makes it possible to construct derived rules for $\mathbf{M} \mathbf{J}_{\mathbf{I S} 4}$, which will, in generally, be schematic. Note, however, that backward proof search over $\mathbf{M} \mathbf{J}_{\mathbf{I S} 4}$ with schematic derived rules is still not guaranteed to terminate, since rightrules and derived rules may be applied many times along a branch of a proof. There are several ways to overcome this problem. One appealing, if slightly naive approach, is to restrict the number of applications of schematic derived rules along a branch. In many cases, very short proofs are found by limiting the number of applications of derived rules along a branch to at most one. This also gives an immediate termination guarantee, since every rule application either reduces the number of available derived rules, or leaves the number of available derived rules unchanged, but reduces the complexity of the goal formula. This approach also does away with conventional loop-detection entirely. All that is needed is a way of keeping track of which schematic derived rules have been 
applied along a branch. While this bookkeeping apparatus is reminiscent of a history mechanism, we expect it to be far more lightweight than maintaining histories of previously encountered sequents or goal formulas, as is common in standard loop-detection schemes. Our expectations will, for the most part, be vindicated by our experimental results.

However, certain provable formulas, such as $(A \vee(A \supset B \vee C) \supset B \vee C) \supset$ $B \vee C$, do not have derivations where every schematic derived rule occurs at most once along a branch. In such a case, we can relax our restriction slightly, counting as derived rules not schematic focused threads, but particular focused thread instances, characterized by the identity of the schematic derived rule and the concrete goal formula occurrence, if applicable. The possible goal formulas potentially needed in a proof are simply the positive subformula occurrences of the query formula. While this may generate a large number of almost identical derived rule instances, the availability of these rules remains very simple to express: a rule instance is either available on a branch or not, and the bookeeping apparatus for available rule instances could be efficiently implemented as a bit vector, for instance.

It is possible to develop more fine-grained estimates of how many times a derived rule might be needed, and we have followed this approach in our implementation (see Sect. 9). Here, we will instead turn $\mathbf{M} \mathbf{J}_{\text {IS4 }}$ with forwardconstructed derived rules into a decision procedure using techniques familiar from loop-detection schemes using histories. As hinted at near the end of Sect. 5, it is possible to equip $\mathbf{M J}_{\mathbf{I S} 4}$ with a history mechanism much like Howe's [12]. A different approach which we will investigate here, related to the discussion above, is to precompute possible derived rule instances - schematic derived rules with all possible concrete goal formula occurrences - and, during proof search, to maintain lists or sets of which rule instances are still available. Unlike in the simple approach sketched above, however, the restrictions on how and how many times each rule instance can be applied along a branch are slightly more involved, in order to guarantee completeness.

To gain some more intuition regarding derived rule instances, recall that every $\mathbf{M J}_{\text {IS4 }}$ focused thread begins with an application of init, $\perp \mathrm{L}, \mathrm{VL}, \square \mathrm{L}$, or $\diamond \mathrm{L}$, includes only focused sequents, and ends with an application of $\mathrm{ch}_{i}, i \in\{1,2\}$. Fixing the goal formula occurrence $C$ and the stoup formula occurrence $A$ at the topmost rule application of the thread ${ }^{2}$ uniquely determines the shape of the focused thread, as justified by the signed subformula property in $\mathbf{M} \mathbf{J}_{\mathbf{I S} 4}^{\mathbf{F}}$ and the fact that the derived rule instances in $\mathbf{M} \mathbf{J}_{\mathbf{I S} \mathbf{4}}$ are constructed as in $\mathbf{M} \mathbf{J}_{\mathbf{I S 4} \mathbf{F}}^{\mathbf{F}}$. The goal formula occurrence of a focused thread instance does not change throughout the thread, since left-rule applications are independent of the goal formula. If the focused thread begins with an application of a rule that has premises ( $V \mathrm{~L}$, $\square \mathrm{L}$, or $\diamond \mathrm{L}$ ), we will call these premises, which still have $C$ as a goal formula occurrence, but whose contexts may differ from contexts in the focused thread, trunk premises or trunks. On the other hand, a focused thread can have open

\footnotetext{
${ }^{2}$ Although we will continue to think of formula occurrences as labels, we will use the metavariables $A, B, C$, etc. for readability.
} 
premises induced by applications of $\supset$ L. These premises, which we will call twig premises or twigs, have the same contexts as sequents in the focused thread, but different goal formula occurrences. If a focused thread begins with an application of a rule without premises (init or $\perp \mathrm{L}$ ), then it has no trunks.

Note that a focused thread instance need not have any twigs or trunks. The $\mathbf{M J}_{\mathbf{I S} 4}$ focused thread shown above (which corresponds to the $\mathbf{M} \mathbf{J}_{\mathbf{I S} 4}^{\mathbf{F}}$ derived rule (2) in the example shown previously) has one twig, $\Delta_{1}, L_{2}, \Delta_{2} ; \Gamma \rightarrow L_{3}$, and no trunks. The $\mathbf{M} \mathbf{J}_{\mathbf{I S} 4}$ focused thread corresponding to the $\mathbf{M} \mathbf{J}_{\mathbf{I S} 4}^{\mathbf{F}}$ derived rule (1) in the example has no twigs and no trunks, while every $\mathbf{M} \mathbf{J}_{\mathbf{I S} 4}$ focused thread instance corresponding to schematic derived rule (3) has one trunk and no twigs.

The general form of a $V L$ derived rule instance - that is, a derived rule instance concealing a focused thread starting with an application of $V L$ - for example, is

$$
\frac{\overbrace{\Delta ; \Gamma \rightarrow B_{1} \quad \cdots \quad \Delta ; \Gamma \rightarrow B_{n}}^{\text {twigs }} \overbrace{\Delta ; \Gamma, A_{1} \rightarrow C \quad \Delta ; \Gamma, A_{2} \rightarrow C}^{\text {trunks }}}{\Delta ; \Gamma \rightarrow C}
$$

while that of a $\diamond \mathrm{L}$ rule instance is

$$
\frac{\overbrace{\Delta ; \Gamma \rightarrow B_{1} \quad \cdots \quad \Delta ; \Gamma \rightarrow B_{n}}^{\text {twigs }} \overbrace{\Delta ; A_{1} \rightarrow \diamond C}^{\text {trunk }}}{\Delta ; \Gamma \rightarrow \diamond C}
$$

Building on $\mathbf{M} \mathbf{J}_{\mathbf{I S} 4}$, we will describe the system $\mathbf{M} \mathbf{J}_{\mathbf{I S} 4}^{\mathrm{B}}$, whose inference rules consist of derived rule instances - generated prior to proof search from a query formula as shown previously — and the usual right rules of $\mathbf{M} \mathbf{J}_{\mathbf{I S 4} 4}$. However, to guarantee completeness and termination of proof search in $\mathbf{M} \mathbf{J}_{\mathbf{I S} 4}^{\mathrm{B}}$, we require some additional provisos on the rules, inspired by loop-detection schemes using history mechanisms. Moreover, the treatment of some right rules of $\mathbf{M} \mathbf{J}_{\mathbf{I S} 4}^{\mathrm{B}}$ is not quite identical to that of $\mathbf{M} \mathbf{J}_{\mathbf{I S} 4}$. As a preliminary overview, the general approach of proof search in $\mathbf{M} \mathbf{J}_{\mathbf{I S} 4}^{\mathrm{B}}$ is to

1. precompute all derived rule instances (all focused threads, with schematic goal formulas instantiated with all suitable goal formula occurrences),

2. precompute all required instances of $\square \mathrm{R}$ applications (applications of $\square \mathrm{R}$, with schematic goal formulas instantiated with all suitable goal formula occurrences),

3. perform backward proof search over these rule instances and schematic inference rules for $\supset R, \wedge R, \vee R$, and $\diamond R$.

The intuition behind this approach is that we can statically control the availability of rule instances with conrete goal formulas while performing backward proof search, while the availabilities of the rules that we maintain in a schematic form $(\supset R, \wedge R, \vee R$, and $\diamond R)$ need not be controlled.

A sequent in $\mathbf{M} \mathbf{J}_{\mathbf{I S} 4}^{\mathrm{B}}$ consists not only of the two contexts and a goal formula, but also comprises a collection of available rule instances, as well as a collection of 
previously applied rule instances that may become available again if the contexts are modified along the current branch. More formally, an $\mathbf{M} \mathbf{J}_{\mathbf{I S} 4}^{\mathrm{B}}$ sequent has the form

$$
\Delta ; \Gamma \rightarrow C \| \square \mathrm{L}^{\mathcal{R}} ; \diamond \mathrm{L}^{\mathcal{R}} ; \vee \mathrm{L}^{\mathcal{R}} ; \perp \mathrm{L}^{\mathcal{R}} ; \text { init }^{\mathcal{R}} ; \square \mathrm{R}^{\mathcal{R}} ; \square \mathrm{L}^{\mathcal{T}} ; \diamond \mathrm{L}^{\mathcal{T}}
$$

where each $*^{\mathcal{R}}$ and $*^{\mathcal{T}}$ is a set of rule instances characterized by an application of $*$. We will assume that in the rule instance precomputation phase, unambiguous names or labels have been generated for rule instances, and that the complete sets of rules of each type (e.g. all derived rules starting with $\vee \mathrm{L}$ or $\square \mathrm{L}$ applications, etc.) are readily available. The $*^{\mathcal{R}}$ sets contain rule instances that are available for application along the current branch, while the $*^{\mathcal{T}}$ sets contain rule instance labels indicating which rule instances' twigs this sequent is located on. Although a rule instance will generally not immediately be available along its twigs, modifications to the contexts along the derivations rooted at the twigs can cause these rule instances to become available again. We will refer to the $*^{\mathcal{R}}$ and $*^{\mathcal{T}}$ sets associated with a sequent collectively as the sequent's bookkeeping sets. To avoid further cumbersome notation, we will provide an operational description of how the rule instances and schemas of $\mathbf{M} \mathbf{J}_{\mathbf{I S} 4}^{\mathrm{B}}$ are applied.

1. For a $\square \mathrm{L}$ derived rule instance

$$
\frac{\overbrace{\Delta ; \Gamma \rightarrow B_{1} \quad \cdots \quad \Delta ; \Gamma \rightarrow B_{n}}^{\text {twigs }} \overbrace{\Delta, A ; \Gamma \rightarrow C}^{\text {trunk }}}{\Delta ; \Gamma \rightarrow C} i
$$

with label $i$ to be applicable, the $\square \mathrm{L}^{\mathcal{R}}$ set of the conclusion must contain $i$. The bookkeeping rules of an application of rule instance $i$ are as follows.

(a) Copy the bookkeeping sets from the conclusion to each of the premises.

(b) Refill the $\diamond \mathrm{L}^{\mathcal{R}}, \square \mathrm{R}^{\mathcal{R}}, \mathrm{VL}^{\mathcal{R}}, \perp \mathrm{L}^{\mathcal{R}}$, and init ${ }^{\mathcal{R}}$ sets of the trunk - that is, let them be the full sets of precomputed rule instances of the appropriate types.

(c) Move the contents of the $\square \mathrm{L}^{\mathcal{T}}$ set of the trunk into the $\square \mathrm{L}^{\mathcal{R}}$ set of the trunk - that is, let the $\square \mathrm{L}^{\mathcal{R}}$ set of the trunk be $\square \mathrm{L}^{\mathcal{R}} \cup \square \mathrm{L}^{\mathcal{T}}$ and empty the $\square \mathrm{L}^{\mathcal{T}}$ set of the trunk. Empty the $\diamond \mathrm{L}^{\mathcal{T}}$ set of the trunk.

(d) Add $i$ to the $\square \mathrm{L}^{\mathcal{T}}$ set of each of the twigs.

(e) Remove $i$ from the $\square \mathrm{L}^{\mathcal{R}}$ set of each of the premises (both twigs and trunks).

2. For a $\diamond \mathrm{L}$ derived rule instance

$$
\frac{\overbrace{\Delta ; \Gamma \rightarrow B_{1} \quad \cdots \quad \Delta ; \Gamma \rightarrow B_{n}}^{\text {twigs }} \overbrace{\Delta ; A \rightarrow \diamond C}^{\text {trunk }}}{\Delta ; \Gamma \rightarrow \diamond C} i
$$

with label $i$ to be applicable, the $\diamond \mathrm{L}^{\mathcal{R}}$ set of the conclusion must contain $i$. The bookkeeping rules of an application of rule instance $i$ are as follows.

(a) Copy the bookkeeping sets from the conclusion to each of the premises.

(b) Refill the $\vee L^{\mathcal{R}}, \perp \mathrm{L}^{\mathcal{R}}$, and init ${ }^{\mathcal{R}}$ sets of the trunk. 
(c) Move the contents of the $\square \mathrm{L}^{\mathcal{T}}$ and $\diamond \mathrm{L}^{\mathcal{T}}$ sets of the trunk into, respectively, the $\square \mathrm{L}^{\mathcal{R}}$ and $\diamond \mathrm{L}^{\mathcal{R}}$ sets of the trunk.

(d) Add $i$ to the $\diamond \mathrm{L}^{\mathcal{T}}$ set of each of the twigs.

(e) Remove $i$ from the $\diamond \mathrm{L}^{\mathcal{R}}$ set of each of the premises.

3. For a $\vee \mathrm{L}$ derived rule instance

$$
\frac{\overbrace{\Delta ; \Gamma \rightarrow B_{1} \quad \cdots \quad \Delta ; \Gamma \rightarrow B_{n}}^{\text {twigs }} \overbrace{\Delta ; \Gamma, A_{1} \rightarrow C} \Delta ; \Gamma, A_{2} \rightarrow C}{\Delta ; \Gamma \rightarrow C} i
$$

with label $i$ to be applicable, the $\mathrm{VL}^{\mathcal{R}}$ set of the conclusion must contain $i$, and the context extensions in the trunks must be proper, that is, $A_{1}, A_{2} \notin \Gamma$. The bookkeeping rules of an application of rule instance $i$ are as follows.

(a) Copy the bookkeeping sets from the conclusion to each of the premises.

(b) Refill the $\vee L^{\mathcal{R}}, \perp \mathrm{L}^{\mathcal{R}}$, and init ${ }^{\mathcal{R}}$ sets of the trunks.

(c) Move the contents of the $\square \mathrm{L}^{\mathcal{T}}$ and $\diamond \mathrm{L}^{\mathcal{T}}$ sets of the trunks into, respectively, the $\square \mathrm{L}^{\mathcal{R}}$ and $\diamond \mathrm{L}^{\mathcal{R}}$ sets of the trunks.

(d) Remove $i$ from the $\mathrm{VL}^{\mathcal{R}}$ set of each of the premises.

4. For an init derived rule instance

$$
\overbrace{\Delta ; \Gamma \rightarrow B_{1} \quad \cdots \quad \Delta ; \Gamma \rightarrow B_{n}}^{\text {twigs }} i
$$

with label $i$ to be applicable, the init ${ }^{\mathcal{R}}$ set of the conclusion must contain $i$. The bookkeeping rules of an application of rule instance $i$ are as follows.

(a) Copy the bookkeeping sets from the conclusion to each of the premises.

(b) Remove $i$ from the init ${ }^{\mathcal{R}}$ set of each of the premises.

5. For a $\perp \mathrm{L}$ derived rule instance

$$
\overbrace{\Delta ; \Gamma \rightarrow B_{1} \quad \cdots \quad \Delta ; \Gamma \rightarrow B_{n}}^{\text {twigs }} i
$$

with label $i$ to be applicable, the $\perp \mathrm{L}^{\mathcal{R}}$ set of the conclusion must contain $i$. The bookkeeping rules of an application of rule instance $i$ are as follows.

(a) Copy the bookkeeping sets from the conclusion to each of the premises.

(b) Remove $i$ from the $\perp \mathrm{L}^{\mathcal{R}}$ set of each of the premises.

6. For a $\square \mathrm{R}$ rule instance

$$
\frac{\Delta ; \cdot \rightarrow A}{\Delta ; \Gamma \rightarrow \square A} i
$$

with label $i$ to be applicable, the $\square \mathrm{R}^{\mathcal{R}}$ set of the conclusion must contain $i$. The bookkeeping rules of an application of rule instance $i$ are as follows.

(a) Copy the bookkeeping sets from the conclusion to the premise.

(b) Refill the $\vee L^{\mathcal{R}}, \perp \mathrm{L}^{\mathcal{R}}$, and init ${ }^{\mathcal{R}}$ sets of the premise.

(c) Move the contents of the $\square \mathrm{L}^{\mathcal{T}}$ and $\diamond \mathrm{L}^{\mathcal{T}}$ sets of the premise into, respectively, the $\square \mathrm{L}^{\mathcal{R}}$ and $\diamond \mathrm{L}^{\mathcal{R}}$ sets of the premise. 
(d) Remove $i$ from the $\square \mathrm{R}^{\mathcal{R}}$ set of the premise.

7. When applying the rule $\supset R$, the behaviour of the rule is different depending on whether or not the premise of the implication that is the principal formula is contained in the nonmodal context. If it is, then the variant

$$
\frac{\Delta ; \Gamma \rightarrow A_{2}}{\Delta ; \Gamma \rightarrow A_{1} \supset A_{2}} \supset \mathrm{R}_{1} \quad\left(A_{1} \in \Gamma\right)
$$

is applied. If it is not, then the variant

$$
\frac{\Delta ; \Gamma, A_{1} \rightarrow A_{2}}{\Delta ; \Gamma \rightarrow A_{1} \supset A_{2}} \supset \mathrm{R}_{2} \quad\left(A_{1} \notin \Gamma\right)
$$

is applied. In both cases, bookkeeping sets are copied from the conclusion to the premise, but in the case of $\supset \mathrm{R}_{2}$, the following additional bookkeeping steps are required.

(a) Refill the $V L^{\mathcal{R}}, \perp L^{\mathcal{R}}$, and init ${ }^{\mathcal{R}}$ sets of the premise.

(b) Move the contents of the $\square \mathrm{L}^{\mathcal{T}}$ and $\diamond \mathrm{L}^{\mathcal{T}}$ sets of the premise into, respectively, the $\square \mathrm{L}^{\mathcal{R}}$ and $\diamond \mathrm{L}^{\mathcal{R}}$ sets of the premise.

8. The behaviour of the remaining schematic inference rules $\left(\wedge \mathrm{R}, \vee \mathrm{R}_{j}, \diamond \mathrm{R}\right)$ is unchanged from $\mathbf{M J}_{\mathbf{I S} 4}$. In all cases, bookkeeping sets from conclusions are simply copied into premises.

To show that $\mathbf{M} \mathbf{J}_{\mathbf{I S} 4}^{\mathrm{B}}$ provides a decision procedure for $\mathbf{M} \mathbf{J}_{\mathbf{I S} 4}$, we must show that backward proof search in it terminates, and that it is sound and complete with respect to $\mathbf{M} \mathbf{J}_{\mathbf{I S} \mathbf{4}}$.

Theorem 15 (Termination of Backward Proof Search in MJ $\mathrm{IS}_{\mathrm{I}}^{\mathrm{B}}$ ). Backward proof search in $\mathbf{M} \mathbf{J}_{\mathbf{I S} 4}^{\mathbf{B}}$ is guaranteed to terminate.

Proof. Every premise of every rule application is measurably smaller than the conclusion, according to a particular size function defined on $\mathbf{M J} \mathbf{I S}_{\mathbf{I S}}^{\mathrm{B}}$ sequents. In particular, we will associate, with every sequent

$$
\Delta ; \Gamma \rightarrow C \| \square \mathrm{L}^{\mathcal{R}} ; \diamond \mathrm{L}^{\mathcal{R}} ; \mathrm{VL}^{\mathcal{R}} ; \perp \mathrm{L}^{\mathcal{R}} ; \text { init }^{\mathcal{R}} ; \square \mathrm{R}^{\mathcal{R}} ; \square \mathrm{L}^{\mathcal{T}} ; \diamond \mathrm{L}^{\mathcal{T}}
$$

the 10-tuple $\left\langle s_{1}, \ldots, s_{10}\right\rangle$, where $s_{1}, \ldots, s_{10}$ are defined as follows.

$$
\begin{aligned}
s_{1} & =\left|\square \mathrm{L}^{\mathcal{R}} \cup \square \mathrm{L}^{\mathcal{T}}\right| & s_{2} & =\left|\diamond \mathrm{L}^{\mathcal{R}} \cup \diamond \mathrm{L}^{\mathcal{T}}\right| \\
s_{3} & =\left|\square \mathrm{R}^{\mathcal{R}}\right| & s_{4} & =f-|\Gamma| \\
s_{5} & =\left|\square \mathrm{L}^{\mathcal{R}}\right| & s_{6} & =\left|\diamond \mathrm{L}^{\mathcal{R}}\right| \\
s_{7} & =\left|\vee \mathrm{L}^{\mathcal{R}}\right| & s_{8} & =\left|\perp \mathrm{L}^{\mathcal{R}}\right| \\
s_{9} & =\mid \text { init }^{\mathcal{R}} \mid & s_{10} & =\operatorname{size}(C)
\end{aligned}
$$

where $f$ is the number of distinct negative subformula occurrences in $\Delta^{=}, \Gamma^{-}$, and $C^{+},|\Gamma|$ is the number of distinct formula occurrences in the context $\Gamma$, and size $(C)$ is the size of the formula occurrence $C$, computed inductively in the usual way. As in the proof of Lemma $33,\left\langle v_{1}, \ldots, v_{n}\right\rangle\left\langle\left\langle w_{1}, \ldots, w_{n}\right\rangle\right.$ if there is an $i \in\{1, \ldots, n\}$ such that $v_{j}=w_{j}$ for $j<i$ and $v_{i}<w_{i}$. 
Theorem 16 (Soundness of $\mathrm{MJ}_{\mathrm{IS} 4}^{\mathrm{B}}$ with respect to $\mathrm{MJ}_{\mathrm{IS} 4}$ ).

If $\Delta ; \Gamma \rightarrow A$ in $\mathbf{M J}_{\mathbf{I S} 4}^{\mathrm{B}}$, with full $*^{\mathcal{R}}$ and empty $*^{\mathcal{T}}$ sets generated from the query formula $A$, then $\Delta ; \Gamma \rightarrow A$ in $\mathbf{M} \mathbf{J}_{\mathbf{I S} 4}$.

Proof. By induction on the structure of $\mathcal{D}$. The inference rules of $\mathbf{M J}_{\mathbf{I S} 4}^{\mathrm{B}}$ function identically to those of $\mathbf{M J}_{\mathbf{I S} 4}$, only equipped with additional side conditions. This means that the proof space of $\mathbf{M} \mathbf{J}_{\mathbf{I S} 4}^{\mathrm{B}}$ is simply a restricted version of MJ $\mathbf{J}_{\text {IS4 }}$.

\section{Theorem 17 (Completeness of $\mathrm{MJ}_{\mathrm{IS} 4}^{\mathrm{B}}$ with respect to $\mathrm{MJ}_{\mathrm{IS} 4}$ ).}

If $\cdot ; \cdot \stackrel{\mathcal{D}}{\rightarrow} A$ in $\mathbf{M J}_{\mathbf{I S} 4}$, then $; ; \cdot \rightarrow A$ in $\mathbf{M} \mathbf{J}_{\mathbf{I S} 4}^{\mathrm{B}}$, with full $*^{\mathcal{R}}$ and empty $*^{\mathcal{T}}$ sets, generated from the query formula $A$.

Proof. We will show that if an application of a rule in an $\mathbf{M} \mathbf{J}_{\mathbf{I S} 4}$ derivation with derived rules violates a side condition of the corresponding rule in $\mathbf{M J}_{\mathbf{I S} 4}^{\mathrm{B}}$, then the offending application can be removed or modified so as to not violate the side conditions of the corresponding $\mathbf{M} \mathbf{J}_{\mathbf{I S} \mathbf{4}}^{\mathrm{B}}$ rule. Showing that the process of removing such violations terminates then shows that every $\mathbf{M} \mathbf{J}_{\mathbf{I S} 4}$ derivation can be transformed into one without violations, and thus into a valid $\mathbf{M J} \mathbf{I S}_{\mathbf{I S}}^{\mathrm{B}}$ derivation.

Case: An application of the $\square \mathrm{L}$ derived rule instance

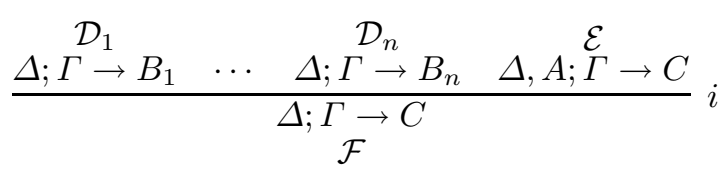

violates a side condition of the corresponding rule in $\mathbf{M} \mathbf{J}_{\mathbf{I S} 4}^{\mathrm{B}}$. Then the $\square \mathrm{L}^{\mathcal{R}}$ set of the conclusion does not contain $i$, so $i$ must have been removed from the branch's $\square \mathrm{L}^{\mathcal{R}}$ set by an application of $i$ in $\mathcal{F}$. Consider the closest such application.

Subcase:

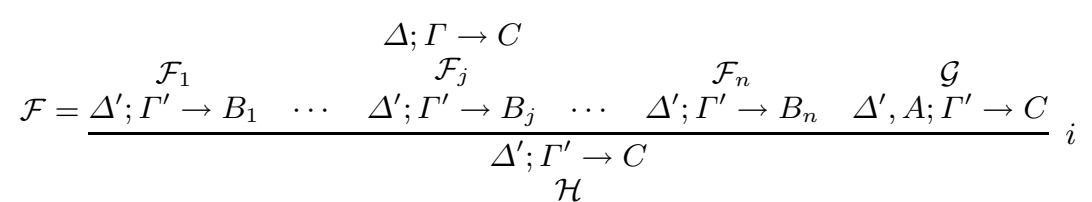

Every rule that changes either context moves the rules in $\square \mathrm{L}^{\mathcal{T}}$ to $\square \mathrm{L}^{\mathcal{R}}$, and the application of $i$ in $\mathcal{F}$ adds $i$ to the $\square \mathrm{L}^{\mathcal{T}}$ set of each twig. So $\mathcal{F}_{j}$ cannot contain any rules that change either context, as otherwise, $i$ would be in the $\square \mathrm{L}^{\mathcal{R}}$ set of the conclusion of the upper application of $i$. So $\Delta^{\prime}=\Delta, \Gamma^{\prime}=\Gamma$, and we can collapse the violating segment into

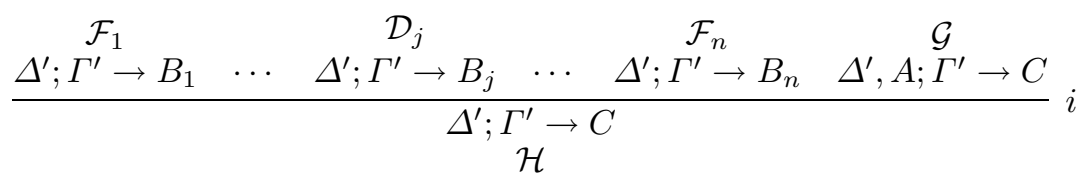




\section{Subcase:}

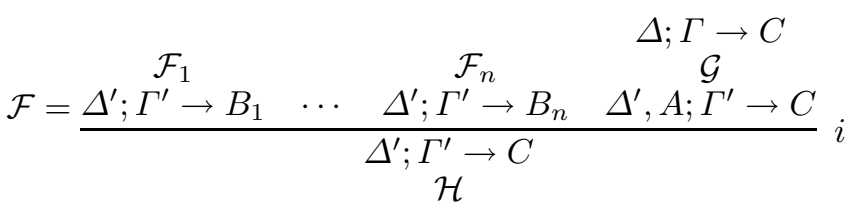

Since no rule reduces the size of the modal context, $A \in \Delta$, so we can collapse the violating segment into

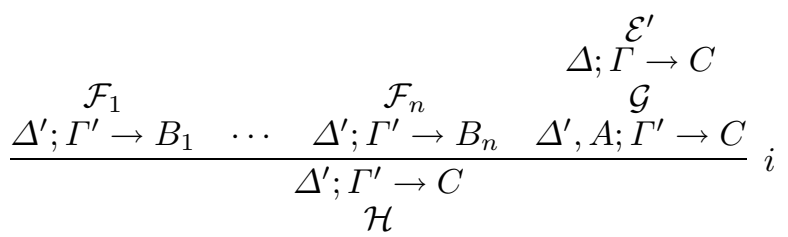

where $\mathcal{E}^{\prime}$ is obtained by applying Lemma 37 to $\mathcal{E}$.

Case: An application of the $\diamond \mathrm{L}$ derived rule instance

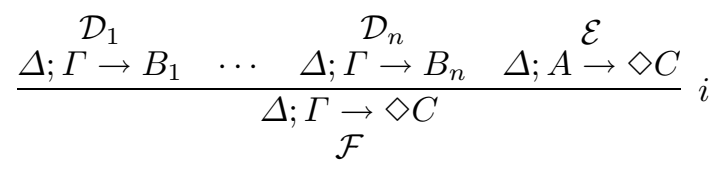

violates a side condition of the corresponding rule in $\mathbf{M} \mathbf{J}_{\text {IS4 }}^{\mathrm{B}}$. Then the $\diamond \mathrm{L}^{\mathcal{R}}$ set of the conclusion does not contain $i$, so $i$ must have been removed from the branch's $\diamond \mathrm{L}^{\mathcal{R}}$ set by an application of $i$ in $\mathcal{F}$. Consider the closest such application.

\section{Subcase:}

$$
\begin{aligned}
& \Delta ; \Gamma \rightarrow \diamond C
\end{aligned}
$$

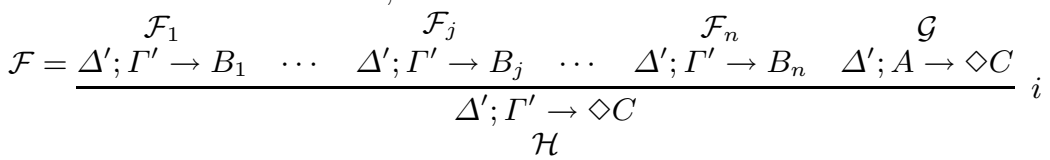

Every rule that changes either context moves the rules in $\diamond \mathrm{L}^{\mathcal{T}}$ to $\diamond \mathrm{L}^{\mathcal{R}}$ or refills $\diamond \mathrm{L}^{\mathcal{R}}$, and the application of $i$ in $\mathcal{F}$ adds $i$ to the $\diamond \mathrm{L}^{\mathcal{T}}$ set of each twig. So $\mathcal{F}_{j}$ cannot contain any rules that change either context, as otherwise, $i$ would be in the $\diamond \mathrm{L}^{\mathcal{R}}$ set of the conclusion of the upper application of $i$. So $\Delta^{\prime}=\Delta, \Gamma^{\prime}=\Gamma$, and we can collapse the violating segment into

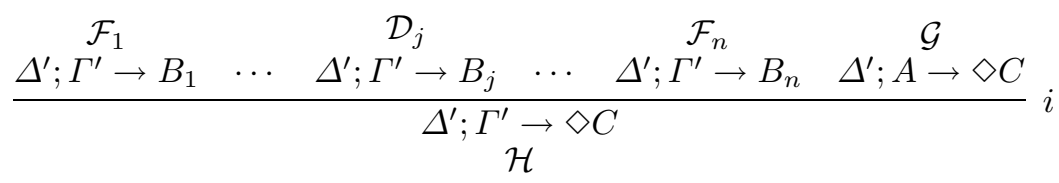




\section{Subcase:}

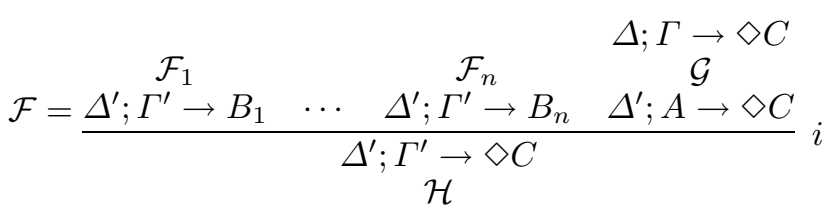

Every rule that changes the modal context refills $\diamond \mathbf{L}^{\mathcal{R}}$, so $\mathcal{G}$ cannot contain any rules that change the modal context, as otherwise, $i$ would be in the $\diamond \mathrm{L}^{\mathcal{R}}$ set of the conclusion of the upper application of $i$. So $\Delta^{\prime}=\Delta$, and we can collapse the violating segment into

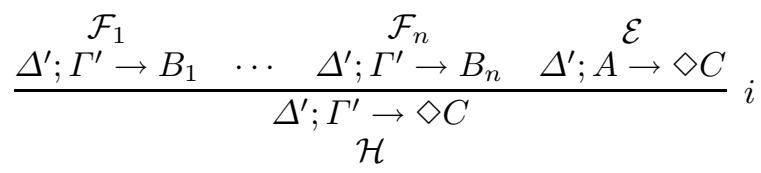

Case: An application of the $V L$ derived rule instance

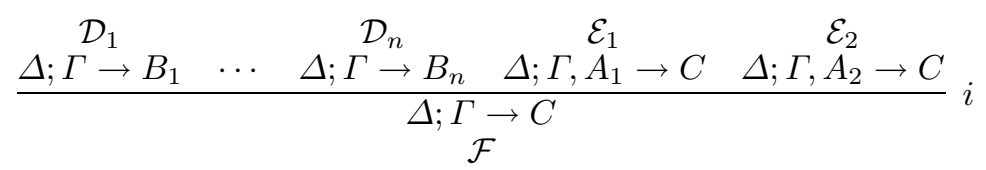

violates a side condition of the corresponding rule in $\mathbf{M} \mathbf{J}_{\mathbf{I S} 4}^{\mathrm{B}}$. Then either

1. $A_{1} \in \Gamma$,

2. $A_{2} \in \Gamma$, or

3. The $\vee \mathrm{L}^{\mathcal{R}}$ set of the conclusion does not contain $i$.

In the first two cases, Lemma 36 can be applied to obtain a derivation of $\Delta ; \Gamma \rightarrow C$ from $\mathcal{E}_{1}$ or $\mathcal{E}_{2}$, eliminating the offending application of $i$. In the third case, $i$ must have been removed from the branch's $\vee L^{\mathcal{R}}$ set by an application of $i$ in $\mathcal{F}$. Consider the closest such application.

\section{Subcase:}

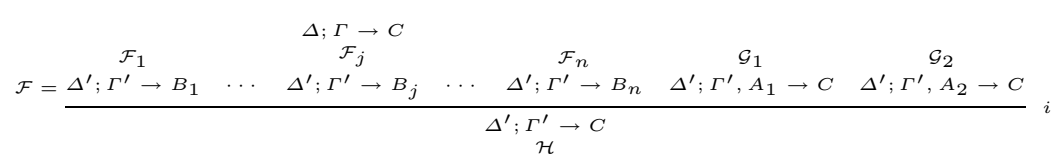

Every rule that changes either context refills $\vee L^{\mathcal{R}}$, so $\mathcal{F}_{j}$ cannot contain any rules that change either context, as otherwise, $i$ would be in the $\mathrm{VL}^{\mathcal{R}}$ set of the conclusion of the upper application of $i$. So $\Delta^{\prime}=\Delta, \Gamma^{\prime}=\Gamma$, and we can collapse the violating segment into

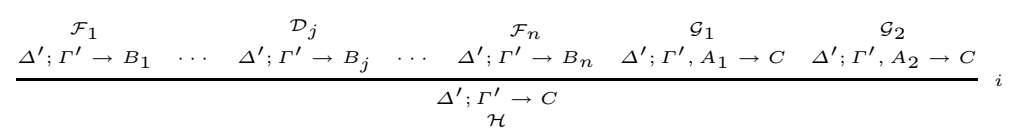




\section{Subcase:}

$$
\begin{aligned}
& \Delta ; \Gamma \rightarrow C
\end{aligned}
$$

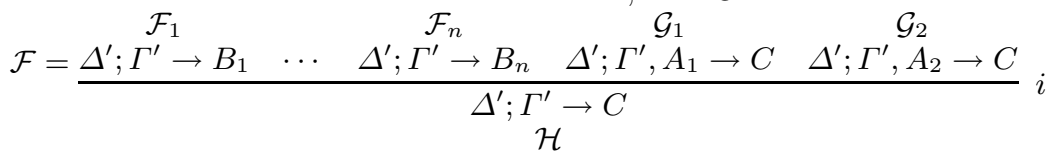

Every rule that changes either context refills $\vee L^{\mathcal{R}}$, so $\mathcal{G}_{1}$ cannot contain any rules that change either context, as otherwise, $i$ would be in the $\mathrm{VL}^{\mathcal{R}}$ set of the conclusion of the upper application of $i$. So $\Delta^{\prime}=\Delta$, $\Gamma^{\prime}, A_{1}=\Gamma$, and we can collapse the violating segment into

$$
\begin{aligned}
& \begin{array}{c}
\mathcal{E}_{1}^{\prime} \\
\Delta ; \Gamma \rightarrow C
\end{array}
\end{aligned}
$$

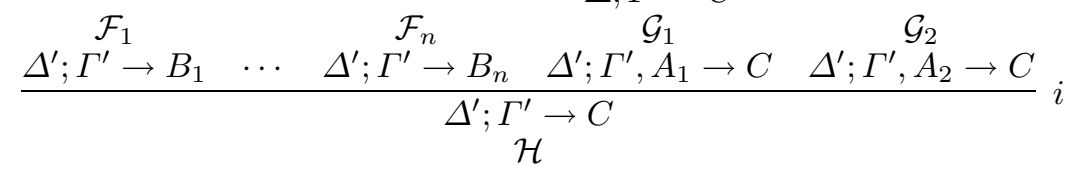

where $\mathcal{E}_{1}^{\prime}$ is obtained by applying Lemma 36 to $\mathcal{E}_{1}$.

\section{Subcase:}

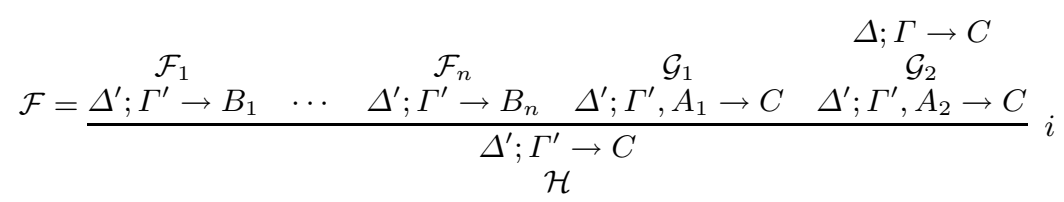

Every rule that changes either context refills $\vee L^{\mathcal{R}}$, so $\mathcal{G}_{2}$ cannot contain any rules that change either context, as otherwise, $i$ would be in the $\mathrm{VL}^{\mathcal{R}}$ set of the conclusion of the upper application of $i$. So $\Delta^{\prime}=\Delta$, $\Gamma^{\prime}, A_{2}=\Gamma$, and we can collapse the violating segment into

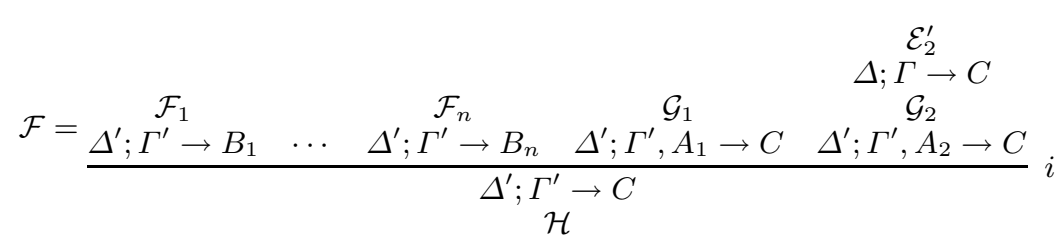

where $\mathcal{E}_{2}^{\prime}$ is obtained by applying Lemma 36 to $\mathcal{E}_{2}$.

Case: An application of the $\perp \mathrm{L}$ derived rule instance

$$
\frac{\stackrel{\mathcal{D}_{1}}{\Delta ; \Gamma \stackrel{ }{\rightarrow} B_{1} \quad \cdots \quad} \stackrel{\mathcal{D}_{n}}{\rightarrow} \quad \Delta ; \Gamma^{\rightarrow} B_{n}}{\Delta ; \Gamma_{\mathcal{F}}^{\rightarrow} C} i
$$

violates a side condition of the corresponding rule in $\mathbf{M} \mathbf{J}_{\mathbf{I S} 4}^{\mathrm{B}}$. Then the $\perp \mathrm{L}^{\mathcal{R}}$ set of the conclusion does not contain $i$, so $i$ must have been removed from 
the branch's $\perp \mathrm{L}^{\mathcal{R}}$ set by an application of $i$ in $\mathcal{F}$. Consider the closest such application.

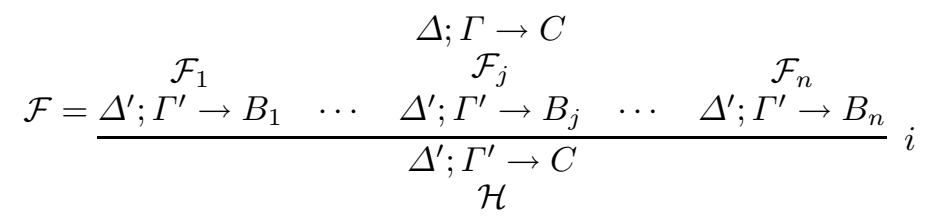

Every rule that changes either context refills $\perp \mathrm{L}^{\mathcal{R}}$, so $\mathcal{F}_{j}$ cannot contain any rules that change either context, as otherwise, $i$ would be in the $\perp \mathrm{L}^{\mathcal{R}}$ set of the conclusion of the upper application of $i$. So $\Delta^{\prime}=\Delta, \Gamma^{\prime}=\Gamma$, and we can collapse the violating segment into

$$
\begin{array}{ccccc}
\mathcal{F}_{1} & \mathcal{D}_{j} & \mathcal{F}_{n} \\
\Delta^{\prime} ; \Gamma^{\prime} \rightarrow B_{1} & \cdots & \Delta^{\prime} ; \Gamma^{\prime} \rightarrow B_{j} & \cdots & \Delta^{\prime} ; \Gamma^{\prime} \rightarrow B_{n} \\
\hline \multicolumn{4}{c}{\Delta^{\prime} ; \Gamma^{\prime} \rightarrow C}
\end{array}
$$

Case: An application of the init derived rule instance

$$
\frac{\stackrel{\mathcal{D}_{1}}{\Delta ; \Gamma \stackrel{\mathcal{D}_{n}}{\rightarrow} B_{1} \quad \cdots \quad \Delta ; \Gamma^{\rightarrow} B_{n}}}{\Delta ; \Gamma_{\mathcal{F}}^{\rightarrow} C} i
$$

violates a side condition of the corresponding rule in $\mathbf{M} \mathbf{J}_{\mathbf{I S} 4}^{\mathrm{B}}$. Then the init ${ }^{\mathcal{R}}$ set of the conclusion does not contain $i$, so $i$ must have been removed from the branch's init ${ }^{\mathcal{R}}$ set by an application of $i$ in $\mathcal{F}$. Consider the closest such application.

$$
\begin{aligned}
& \Delta ; \Gamma \rightarrow C
\end{aligned}
$$

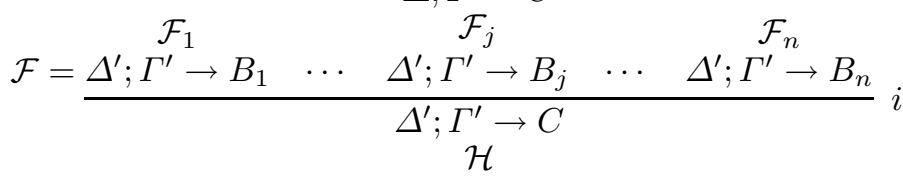

Every rule that changes either context refills init ${ }^{\mathcal{R}}$, so $\mathcal{F}_{j}$ cannot contain any rules that change either context, as otherwise, $i$ would be in the init ${ }^{\mathcal{R}}$ set of the conclusion of the upper application of $i$. So $\Delta^{\prime}=\Delta, \Gamma^{\prime}=\Gamma$, and we can collapse the violating segment into

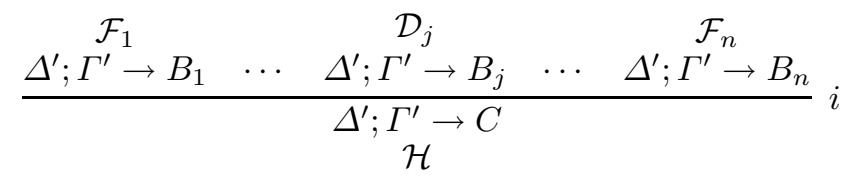

Case: An application of the $\square \mathrm{R}$ rule instance

$$
\underset{\mathcal{F}}{\frac{\mathcal{D}_{1}}{\Delta ;} \rightarrow \square A} \rightarrow
$$


violates a side condition of the corresponding rule in $\mathbf{M} \mathbf{J}_{\mathbf{I S} 4}^{\mathbf{B}}$. Then the $\square \mathrm{R}^{\mathcal{R}}$ set of the conclusion does not contain $i$, so $i$ must have been removed from the branch's $\square \mathrm{R}^{\mathcal{R}}$ set by an application of $i$ in $\mathcal{F}$. Consider the closest such application.

$$
\begin{gathered}
\Delta ; \Gamma \rightarrow A \\
\mathcal{F}=\frac{\mathcal{F}_{1} ; \cdot \rightarrow \square A}{\Delta^{\prime} ; \Gamma_{\mathcal{H}}^{\prime} \rightarrow A} i
\end{gathered}
$$

Every rule that changes the modal context refills $\square \mathrm{R}^{\mathcal{R}}$, so $\mathcal{F}_{1}$ cannot contain any rules that change the modal context, as otherwise, $i$ would be in the $\square \mathrm{R}^{\mathcal{R}}$ set of the conclusion of the upper application of $i$. So $\Delta^{\prime}=\Delta$, and we can collapse the violating segment into

$$
\frac{\Delta^{\prime} ; \cdot \stackrel{\mathcal{D}_{1}}{\rightarrow} \square A}{\Delta^{\prime} ; \Gamma^{\prime} \rightarrow A} i
$$

Applications of schematic rules in $\mathbf{M} \mathbf{J}_{\mathbf{I S} 4}^{\mathbf{B}}$ have no side conditions, and can thus not cause any violations. Each conversion shown above eliminates one violating application of a rule instance application from an $\mathbf{M} \mathbf{J}_{\mathbf{I S} 4}$ derivation, and since there can be only finitely many violations in such a derivation, the procedure above shows how to obtain an $\mathbf{M} \mathbf{J}_{\mathbf{I S} 4}^{\mathbf{B}}$ derivation from an $\mathbf{M} \mathbf{J}_{\mathbf{I S} 4}$ derivation.

These results show that $\mathbf{M} \mathbf{J}_{\mathbf{I S} 4}^{\mathbf{B}}$ is a sound and complete decision procedure for propositional IS4. Although the bookkeeping needed to guarantee termination and completeness is slightly cumbersome, it turns out that it is rarely necessary. If a query formula is provable, then in almost all cases, simply restricting the number of derived rule instances used along a branch is a sufficiently lax condition to allow a proof to be found. Only if a proof is not found is it necessary to perform some bookkeeping to guarantee that the search space has been exhausted.

Note that the idea of constructing relevant derived rules prior to proof search can also be exploited in forward proof search, where the derived rules described above can take the place of left rules in the inverse method. The main advantages here are that the derived rules are more relevant to proof search for the given query formula, and that the number of intermediate sequents added to the knowledge base during proof search is reduced, since no focused sequents need to be maintained. Naturally, in a forward direction, no bookkeeping is required. Instead, proof space saturation needs to be monitored to identify when to terminate parts of the search.

\section{Bidirectional Proof Search in Natural Deduction}

In the bidirectional sequent calculus method, we construct derived rules to conceal all required focused threads. Notice that the focused threads of $\mathbf{M} \mathbf{J}_{\mathbf{I S 4}}^{\mathbf{F}}$ 
correspond naturally to segments of $\mathbf{N J}_{\mathbf{I S} 4}^{\mathbf{N}}$ proofs consisting of elimination rule applications, that is, $\downarrow$ judgements. The beginnings of focused threads, where formulas are placed into the stoup, correspond to reversing rules in $\mathbf{N J}_{\mathbf{I S} 4}^{\mathbf{N}}$. These are the $\uparrow \downarrow$ rule, as well as all elimination rules with $\uparrow$ judgements as their conclusions. The ends of focused threads, on the other hand, where the stoup formula is dropped into a context, correspond to using a hypothesis with applications of hyp $_{1}$ or hyp . $_{2}$.

This means that the process of building a derived $\mathbf{M} \mathbf{J}_{\mathbf{I S} 4}^{\mathbf{F}}$ rule in a top-down way corresponds to building a natural deduction derived rule by beginning with an application of a reversing rule, and growing it upwards until we reach a leaf. Just as the construction of derived rules in the sequent calculus is determined uniquely by the form of the query formula, so these natural deduction derived rules can be deterministically constructed before proof search even begins.

This approach is best demonstrated by an example such as the one given in Sect. 7. For instance, given the pair $L_{4} \approx$ and $L_{8}^{+}$from that example, we begin with the coercion

$$
\frac{\Delta^{=} ; \Gamma^{-} \vdash L \widetilde{\tilde{4}} \downarrow}{\Delta^{=} ; \Gamma^{-} \vdash L_{8}^{+} \uparrow} \uparrow \downarrow
$$

Since the immediate parent of $L \approx \widetilde{4}$ in the signed subformula tree is $L \approx \widetilde{2}$, denoting $A \supset B$, the rule application above this coercion must be an application of $\supset \mathrm{E}$ :

$$
\frac{\Delta^{=} ; \Gamma^{-} \vdash L_{2} \approx \downarrow \quad \Delta^{=} ; \Gamma^{-} \vdash L_{3}^{+} \uparrow}{\frac{\Delta^{=} ; \Gamma^{-} \vdash L_{4}}{\Delta^{=} ;} \uparrow \Gamma^{-} \vdash L_{8}^{+} \uparrow} \uparrow \mathrm{E}
$$

The focused thread continues along the first premise, but the parent of $L_{2} \approx$ is $L_{2}^{\bar{z}}$, indicating the end of this focused thread by an application of hyp $\mathrm{p}_{2}$ :

$$
\frac{\frac{L_{2}^{=} \in \Delta^{=}}{\Delta^{=} ; \Gamma^{-} \vdash L_{2} \downarrow} \operatorname{hyp}_{2} \quad \Delta^{=} ; \Gamma^{-} \vdash L_{3}^{+} \uparrow}{\frac{\Delta^{=} ; \Gamma^{-} \vdash L_{4} \downarrow}{\Delta^{=} ; \Gamma^{-} \vdash L_{8}^{+} \uparrow} \uparrow \downarrow} \supset \mathrm{E} \leadsto \frac{\Delta_{1}^{=}, L_{2}^{=}, \Delta_{2}^{=} ; \Gamma^{-} \vdash L_{3}^{+} \uparrow}{\Delta_{1}^{=}, L_{2}^{=}, \Delta_{2}^{=} ; \Gamma^{-} \vdash L_{8}^{+} \uparrow}
$$

In similar constructions, the pair $L_{7}^{\tilde{7}}, L_{3}^{+}$and $L_{1}^{\sim}$, the latter denoting $\square(A \supset B)$, produce, respectively, the natural deduction derived rules

$$
\frac{\frac{L_{7}^{-} \in \Gamma^{-}}{\Delta^{=} ; \Gamma^{-} \vdash L_{7}^{\tilde{}} \downarrow}}{\Delta^{=} ; \Gamma^{-} \vdash L_{3}^{+} \uparrow} \operatorname{hyp}_{1} \downarrow \leadsto \frac{}{\Delta^{=} ; \Gamma_{1}^{-}, L_{7}^{-}, \Gamma_{2}^{-} \vdash L_{3}^{+} \uparrow}
$$

and

$$
\frac{\frac{L_{1}^{-} \in \Gamma^{-}}{\Delta^{=} ; \Gamma^{-} \vdash L_{1}^{\sim} \downarrow} \operatorname{hyp}_{1} \quad \Delta^{=}, L_{2}^{=} ; \Gamma^{-} \vdash M^{+} \uparrow}{\Delta^{=} ; \Gamma^{-} \vdash M^{+} \uparrow} \square \mathrm{E}
$$




$$
\leadsto \quad \frac{\Delta^{=}, L_{2}^{=} ; \Gamma_{1}^{-}, L_{1}^{-}, \Gamma_{2}^{-} \vdash M^{+} \uparrow}{\Delta^{=} ; \Gamma_{1}^{-}, L_{1}^{-}, \Gamma_{2}^{-} \vdash M^{+} \uparrow}(3)
$$

The rest of the proof then uses only these derived rules and introduction rules:

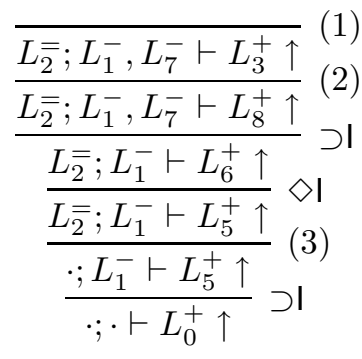

In general, the approach for constructing natural deduction derived rules is analogous to the method for the bidirectional sequent calculus, only turned upsidedown, in the sense that the rule at the beginning of an $\mathbf{M} \mathbf{J}_{\mathbf{I S} 4}^{\mathbf{F}}$ focused thread determines the reversing rule at the bottom of the natural deduction focused thread, while the final application of hyp ${ }_{i}$ dictates the "principal formula" of the ensuing derived natural deduction rule.

Proof search over natural deductions can then be performed in a backward direction. The only nondeterminism is in whether to apply a derived rule or an introduction rule, the premises of which are uniquely determined by their conclusions. Note that to guarantee termination, we can again restrict or incrementally increase the number of allowed derived rule instances along a branch of a proof. Sound and complete bookkeeping systems equivalent to the one presented in Sect. 7 can also be designed for the natural deduction setting.

\section{$9 \quad$ Experimental Results}

While benchmark formulas are available for intuitionistic propositional logic and classical modal logics, we are not aware of any benchmark libraries specific to propositional IS4. In order to evaluate the performance of our bidirectional approach, we put together a benchmark set of 50 formulas for IS4, mostly problems from Raths et al.'s Intuitionistic Logic Theorem Proving (ILTP) library [18] to which we introduced modalities. Our full benchmark set is given in Appendix A.

We implemented three IS4 decision procedures in SML: (1) an $\mathbf{M J}_{\mathbf{I S}} \mathbf{4}_{\text {-based }}$ backward prover with a conventional history mechanism for loop-detection, (2) an $\mathbf{M} \mathbf{J}_{\mathbf{I S} 4}^{\mathbf{F}}$-based inverse method prover without derived rules, and (3) our bidirectional natural deduction prover. The loop-detection prover maintains two histories to detect repeated modal and nonmodal rule applications, respectively. This approach is a generalization of Howe's decision procedure [12] extended to full IS4. Note that the behaviour of our bidirectional sequent calculus prover corresponds exactly to that of the bidirectional natural deduction prover, so we have only implemented the more elegant natural deduction prover. In the bidirectional prover, we first restrict the number of derived rule instances to at most 
Table 1. Selection of experimental results

\begin{tabular}{ccccccccc}
\hline & & & & Histories & \multicolumn{2}{c}{ Inverse } & \multicolumn{2}{c}{ Bidirectional } \\
Formula & Size & Modalities & Provable & Time & Time & Rules & Time & Rules \\
\hline 32 & 49 & 0 & N & $>1000$ & 1.36 & 33 & 0.01 & 33 \\
36 & 175 & 0 & Y & 0.08 & $>1000$ & 159 & $>1000$ & 592 \\
37 & 68 & 9 & Y & 84.79 & 1.18 & 60 & $<0.01$ & 28 \\
39 & 42 & 3 & N & 8.46 & 1.83 & 31 & $<0.01$ & 15 \\
44 & 49 & 14 & Y & 75.13 & $>1000$ & 51 & 37.11 & 21 \\
50 & 44 & 7 & Y & 7.38 & $>1000$ & 49 & 48.76 & 25 \\
\hline
\end{tabular}

one schematic derived rule per branch, then relax this constraint if no proof is found. In most cases, this allows proofs to be found significantly faster, if they exist. In fact, this approach makes much of the bookkeeping presented in Sect. 7 redundant if the query formula is provable.

On many of the smaller problems, there was little measurable difference in the performance of the provers, but some of the problems that did elicit noticeably different performances are highlighted in Table 1 . The size column shows the complexity of each formula, computed inductively in the usual way, while the modalities column shows the number of modal operators. Times are in seconds. ${ }^{3}$ For the inverse method and bidirectional provers, we show the number of inference rules generated (derived rules in the case of the bidirectional prover).

As the results demonstrate, the bidirectional natural deduction prover is a competitive alternative to the more conventional provers, equalling or outperforming them on most problems. Comparing the average proving time for problems that were solved, it is noticeably superior, although we found two formulas on which it was significantly outperformed (formulas 36 and 50 in Table 1). Interestingly, there is not always a clear connection between the number of derived rules generated and the time required to solve a problem. Presumably, the problematic cases were those whose derived rules were the shortest and least useful. Note also that as derived rules are associated with subformula occurrences, those formulas with many repeated subformulas (e.g. formula 36) caused a very large number of duplicate derived rules to be generated.

\section{Related and Future Work}

Although IS4 has undergone thorough proof-theoretical studies, there has been little work in developing proof search strategies specific to it. We have presented a comprehensive study of proof search formalisms for IS4, highlighting the duality between backward and forward search. Moreover, we have demonstrated how to combine the benefits of both to yield bidirectional decision procedures based

\footnotetext{
${ }^{3}$ All timing results were obtained on a Pentium III $850 \mathrm{MHz}$ with $256 \mathrm{MB}$ of RAM, running SML/NJ version 110.60 .
} 
on sequent calculi and natural deduction. Our experimental results reveal that combining the two traditionally disparate paradigms can be fruitful. Although our implementations are naive and unoptimized, we hope that our results might encourage further study of bidirectional proof search, particularly in other logics.

For instance, in the contextual modal logic of Nanevski, Pfenning, and Pientka [15], structural modality is generalized by relativizing the validity judgement and the modal operators. The techniques discussed in this paper extend very naturally to contextual modal logic, yielding sequent calculi suitable for backward and forward proof search, but the exact nature of how such a generalization affects proof search is yet to be explored. The reconciliation of forward and backward proof search has recently also been investigated by Chaudhuri and Pfenning [3], who, in the context of linear logic, propose a focusing inverse method prover incorporating derived rules constructed in a backward way and searched over in a forward direction, precisely opposite to our approach.

In the future, we plan to explore extensions to the first-order case. Although the idea of derived rules extends, in principle, to first-order quantifiers, the constructed derived rules become parametric in terms. The characterization of a derived rule instance now also includes the parameter instantiations, introducing another layer of bookkeeping. How to efficiently overcome this problem and what the proof-theoretical relationship between first-order bidirectional decision procedures and natural deduction provers is remains to be investigated.

Acknowledgements. We would like to thank Daniel Pomerantz for discussions on forward proof search and for the implementation of the inverse method prover, and the reviewers of the conference version of this paper [11] for their insightful comments and suggestions.

\section{References}

1. N. Alechina, M. Mendler, V. de Paiva, and E. Ritter. Categorical and Kripke semantics for constructive S4 modal logic. In L. Fribourg (ed.) Proceedings of the 15th International Workshop on Computer Science Logic, pp. 292-307. Springer LNCS 2142, 2001.

2. G. M. Bierman and V. C. V. de Paiva. On an intuitionistic modal logic. Studia Logica, 65(3):383-416, 2000.

3. K. Chaudhuri and F. Pfenning. Focusing the inverse method for linear logic. In C.-H. L. Ong (ed.) Proceedings of the 19th International Workshop on Computer Science Logic, 14th Annual Conference of the EACSL, pp. 200-215. Springer LNCS 3634, 2005.

4. R. Davies and F. Pfenning. A modal analysis of staged computation. Journal of the ACM, 48(3):555-604, 2001.

5. A. Degtyarev and A. Voronkov. The inverse method. In J. A. Robinson and A. Voronkov (eds.) Handbook of Automated Reasoning, pp. 179-272. Elsevier and MIT Press, 2001.

6. R. Dyckhoff and L. Pinto. A permutation-free sequent calculus for intuitionistic logic. Research Report CS/96/9, University of St. Andrews, 1996. 
7. M. Fairtlough and M. Mendler. Propositional lax logic. Information and Computation, 137(1):1-33, 1997.

8. F. B. Fitch. Intuitionistic modal logic with quantifiers. Portugaliae Mathematica, $7(2): 113-118,1948$.

9. J.-Y. Girard. A new constructive logic: Classical logic. Mathematical Structures in Computer Science, 1(3):255-296, 1991.

10. J.-Y. Girard, Y. Lafont, and P. Taylor. Proofs and types. Cambridge University Press, 1989.

11. S. Heilala and B. Pientka. Bidirectional decision procedures for the intuitionistic propositional modal logic IS4. In F. Pfenning (ed.) Proceedings of the 21st International Conference on Automated Deduction, pp. 116-131. Springer LNAI 4603, 2007.

12. J. M. Howe. Proof search issues in some non-classical logics. PhD thesis, University of St. Andrews, 1998.

13. J. M. Howe. Proof search in lax logic. Mathematical Structures in Computer Science, 11(4): 573-588, 2001.

14. J. Moody. Modal logic as a basis for distributed computation. Technical Report CMU-CS-03-194, Carnegie Mellon University, 2003.

15. A. Nanevski, F. Pfenning, and B. Pientka. Contextual modal type theory. To appear in ACM Transactions on Computational Logic.

16. F. Pfenning and R. Davies. A judgmental reconstruction of modal logic. Mathematical Structures in Computer Science, 11(4):511-540, 2001.

17. D. Prawitz. Natural deduction: A proof-theoretical study. Almqvist \& Wiksell, 1965.

18. T. Raths, J. Otten, and C. Kreitz. The ILTP problem library for intuitionistic logic, release v1.1. To appear in Journal of Automated Reasoning.

19. T. W. Satre. Natural deduction rules for modal logics. Notre Dame Journal of Formal Logic, 13(4):461-475, 1972.

20. A. K. Simpson. The proof theory and semantics of intuitionistic modal logic. PhD thesis, University of Edinburgh, 1994.

21. A. S. Troelstra and H. Schwichtenberg. Basic Proof Theory, Second Edition. Cambridge University Press, 2000. 


\section{A Benchmark Formulas}

Our 50 benchmark formulas are shown below. The equivalence operator $\equiv$, used in some of the formulas, is defined notationally in the usual way:

$$
A_{1} \equiv A_{2} \stackrel{\text { def }}{=}\left(A_{1} \supset A_{2}\right) \wedge\left(A_{2} \supset A_{1}\right)
$$

Its precedence is lower than that of all other connectives.

1. $(A \vee B) \vee C \equiv(C \vee B) \vee A$

2. $\square A \vee(\square B \vee \square C) \supset \diamond C \vee(\diamond B \vee \diamond A)$

3. $A \wedge(B \wedge C) \equiv(C \wedge A) \wedge B$

4. $\diamond A \wedge(\square B \vee C) \equiv(\diamond A \wedge \square B) \vee(\diamond A \wedge C)$

5. $(\square(A \supset B)) \supset((\square A) \supset(\square B))$

6. $(\square A) \supset A$

7. $(\square A) \supset(\square(\square A))$

8. $A \supset(\diamond A)$

9. $(\diamond(\diamond A)) \supset(\diamond A)$

10. $(\square(A \supset B)) \supset((\diamond A) \supset(\diamond B))$

11. $(((\square C) \supset E) \supset A) \supset(((\square C) \supset E) \supset A)$

12. $(((\diamond D) \supset E) \supset B) \supset(((\diamond D) \supset E) \supset B)$

13. $(\square(\diamond A)) \supset(\square(\diamond A))$

14. $(\square A) \supset(\diamond A)$

15. $(\diamond A) \supset(\square(\diamond A))$ (not provable)

16. $(\square(\diamond A)) \supset(\diamond(\square A))$ (not provable)

17. $(\diamond(\square A)) \supset(\square(\diamond A))$ (not provable)

18. $A \supset(\square(\diamond A))$ (not provable)

19. $(\square(A \supset(\diamond B))) \supset((\diamond A) \supset(\diamond B))$

20. $(\diamond A) \supset(((\diamond A) \supset(\diamond B)) \supset(\diamond B))$

21. $\square(((\square A) \supset(\square B)) \supset(\square(C \supset(\square D))))) \supset(((\square A) \supset(\square B)) \supset(\square(C \supset$ $(\square D))))$

22. $((b \equiv c) \supset(a \wedge b \wedge c)) \wedge((c \equiv a) \supset(a \wedge b \wedge c)) \wedge((a \equiv b) \supset(a \wedge b \wedge c)) \supset(a \wedge b \wedge c)$

23. $\neg \neg\left(\neg p_{1} \vee \neg p_{2} \vee \neg p_{3} \vee\left(p_{1} \wedge p_{2} \wedge p_{3}\right)\right)$

24. $((a 1 \equiv a 2) \equiv a 3) \equiv(a 3 \equiv(a 2 \equiv a 1))$

25. $\neg\left(p_{1} \supset p_{2}\right) \supset\left(p_{2} \supset p_{1}\right)$

26. $\left(p_{1} \supset p_{2}\right) \wedge\left(p_{2} \supset\left(p_{3} \wedge p_{1}\right)\right) \wedge\left(p_{3} \supset\left(p_{1} \vee p_{2}\right)\right) \supset\left(p_{3} \equiv p_{1}\right)$

27. $\left(p_{1} \vee\left(p_{2} \wedge p_{3}\right)\right) \equiv\left(\left(p_{1} \vee p_{2}\right) \wedge\left(p_{1} \vee p_{3}\right)\right)$

28. $\neg \neg\left(p_{1} \vee \neg p_{1}\right)$

29. $\neg \neg\left(\left(p_{1} \wedge p_{2}\right) \vee\left(\neg p_{1} \vee \neg p_{2}\right)\right)$

30. $\neg \neg\left(\left(p_{1} \wedge\left(p_{2} \wedge p_{3}\right)\right) \vee\left(\neg p_{1} \vee\left(\neg p_{2} \vee \neg p_{3}\right)\right)\right)$

31. $\left(\left(p_{1} \supset p_{2}\right)\right) \wedge\left(\left(\left(\left(\neg\left(\neg p_{3}\right)\right) \supset p_{4}\right) \supset p_{5}\right)\right) \wedge\left(\left(\left(\left(\neg\left(\neg p_{4}\right)\right) \supset p_{5}\right) \supset p_{1}\right)\right) \supset\left(p_{2}\right)$ (not provable)

32. $\left(\left(\neg\left(\neg p_{1}\right)\right)\right) \wedge\left(\left(p_{2} \supset\left(p_{2} \supset p_{3}\right)\right)\right) \wedge\left(\left(p_{4} \supset\left(p_{4} \supset p_{2}\right)\right)\right) \wedge\left(\left(p_{5} \supset\left(p_{5} \supset p_{4}\right)\right)\right) \wedge$ $\left(\left(p_{6} \supset\left(p_{6} \supset p_{5}\right)\right)\right) \wedge\left(\left(p_{7} \supset\left(p_{7} \supset p_{6}\right)\right)\right) \wedge\left(\left(p_{8} \supset\left(p_{8} \supset p_{7}\right)\right)\right) \wedge\left(\left(p_{1} \supset\left(p_{1} \supset\right.\right.\right.$ $\left.\left.\left.p_{8}\right)\right)\right) \supset\left(p_{3}\right)($ not provable $)$

33. $\left(\left(\neg\left(\neg p_{1}\right)\right)\right) \wedge\left(\left(p_{1} \supset\left(p_{1} \supset p_{2}\right)\right)\right) \supset\left(p_{2}\right)$ (not provable)

34. $\left(\left(\neg \diamond\left(\neg \square p_{1}\right)\right)\right) \wedge\left(\left(\neg\left(\neg \square \square p_{2}\right)\right)\right) \supset\left(\left(\diamond p_{1} \vee \diamond p_{2}\right)\right)$ (not provable) 
35. $\left(\left(\left(\square p_{1} \equiv p_{2}\right) \supset\left(p_{1} \wedge p_{2}\right)\right)\right) \wedge\left(\left(\left(p_{2} \equiv \square p_{1}\right) \supset\left(p_{1} \wedge p_{2}\right)\right)\right) \supset\left(\left(p_{3} \vee\left(\left(p_{1} \wedge p_{2}\right) \vee\right.\right.\right.$ $\left.\left.\left(\neg p_{3}\right)\right)\right)$ (not provable)

36. $\left.\left(\left(\left(\left(p_{1} \equiv p_{2}\right) \equiv p_{3}\right) \equiv p_{4}\right) \equiv\left(p_{4} \equiv\left(p_{3} \equiv\left(p_{2} \equiv p_{1}\right)\right)\right)\right)\right)$

37. $\left(\left(\left(\left(\left(\diamond p_{1} \supset p_{2}\right) \wedge\left(\left(\left(\diamond p_{3} \supset \diamond p_{4}\right) \supset p_{5}\right) \wedge\left(\left(\left(\diamond p_{4} \supset \diamond p_{6}\right) \supset p_{1}\right) \wedge\left(\left(\left(\diamond p_{7} \supset\right.\right.\right.\right.\right.\right.\right.\right.\right.$ $\left.\left.\left.\left.\left.\left.\left.\diamond p_{8}\right) \supset p_{6}\right) \wedge\left(\left(p_{9} \supset p_{5}\right) \supset p_{8}\right)\right)\right)\right)\right) \supset p_{2}\right) \wedge\left(\left(\left(\left(p_{9} \supset p_{5}\right) \supset p_{8}\right) \wedge\left(\left(\left(\diamond p_{7} \supset\right.\right.\right.\right.\right.$ $\left.\left.\left.\left.\left.\left.\diamond p_{8}\right) \supset p_{6}\right) \wedge\left(\left(\left(p_{4} \supset p_{6}\right) \supset p_{1}\right) \wedge\left(\left(\left(p_{3} \supset p_{4}\right) \supset p_{5}\right) \wedge\left(p_{1} \supset p_{2}\right)\right)\right)\right) \supset p_{2}\right)\right)\right)$

38. $\left(\left(\left(\left(\left(p_{1} \supset p_{2}\right) \wedge\left(\left(\left(p_{3} \supset p_{4}\right) \supset p_{5}\right) \wedge\left(\left(\left(p_{4} \supset p_{6}\right) \supset p_{1}\right) \wedge\left(\left(\left(p_{7} \supset p_{8}\right) \supset\right.\right.\right.\right.\right.\right.\right.\right.$ $\left.\left.\left.\left.\left.\left.p_{6}\right) \wedge\left(\left(\left(p_{9} \supset p_{10}\right) \supset p_{8}\right) \wedge\left(\left(p_{11} \supset p_{5}\right) \supset p_{10}\right)\right)\right)\right)\right)\right) \supset p_{2}\right) \wedge\left(\left(\left(\left(p_{11} \supset p_{5}\right) \supset\right.\right.\right.$ $\left.p_{10}\right) \wedge\left(\left(\left(p_{9} \supset p_{10}\right) \supset p_{8}\right) \wedge\left(\left(\left(p_{7} \supset p_{8}\right) \supset p_{6}\right) \wedge\left(\left(\left(p_{4} \supset p_{6}\right) \supset p_{1}\right) \wedge\left(\left(\left(p_{3} \supset\right.\right.\right.\right.\right.\right.$ $\left.\left.\left.\left.\left.\left.\left.\left.\left.\left.p_{4}\right) \supset p_{5}\right) \wedge\left(p_{1} \supset p_{2}\right)\right)\right)\right)\right)\right) \supset p_{2}\right)\right)\right)$

39. $\diamond\left(p_{1}\right) \wedge \square\left(\left(p_{2} \supset\left(p_{2} \supset p_{3}\right)\right)\right) \wedge\left(\left(p_{4} \supset\left(p_{4} \supset p_{2}\right)\right)\right) \wedge\left(\left(p_{5} \supset\left(p_{5} \supset p_{4}\right)\right)\right) \wedge\left(\left(p_{6} \supset\right.\right.$ $\left.\left.\left(p_{6} \supset p_{5}\right)\right)\right) \wedge\left(\left(p_{7} \supset\left(p_{7} \supset p_{6}\right)\right)\right) \wedge\left(\left(p_{1} \supset\left(p_{1} \supset p_{7}\right)\right)\right) \supset \diamond\left(p_{3}\right)$ (not provable)

40. $\left(p_{1}\right) \wedge\left(\left(p_{2} \supset\left(p_{2} \supset p_{3}\right)\right)\right) \wedge\left(\left(p_{4} \supset\left(p_{4} \supset p_{2}\right)\right)\right) \wedge\left(\left(p_{5} \supset\left(p_{5} \supset p_{4}\right)\right)\right) \wedge\left(\left(p_{6} \supset\right.\right.$ $\left.\left.\left(p_{6} \supset p_{5}\right)\right)\right) \wedge\left(\left(p_{7} \supset\left(p_{7} \supset p_{6}\right)\right)\right) \wedge\left(\left(p_{8} \supset\left(p_{8} \supset p_{7}\right)\right)\right) \wedge\left(\left(p_{1} \supset\left(p_{1} \supset p_{8}\right)\right)\right) \supset$ $\left(p_{3}\right)$

41. $((\square D \equiv(C \supset \diamond A)) \equiv \diamond R) \supset(\diamond R \equiv((C \supset \diamond A)) \equiv \square D)$

42. $(((\square A \vee \diamond B) \supset(\square C \wedge \square \square D) \supset(\diamond B \wedge \diamond \diamond C) \supset \diamond E) \vee \square F) \supset(\diamond F \vee((\square A \vee$ $\diamond B) \supset(\square C \wedge \square \square D) \supset(\diamond B \wedge \diamond \diamond C) \supset \diamond E))$

43. $\left(\left(p_{1} \vee p_{2}\right)\right) \wedge\left(\left(p_{3} \vee p_{4}\right)\right) \wedge\left(\left(p_{5} \vee p_{6}\right)\right) \supset\left(\left(\left(p_{1} \wedge p_{3}\right) \vee\left(\left(p_{1} \wedge p_{5}\right) \vee\left(\left(p_{3} \wedge p_{5}\right) \vee\right.\right.\right.\right.$ $\left.\left.\left.\left.\left(\left(p_{2} \wedge p_{4}\right) \vee\left(\left(p_{2} \wedge p_{6}\right) \vee\left(p_{4} \wedge p_{6}\right)\right)\right)\right)\right)\right)\right)$

44. $\left(\square\left(p_{1} \vee p_{2}\right)\right) \wedge\left(\square\left(\square p_{3} \vee \square p_{4}\right)\right) \wedge\left(\left(\square p_{5} \vee \square p_{6}\right)\right) \supset\left(\left(\left(\diamond p_{1} \wedge \diamond p_{3}\right) \vee\left(\left(\diamond p_{1} \wedge\right.\right.\right.\right.$ $\left.\left.\left.\left.p_{5}\right) \vee\left(\left(\diamond p_{3} \wedge p_{5}\right) \vee\left(\left(\diamond p_{2} \wedge \diamond p_{4}\right) \vee\left(\left(\diamond p_{2} \wedge p_{6}\right) \vee\left(\diamond p_{4} \wedge p_{6}\right)\right)\right)\right)\right)\right)\right)$

45. $\left(\left(\left(\left(p_{1} \wedge\left(p_{2} \wedge\left(p_{3} \wedge\left(p_{4} \wedge\left(p_{5} \wedge p_{6}\right)\right)\right)\right)\right) \vee\left(\left(p_{1} \supset p_{7}\right) \vee\left(\left(p_{2} \supset p_{7}\right) \vee\left(\left(p_{3} \supset\right.\right.\right.\right.\right.\right.\right.$ $\left.\left.\left.\left.\left.\left.\left.p_{7}\right) \vee\left(\left(p_{4} \supset p_{7}\right) \vee\left(\left(p_{5} \supset p_{7}\right) \vee\left(p_{6} \supset p_{7}\right)\right)\right)\right)\right)\right)\right) \supset p_{7}\right)\right) \supset\left(p_{7}\right)$

46. $\left(\left(\left(\left(\diamond p_{1} \wedge\left(\square p_{2} \wedge\left(\diamond p_{3} \wedge\left(\square p_{4} \wedge \diamond p_{5}\right)\right)\right)\right) \vee\left(\left(\diamond p_{1} \supset p_{6}\right) \vee\left(\left(\square p_{2} \supset p_{6}\right) \vee\left(\left(\diamond p_{3} \supset\right.\right.\right.\right.\right.\right.\right.$ $\left.\left.\left.\left.\left.\left.\left.p_{6}\right) \vee\left(\left(\square p_{4} \supset p_{6}\right) \vee\left(\diamond p_{5} \supset p_{6}\right)\right)\right)\right)\right)\right) \supset p_{6}\right)\right) \supset\left(p_{6}\right)$

47. $\left(p_{1}\right) \wedge\left(\left(p_{2} \supset\left(\left(p_{3} \vee p_{4}\right) \vee p_{3}\right)\right)\right) \wedge\left(\left(p_{5} \supset\left(\left(p_{2} \vee p_{6}\right) \vee p_{2}\right)\right)\right) \wedge\left(\left(\left(p_{5} \vee p_{7}\right) \vee p_{5}\right)\right) \supset$ $\left(\left(p_{7} \vee\left(\left(p_{5} \wedge p_{6}\right) \vee\left(\left(p_{2} \wedge p_{4}\right) \vee\left(p_{3} \wedge p_{1}\right)\right)\right)\right)\right)$

48. $\left(\left(\left(\square \square p_{1}\right) \supset\left(\left(\neg\left(\neg \diamond \diamond p_{1}\right)\right)\right)\right) \supset\left(\left(p_{1}\right) \supset\left(\left(\neg\left(\neg \diamond p_{1}\right)\right)\right)\right)\right) \supset\left(\left(\left(\square \square p_{1}\right) \supset((\neg(\neg\right.\right.$ $\left.\left.\left.\left.\diamond \diamond p_{1}\right)\right)\right) \supset\left(\left(p_{1}\right) \supset\left(\left(\neg\left(\neg \diamond p_{1}\right)\right)\right)\right)\right)$

49. $\left(\left(\left(\neg \diamond p_{1}\right) \vee\left(\neg \diamond p_{2}\right)\right)\right) \supset\left(\left(\left(\diamond \neg p_{2}\right) \vee\left(\diamond \neg p_{1}\right)\right)\right)$

50. $\left(p_{1}\right) \wedge \square\left(\left(p_{2} \supset\left(\left(\square p_{3} \vee \square p_{4}\right) \vee \square p_{3}\right)\right)\right) \wedge \square\left(\left(p_{5} \supset\left(\left(p_{2} \vee p_{6}\right) \vee p_{2}\right)\right)\right) \wedge\left(\left(\left(p_{5} \vee\right.\right.\right.$ $\left.\left.\left.p_{7}\right) \vee p_{5}\right)\right) \supset \diamond\left(\left(p_{7} \vee\left(\left(\diamond p_{5} \wedge p_{6}\right) \vee\left(\left(p_{2} \wedge p_{4}\right) \vee\left(p_{3} \wedge p_{1}\right)\right)\right)\right)\right)$ 